\title{
Nonlinear Supersymmetric Quantum Mechanics: concepts and realizations
}

\author{
A A Andrianov†§ and M V Ioffe $\dagger$ \\ $\dagger$ V A Fock Department of Theoretical Physics, Saint-Petersburg State University, \\ 198504 Sankt-Petersburg, Russia \\ $\S$ Departament d'Estructura i Constituents de la Matèria and Institut de Ciències del \\ Cosmos (ICCUB), Universitat de Barcelona, 08028 Barcelona, Spain \\ E-mail: andrianov@icc.ub.edu; m.ioffe@pobox.spbu.ru
}

\begin{abstract}
Nonlinear SUSY approach to spectral problems in Quantum Mechanics is reviewed. Its building from the chains (ladders) of linear SUSY systems is outlined and different one-dimensional and two-dimensional realizations are described. For various two-dimensional models it is elaborated how Nonlinear SUSY provides two new methods of supersymmetric separation of variables. In the framework of these methods partial and/or complete solution of some two-dimensional models becomes possible. The full classification of ladder-reducible and irreducible chains of SUSY algebras in one-dimensional QM is given. Emergence of hidden symmetries and spectrum generating algebras is elucidated in the context of Nonlinear SUSY in one-dimensional stationary and non-stationary as well as in two-dimensional QM.
\end{abstract}

Submitted to: Journal of Physics A: Mathematical and Theoretical

PACS numbers: 03.65.Ca,03.65.Fd,11.30.Pb 


\section{Contents}

1 Introduction 4

2 SUSY QM in arbitrary space dimension 9

2.1 Two-dimensional SUSY QM with first-order supercharges . . . . . . . . . 99

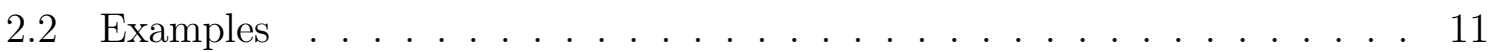

2.3 SUSY QM in arbitrary dimensionality of space as $(0+1)$-dimensional Quantum Field Theory . . . . . . . . . . . . . . . 13

2.4 The structure of super-Hamiltonian in SUSY QM for arbitrary dimensionality of space . . . . . . . . . . . . . 15

2.5 The particular case of $d=3$ : nucleon-nucleon interaction . . . . . . 17

3 Polynomial SUSY in $\mathrm{d}=\mathbf{1} \quad$

3.1 Supercharges of higher order in derivatives from ladder construction . . . 18

3.2 Polynomial SUSY QM from a ladder of linear SUSY systems . . . . . . 21

3.3 Generation of Polynomial SUSY QM by ladder: irreducible SUSY blocks of type I, II, and III . . . . . . . . . . . . . . . . . . . . . . . . . . 22

3.4 Minimization of SUSY algebra for a given Hamiltonian and emergence of irreducible blocks of type II and III . . . . . . . . . . . . . . . . . 24

4 Rigorous results on structure and (ir)reducibility of Polynomial SUSY 27

4.1 Basic theorems on the structure of QM with a nonlinear SUSY . . . . . 27

4.2 Classification of really (ir)reducible SUSY transformations . . . . . . . . 29

$5 \quad$ Hidden symmetry in One-dimensional SUSY QM 31

5.1 Simplest SUSY with Hidden symmetry . . . . . . . . . . . . . . . 31

5.2 General case: one-dim SUSY algebra with Hidden symmetry . . . . . . . 33

6 Shape invariance for SUSY related potentials 36

6.1 Shape invariance in linear SUSY . . . . . . . . . . . . . . 36

6.2 Intertwining with shift and higher order shape-invariance . . . . . . . . . 37

6.3 Intertwining with shift and second order shape-invariance . . . . . . . . . 38

6.4 Intertwining with shift and third order shape-invariance . . . . . . . . . . 40

6.5 Spectrum patterns for third order shape-invariance . . . . . . . . . . . . 41

7 Nonlinear SUSY for non-stationary Schrödinger equations 4

7.1 Linear SUSY and hidden symmetry . . . . . . . . . . . . . . . . . 43

7.2 Second-order intertwining for stationary potentials: spectrum generating algebra . . . . . . . . . . . . . . . . . . 45

8 Polynomial SUSY QM in $d=2 \quad 48$

8.1 Supercharges of second order in derivatives: generalities . . . . . . . . . . 48

8.2 Hyperbolic (Lorentz) metrics . . . . . . . . . . . . . . . . . . 50 
8.2.1 Supercharges with twisted reducibility. . . . . . . . . . . . 50

8.2 .2 Irreducible supercharges. . . . . . . . . . . . . . . . . . . . . . . . . . . .

8.3 Integrability . . . . . . . . . . . . . . . . . 54

9 SUSY separation of variables for $d=2 \quad \mathbf{5 5}$

9.1 SUSY-separation of variables I: QES models . . . . . . . . . . . . . . . . . . . .

9.2 SUSY-separation of variables II: Exact solvability. . . . . . . . . . . . . 59

10 Perspectives, some applications and missing points $\quad 62$

11 References $\quad 64$ 


\section{Introduction}

The concept of Supersymmetric Quantum Mechanics (SUSY QM) embodies an algebraic form of transformations of (complete or partial) spectral equivalence between different dynamical systems and in this sense it gives the algebraic tools for spectral design of new quantum systems from a given set with controllable energy spectra. At present, there is a number of reviews [1]-[18] devoted to development and various applications, mostly of the linear SUSY QM. The very isospectral transformations realizing SUSY represent the Darboux (Darboux-Moutard) transformations [19]-29], which are known in the theory of ordinary differential equations for a long time. According to [21] these transformations must be referred as Euler-Imshenetsky-DarbouxMoutard ones having their origin in the paper by Leonard Euler [23]. We thank our referee for drawing our attention to this chain of old papers.

The Supersymmetric Quantum Mechanics in its form was formulated for onedimensional space by E.Witten [30] about thirty years ago(see also [31 - [33]). This approach has two predecessors: Factorization Method [34] in Quantum Mechanics and Darboux transformation [19]-28] for the Sturm-Liouville equation in Mathematics.

The first one was proposed by E.Schrödinger in 1940-ties [34] (see the excellent detailed review [35]), and its basic relation is the representation of Hamiltonian in a factorized form,

$$
h^{(0)}(x)=-\partial^{2}+V^{(0)}(x)=q^{+} q^{-},
$$

where operators $q^{ \pm}$are,

$$
q^{ \pm}=\mp \partial+(\partial W)(x) ; \quad q^{+}=\left(q^{-}\right)^{\dagger} \quad \partial \equiv \frac{d}{d x} .
$$

The factorization (11) is in general possible for an arbitrary potential $V(x)$ (see details below). The main idea of Factorization Method is to consider together with the Hamiltonian (11) the partner Hamiltonian, also of Schrödinger form,

$$
h^{(1)}(x)=-\partial^{2}+V^{(1)}(x)=q^{-} q^{+},
$$

with the same operators $q^{ \pm}$. By construction, these Hamiltonians satisfy the intertwining relations,

$$
h^{(0)} q^{+}=q^{+} h^{(1)} ; \quad q^{-} h^{(0)}=h^{(1)} q^{-},
$$

which play the key role in the method under discussion. Just the intertwining relations (4) allow to obtain the simple relations between wave functions $\Psi_{n}^{(0)}$ and $\Psi_{n}^{(1)}$ of $h^{(0)}$ and $h^{(1)}$, correspondingly. Indeed, according to (4) an arbitrary wave function $\Psi_{n}^{(0)}(x)$ of $h^{(0)}$ gives the wave function $\Psi_{n}^{(1)}$ of $h^{(1)}$, and vice versa,

$$
q^{-} \Psi_{n}^{(0)} \propto \Psi_{n}^{(1)} ; \quad q^{+} \Psi_{n}^{(1)} \propto \Psi_{n}^{(0)} .
$$

The energy eigenvalues for $\Psi_{n}^{(0)}$ and $\Psi_{n}^{(1)}$ are the same, $E_{n}^{(0)}=E_{n}^{(1)}$. There are three options [36] for the spectra of $h^{(0)}$ and $h^{(1)}$ :

- spectra coincide completely if neither $q^{+}$, no $q^{-}$have normalizable zero modes; 
- spectra almost coincide: the spectrum of $h^{(0)}$ has one bound state less, if the normalizable zero mode of $q^{+}$exists;

- spectra almost coincide: the spectrum of $h^{(1)}$ has one bound state less, if the normalizable zero mode of $q^{-}$exists.

This is an essence of the Spectral Design method based on the super-Hamiltonian $H$ with two non-singular real potentials. The supersymmetry results in isospectral relations between the super-Hamiltonian components $h^{(0)}$ and $h^{(1)}$ whereas two nonsingular real potentials are different. Each of the operators $q^{ \pm}$may have one zero mode $\exp ( \pm W(x))$, and their normalizability properties depend on the behavior of function $W(x)$ : the Hamiltonians $h^{(0)}$ and $h^{(1)}$ are [36]- 43] either isospectral or almost isospectral (up to one bound state).

The connection between a given potential $V^{(0)}(x)$ and a function $W(x)$ provides a basis for the link of Factorization Method with Darboux transformation [19] and represents the analytic tool of Spectral Design. The substitution of (2) into (11), (3) results in:

$$
\begin{aligned}
& V^{(0)}(x)=(\partial W)^{2}(x)-\left(\partial^{2} W\right)(x) ; \\
& V^{(1)}(x)=(\partial W)^{2}(x)+\left(\partial^{2} W\right)(x) .
\end{aligned}
$$

In terms of the theory of ordinary second-order differential Sturm-Liouville equations (adapted to the Schrödinger form),

$$
-\Psi^{(0) \prime \prime}(x)+V^{(0)}(x) \Psi^{(0)}(x)=E^{(0)} \Psi^{(0)}(x),
$$

the Darboux transformation can be formulated as follows. If one knows its particular solution $\psi^{(0)}(x) \equiv \exp (-W(x))$ for the value $E^{(0)}=0$, the Eq.(6) is satisfied, and an arbitrary solution $\Psi^{(0)}\left(x ; E^{(0)}\right)$ of (8) can be transformed (Darboux transformation) into a solution of the transformed equation of the same form and with the same value of parameter $E^{(0)}$,

$$
-\Psi^{(1) \prime \prime}(x)+V^{(1)}(x) \Psi^{(1)}(x)=E^{(0)} \Psi^{(1)}(x) .
$$

The initial and transformed solutions are connected,

$$
\begin{aligned}
& \Psi^{(1)}\left(x ; E^{(0)}\right) \propto(\partial+(\partial W)(x)) \Psi^{(0)}\left(x ; E^{(0)}\right) ; \\
& \Psi^{(0)}\left(x ; E^{(0)}\right) \propto(-\partial+(\partial W)(x)) \Psi^{(1)}\left(x ; E^{(0)}\right) .
\end{aligned}
$$

It is clear that one obtains a one-to-one correspondence between solutions of (8) and (9), but up to zero modes of operators $(\mp \partial+(\partial W)(x))$.

The nonlinear first-order differential equation (6) (Riccati[44 equation) can be solved analytically for a restricted class of potentials $V^{(0)}(x)$ only. The class of analytically solvable Riccati equations corresponds to the so called exactly solvable models in one-dimensional Quantum Mechanics, and latter ones are related to the so called shape-invariant [45] potentials. 
The above construction can be easily presented in a supersymmetric form. Indeed, the Hamiltonians $h^{(0)}, h^{(1)}$ and intertwining operators $q^{ \pm}$can be considered as elements of matrix $2 \times 2$ operators - super-Hamiltonian and supercharges,

$H=\left(\begin{array}{cc}h^{(0)} & 0 \\ 0 & h^{(1)}\end{array}\right) ; \quad Q^{+}=\left(\begin{array}{cc}0 & 0 \\ q^{-} & 0\end{array}\right) ; \quad Q^{-}=\left(Q^{+}\right)^{\dagger}=\left(\begin{array}{cc}0 & q^{+} \\ 0 & 0\end{array}\right)$,

which obey the (anti)commutation relations - the simplest realization of supersymmetric algebra,

$$
\left[H, Q^{ \pm}\right]=0 ; \quad\left\{Q^{+}, Q^{-}\right\}=H ; \quad\left(Q^{ \pm}\right)^{2}=0 .
$$

The relations (12) of the superalgebra express in the compact form the factorization (11), (3) and the intertwining relations (44), while the nilpotency of supercharges $Q^{ \pm}$ follows directly from their matrix structure. By analogy with the Supersymmetric Quantum Field Theory, the component $h^{(0)}$ of $H$ is called the bosonic component, and $h^{(1)}$ fermionic one. Correspondingly, their eigenstates form bosonic and fermionic sectors, although this terminology has no physical sense in the present non-relativistic context. Nevertheless, the nilpotency of $Q^{ \pm}$can be associated with the fermion property - an analog of the Pauli principle.

Thus in the simplest cases (linear SUSY algebras), intertwining of two differential operators (for instance, Hamiltonians of one-dimensional quantum systems) by means of Darboux operators of first order in derivatives entails their factorization into differential multipliers which are formed by the same Darboux operators. However, in general, both interrelation between pairs of dynamical operators ("Hamiltonians") with (almost) equivalent spectra and structure of operators which generate the spectral equivalence are not that simple [46] - 67]. In particular, the closing of a Nonlinear SUSY algebra often produces a hidden symmetry generator which helps to partial solvability of spectral problem for Schrödinger Hamiltonians. This interrelation in the one- and twodimensional QM is within the scope of the present review. In particular, we survey well motivated solutions to the following problems:

- what is the correct self-consistent way to generalize the main relations of onedimensional SUSY Quantum Mechanics, including factorization and intertwining relations, to the case of multidimensional space?

- in what cases the higher-order Darboux-Crum transformations can be constructed with the help of a sequence of intertwining transformations of lower order SUSY which relate a chain of (almost) isospectral intermediate Hamiltonians with real nonsingular potentials?

- what are elementary blocks for a nonsingular factorization of intertwining operators?

- in what way the irreducibility of elementary blocks of isospectral transformations reveals itself in the SUSY algebra and in the structure of kernels of those transformations? 
- are there more complicated SUSY algebras in one dimensional QM which accept hidden symmetry generators ("central charges")?

- what is the relationship between shape-invariance and spectrum generating algebras for SUSY QM systems?

- how SUSY for non-stationary Schrödinger operators produces hidden symmetries and spectrum generating algebras?

- is it possible to find pairs of (almost) isospectral scalar two-dimensional Hamiltonians intertwined by higher order supercharges, i.e. to avoid the matrix components of super-Hamiltonian by means of using Nonlinear SUSY algebra?

- does the Nonlinear two-dimensional SUSY Quantum Mechanics give the constructive way to obtain new exactly solvable and/or quasi-exactly solvable models not amenable to the conventional separation of variables?

The structure of the paper is as follows. After a short reminder of notation and basic definitions of SUSY theory of isospectral transformations given above in the Introduction, the generalizations of linear SUSY QM for arbitrary space dimensions [36], [68] are considered in Section 2. All of them can be called as direct generalizations since they are based on the supercharge operators of first order in derivatives. In particular, Subsection 2.1 includes a detailed description of two-dimensional generalization [36] in the form similar to the Factorization Method of E.Schrödinger [34, [35], and Subsection 2.2 contains two illustrative examples: Pauli equation for fermion motion on the plane with external magnetic field [69] - [71] and supersymmetric extension of two-dimensional attractive Coulomb potential [36]. In Subsection 2.3, the well developed formalism of Supersymmetric Quantum Field Theory is used to present the direct (first-order) generalization [68] of Witten's one-dimensional SUSY Quantum Mechanics onto the space with arbitrary number $d$ of dimensions. The main element of construction is the $d$-component real scalar superfield depending both on time and pair of Grassmann variables. This multidimensional generalization allows also the alternative interpretation as a model of $d$ interacting particles on a line [18]. In Subsection 2.4 the structure of obtained super-Hamiltonian is analyzed [68] in the Hilbert space which is a direct sum of subspaces with fixed fermion numbers. In this basis, the super-Hamiltonian has the block-diagonal form with matrix blocks of different matrix dimensionality. The supersymmetric intertwining relations between different matrix components of the super-Hamiltonian are derived providing their isospectrality. Subsection 2.5 gives a brief description of the illustrative example [72]: $d=3$ SUSY Quantum Mechanics with specific Yukawa-like superpotential provides relations between different sectors of nucleon-nucleon and nucleon-antinucleon interactions.

Section 3 is devoted to building of the Polynomial SUSY algebra for $d=1$ with the help of dressing chain or ladder construction [46]. It is elucidated how they entail the SUSY algebra representation by differential intertwining operators of higher-order in derivatives. For algebraic formulation its key ingredient is given by SUSY Hamiltonian projections on the formal zero-mode space (the kernel) of supercharge operators which 
are finite-dimensional constant matrices [65, 73]. However the basic elements of spectral design in Polynomial SUSY consist not only of intermediate isospectral transformations of first order in derivative (of linear SUSY's) but also of the transformations of second order in derivative [47, 54, 74]. Three irreducible classes of binary SUSY algebras are revealed and it is shown how they serve to complete the tool kit for spectral design. In particular, they may help to add or remove one or two adjacent levels for excited states. Thus the classification of irreducible (almost) isospectral transformations and related SUSY algebras is outlined. It was described first in [14, then systematically in [75, 76]) and the exhaustive fine classification of irreducible operators was completed in [77]. In connection to this classification the important problem of a possible redundancy in supercharges which can be eliminated without any changes in the super-Hamiltonian is analyzed in Subsection 3.4. With the help of a (redundant) multiplication of supercharge by a polynomial of super-Hamiltonian it is elucidated that certain irreducible secondorder supercharges can be embedded into a higher-order SUSY which in turn is decomposable in a chain of linear SUSY's. In Section 4 we formulate basic theorems which give full description of the structure of polynomial one-dimensional SUSY algebras and on minimization of this algebra up to its essential part (proofs of these theorems can be found in [73, 75, 76, 77]). Apart from the above mentioned trivial ways to generate several SUSY algebras for the same super-Hamiltonian there exist possibilities to obtain two non-minimizable SUSY algebras with supercharges of even and odd order in derivatives [73, 78]. Their combination forms a non-trivial hidden-symmetry operator which cannot be expressed as a polynomial of the super-Hamiltonian. Such systems and algebras are described in Section 5. They are governed by (reflectionless) potentials of a special form arising also as soliton solutions of some non-linear equations [28, 29]. The related SUSY algebra can be qualified as $\mathcal{N}=2$ superalgebra with central charge made of hidden symmetry operators.

In Sect.6 the concept of shape invariance is formulated for $d=1$ polynomial SUSY algebras with the help of intertwining with shift [78]. The arising spectrum generating algebras represent intrinsic features of shape invariance and they can be considered as deformations of hidden symmetries studied in Sect.5 . It is elucidated on and exemplified for the second- and third-order SUSY shape invariance. The relations with deformed Heisenberg algebras [11, 58, 59] are described as well. In the case of third-order SUSY its remarkable connection to the Painleve-IV equations [79] is demonstrated (Sect. 6.4) and the typical patterns of energy spectra are listed. The latter connection has been in focus of recent investigations [80]-86].

Section 7 is devoted to extension of spectral design methods on nonstationary Schrödinger equations [87]. We follow the scheme of SUSY intertwining relations and show that addition of time dependence leads to certain restrictions on potentials which are assembled in a super-Hamiltonian. In all cases the closure of the SUSY algebra reveals a hidden symmetry which becomes a typical rather than exceptional feature for non-stationary Hamiltonians. For linear SUSY (Sect. 7.1)this hidden symmetry entails simply full separation of variables. For the second-order SUSY (Sect. 7.2) one 
discovers the spectrum generating algebra previously found for the third-order shape invariance and this algebra is completely encoded in time-dependent hidden symmetry operators. Thus hidden symmetries for nonstationary Schrödinger super-Hamiltonians give the universal framework for both stationary hidden symmetries and for spectrum generating algebras of shape invariant SUSY systems.

In Section 8, the idea of one-dimensional Polynomial SUSY QM is generalized onto two-dimensional case providing isospectrality of scalar Schrödinger Hamiltonians without any intermediate matrix components [88] - [105]. Subsection 8.1 gives the basic formulas [88] - 91] of this approach for arbitrary form of highest (second-order) terms in supercharge. The most promising case of Lorentz (hyperbolic) form of second-order terms is studied in Subsection 8.2 with special attention to reducible (with twist) and irreducible supercharges. The fundamental difference of two-dimensional Polynomial SUSY QM is that both SUSY-partner Hamiltonians are completely integrable: they commute with symmetry operators which are of fourth order in momenta (Subsection 8.3). Other examples of relation between nonlinear supersymmetry and quasi-exact solvability can be found in [106]. Section 9 is devoted to two new methods [13, [16] of solution of two-dimensional Schrödinger equations which are not amenable to conventional method of separation of variables. Both new methods are based on the isospectrality of scalar Hamiltonians established in the previous Section and on the property of shape invariance (Section 6). These methods can be considered as supersymmetric separation of variables. The first of them (Subsection 9.1) provides the quasi-exact solvability of several two-dimensional models, i.e. it allows to find analytically a part of spectrum and corresponding wave functions. The second one (Subsection 9.2) gives the exact analytical solution of the models but it works only for particular values of parameters of the models. Both methods can be applied to several potentials not amenable to standard separation of variables, and here they are illustrated by solution of two-dimensional generalization of Morse model. In Section 10, we list some related problems of SUSY QM which are outside the scope of the present review, and we mention some related open problems and perspectives for future study.

\section{SUSY QM in arbitrary space dimension}

\subsection{Two-dimensional SUSY QM with first-order supercharges}

The generalization of one-dimensional SUSY QM onto higher space dimensionality $d \geq 2$ in the Schrödinger equation is of great interest both from the conceptual and practical point of view. The forthright attempt to preserve the one-dimensional formulas given above but with superpotentials depending on multidimensional coordinate vector $\vec{x}=\left(x_{1}, x_{2}, \ldots, x_{d}\right)$ leads to potentials which are amenable to standard separation of variables (see details in [107]). In what follows such generalizations will not be considered since the corresponding Schrödinger equation is reduced to the simpler problems of lower dimensionalities. 
A more advanced generalization of one-dimensional SUSY QM onto multidimensional case was made in the framework of Factorization Method in [36] (see also [108] and [109]). The main idea is in involving vector intertwining operators $q_{l}(\vec{x}) ; l=1,2, \ldots, d$, which are still differential operators of first order in derivatives,

$$
\left.q_{l}^{ \pm}(\vec{x})=\mp \partial_{l}+\left(\partial_{l} W\right)(\vec{x})\right) ; \quad \vec{x}=\left(x_{1}, x_{2}, \ldots, x_{d}\right) ; \quad \partial_{l} \equiv \frac{\partial}{\partial x_{l}} .
$$

In terms of these operators, the initial Hamiltonian $h^{(0)}(\vec{x})$ can be quasifactorized,

$$
h^{(0)}(\vec{x})=-\triangle^{(2)}+V^{(0)}(\vec{x})=q_{l}^{+} q_{l}^{-} ; \quad \triangle^{(2)} \equiv \partial_{l} \partial_{l},
$$

where the sum over repeated index $l$ is implied. In the framework of this generalization, which can be named as "direct generalization", the Schrödinger operator $h^{(0)}$ is related to the chain of $d$ other Hamiltonians of Schrödinger form, but $(d-1)$ of them with matrix potentials (see Subsection 2.5 below).

Now we shall describe this construction [36 in the simplest - two-dimensional $d=2$ - case, where the chain of Hamiltonians includes three species, the original $h^{(0)}$ and two others, $h_{i k}^{(1)}$ and $h^{(2)}$, both of the Schödinger form as well. The first of them has $2 \times 2$ matrix potential $V_{i k}^{(1)}(\vec{x})$, while the second one has a scalar potential $V^{(2)}(\vec{x})$. These partner Hamiltonians (superpartners) are expressible in a quasifactorized form as well,

$h_{i k}^{(1)}=q_{i}^{-} q_{k}^{+}+p_{i}^{-} p_{k}^{+}=-\delta_{i k} \partial_{l}^{2}+\delta_{i k}\left(\left(\partial_{l} W\right)^{2}(\vec{x})-\left(\partial_{l}^{2} W\right)(\vec{x})\right)+2\left(\partial_{i} \partial_{k} W\right)(\vec{x}) ;$

$h^{(2)}=p_{l}^{+} p_{l}^{-}=-\partial_{l}^{2}+V^{(2)}(\vec{x})=-\partial_{l}^{2}+\left(\partial_{l} W\right)^{2}(\vec{x})+\left(\partial_{l}^{2} W\right)(\vec{x}) ;$

The operators $q_{l}^{ \pm}$were defined in (13), and the new vector operators $p_{l}^{ \pm}$are also of first order in derivatives,

$$
p_{l}^{ \pm} \equiv \epsilon_{l k} q_{k}^{\mp},
$$

where $\epsilon_{l k}$ is a standard antisymmetric tensor with $\epsilon_{12}=+1$.

The chain of two-dimensional Hamiltonians $h^{(0)}, h_{i k}^{(1)}, h^{(2)}$ is analogous to the one-dimensional partnership $h^{(0)}, h^{(1)}$, since its components are also related by the intertwining relations with operators $q_{l}^{ \pm}, p_{l}^{ \pm}$playing the role of intertwining operators,

$$
\begin{array}{ll}
h^{(0)} q_{i}^{+}=q_{k}^{+} h_{k i}^{(1)} ; & h_{i k}^{(1)} q_{k}^{-}=q_{i}^{-} h^{(0)} ; \\
h_{i k}^{(1)} p_{k}^{-}=p_{i}^{-} h^{(2)} ; & p_{k}^{+} h_{k i}^{(1)}=h^{(2)} p_{i}^{+} .
\end{array}
$$

By definition, the intertwining operators $q_{l}^{ \pm}, p_{l}^{\mp}$, and, correspondingly, two terms in the definition (15) of $h_{i k}^{(1)}$ are mutually orthogonal, $q_{l}^{ \pm} p_{l}^{\mp}=0$. These two terms in $h_{i k}^{(1)}$ were necessary to obtain the Hamiltonian of the Schrödinger form, and the orthogonality is important to provide the intertwining relations (18), (19).

The energy spectra of $h^{(0)}$ and $h^{(2)}$ are, in general, non-overlapping, but the intertwining relations (18), (19) provide the equivalence of energy spectra between a pair of two scalar Hamiltonians $h^{(0)}, h^{(2)}$ and $2 \times 2$ matrix Hamiltonian $h_{i k}^{(1)}$. The "equivalence" means coincidence of their spectra up to zero modes of operators $q_{l}^{ \pm}, p_{l}^{ \pm}$. Thus, the supersymmetry (supersymmetric transformation) allows to reduce the solution of spectral problem for the matrix Hamiltonian $h_{i k}^{(1)}$ to solutions of the couple of scalar 
spectral problems $h^{(0)}, h^{(2)}$ realizing the SUSY diagonalization method. Due to the intertwining relations, the vector wave functions of matrix Hamiltonian $h_{i k}^{(1)}$ are also connected (up to a normalization factor) with the scalar wave functions of scalar Hamiltonians $h^{(0)}, h^{(2)}$,

$$
\begin{aligned}
& \Psi_{i}^{(1)}(\vec{x} ; E)=q_{i}^{-} \Psi^{(0)}(\vec{x} ; E) ; \quad i=1,2 ; \quad \Psi^{(0)}(\vec{x} ; E)=q_{i}^{+} \Psi_{i}^{(1)}(\vec{x} ; E) \\
& \Psi_{i}^{(1)}(\vec{x} ; E)=p_{i}^{-} \Psi^{(2)}(\vec{x} ; E) ; \quad \Psi^{(2)}(\vec{x} ; E)=p_{i}^{+} \Psi_{i}^{(1)}(\vec{x} ; E) .
\end{aligned}
$$

The two-dimensional generalization (14), (15), (16), (18), (19) of Factorization Method can be also formulated compactly [68] in terms of exactly the same superalgebra (12). Indeed, three Hamiltonians $h^{(0)}, h_{i k}^{(1)}, h^{(2)}$ are combined in the super-Hamiltonian $H$, and intertwining operators $q_{l}^{ \pm}, p_{l}^{ \pm}$- in the supercharges $Q^{ \pm}$, correspondingly,

$$
H=\left(\begin{array}{ccc}
h^{(0)} & 0 & 0 \\
0 & h_{i k}^{(1)} & 0 \\
0 & 0 & h^{(2)}
\end{array}\right) ; \quad Q^{+}=\left(Q^{-}\right)^{\dagger}=\left(\begin{array}{cccc}
0 & 0 & 0 & 0 \\
q_{1}^{-} & 0 & 0 & 0 \\
q_{2}^{-} & 0 & 0 & 0 \\
0 & p_{1}^{+} & p_{2}^{+} & 0
\end{array}\right) \text {, }
$$

all matrices are $4 \times 4$. The components $h^{(0)}, h^{(2)}$ are bosonic, and $h_{i k}^{(1)}-$ fermionic component of the super-Hamiltonian.

A simple rearrangement of this representation gives an idea of SUSY diagonalization of matrix Hamiltonians in terms of the conventional SUSY algebra (12),

$$
\begin{aligned}
& \widetilde{H}=\left(\begin{array}{cc}
\tilde{h}^{(0)} & 0 \\
0 & \tilde{h}^{(1)}
\end{array}\right) ; \quad \tilde{h}^{(0)}=\left(\begin{array}{cc}
h^{(0)} & 0 \\
0 & h^{(2)}
\end{array}\right) ; \quad \tilde{h}^{(1)}=\left(h_{i k}^{(1)}\right) ; \\
& \widetilde{Q}^{+}=\left(\widetilde{Q}^{-}\right)^{\dagger}=\left(\begin{array}{cc}
0 & 0 \\
\tilde{q}^{-} & 0
\end{array}\right) ; \quad \tilde{q}^{-}=\left(\tilde{q}^{+}\right)^{\dagger}=\left(\begin{array}{cc}
q_{1}^{+} & q_{2}^{+} \\
p_{1}^{+} & p_{2}^{+}
\end{array}\right) .
\end{aligned}
$$

\subsection{Examples}

The Schrödinger operators with matrix potentials are not something very exotic in Quantum Mechanics. In particular, the described two-dimensional generalization of SUSY QM was successfully used [69 - [71] to investigate the spectra of stationary Pauli operator for fermion in external nonhomogeneous electromagnetic fields.

1. The Pauli Hamiltonian for nonrelativistic spin $1 / 2$ particle in three-dimensional space is written as follows,

$$
H_{P}=\left(i \partial_{a}+e A_{a}\right)^{2}-\mu \sigma_{a} B_{a}+U,
$$

where $a=1,2,3, e$ and $\mu$ are the electric charge and magnetic momentum of the particle, $\left\{A_{a}(\vec{x})\right\}$ is the vector part of electromagnetic potential, $\vec{B}(\vec{x})=\operatorname{rot} \vec{A}(\vec{x})$ is the corresponding magnetic field (in general, nonhomogeneous), $U(\vec{x})$ is the scalar potential (which may include the electrostatic term $e A_{0}(\vec{x})$ ), and $\sigma_{a}$ are the standard Pauli matrices. Restricting the problem to the case of external potentials which do not depend on one of coordinates (on $x_{2}$ below), the Pauli Hamiltonian (23), being $2 \times 2$ matrix 
operator, can be identified with the matrix component $h_{i k}^{(1)} ;(i, k=1,2)$ of the superHamiltonian in the previous Subsection. Indeed, the fermionic (two-component) wave function has to be factorized into the trivial plane wave along $x_{2}$ with some momentum $k$ and nontrivial two-dimensional wave function,

$$
\Psi_{P}(\vec{x})=\exp \left(i k x_{2}\right) \Psi\left(x_{1}, x_{3}\right) .
$$

In the subspace of such functions, Pauli operator can be rewritten as,

$$
H_{P}\left(x_{1}, x_{3}\right)=-\left(\partial_{l}+i e A_{l}\right)^{2}+U+\left(k+e A_{2}\right)^{2}-\mu \sigma_{a} B_{a} ; \quad l=1,3 ; \quad A_{l}=A_{l}\left(x_{1}, x_{3}\right) .
$$

Comparing (25) and (15), one concludes that their identification (up to replacing $\left.\left(x_{1}, x_{3}\right) \leftrightarrow\left(x_{1}, x_{2}\right)\right)$ is possible if external fields in (25) are expressed in terms of the only function $W\left(x_{1}, x_{3}\right)$,

$$
-\mu \vec{B}=\left(2\left(\partial_{1} \partial_{3} W\right)\left(x_{1}, x_{3}\right), 0,\left(\left(\partial_{1}^{2}-\partial_{3}^{2}\right) W\right)\left(x_{1}, x_{3}\right)\right) .
$$

Let for simplicity the external sources of magnetic field are absent,

$$
\operatorname{rot} \vec{B}=\operatorname{div} \vec{B}=0
$$

Then, the function $W$ is a polynomial of fourth order, and magnetic field is of the form,

$$
-\mu \vec{B}=\left(\left(2 a x_{1} x_{3}+c x_{1}+b x_{3}+g\right), 0,\left(a x_{1}^{2}-a x_{3}^{2}+b x_{1}-c x_{3}+d\right)\right),
$$

with arbitrary constant coefficients $a, b, \ldots$. The scalar potential $U\left(x_{1}, x_{3}\right)$ is a polynomial of sixth order in coordinates (see details in [69]) - [71]). Due to results of the previous Subsection, the spectral problem for matrix $2 \times 2$ Pauli Hamiltonian is reduced to a couple of spectral problems with scalar Hamiltonians $h^{(0)}, h^{(2)}$, which is much simpler. This example demonstrates the opportunity of dynamical supersymmetric diagonalization of a class of matrix problems by means of two-dimensional SUSY Quantum Mechanical approach. The identification of Pauli operator $H_{P}$ with matrix component of superHamiltonian is possible for much wider class of external fields, if some unitary rotation is preliminary done (more details in [69] - [71]).

It is instructive to notice that this method works for a wide class of nonhomogeneous magnetic fields which can be directed at any angle with respect to plane, including the field parallel to the plane. The magnetic dipole moment $\mu$ of the particle is also arbitrary. It is necessary to mention the different supersymmetric approach to solution of Pauli equation for the fermion in the plane [110], [111, [112], [113], which works only for gyromagnetic ratio equal 2 and only for (non-homogeneous) magnetic fields directed orthogonally to the plane. The latter condition in itself provides the diagonalizability of the problem, but SUSY allows to study the symmetry properties and the spectrum of Pauli operator. The method of [110], [111, [112] basically has the form of one-dimensional SUSY Quantum Mechanics but with matrix $2 \times 2$ supercharge operators $Q^{ \pm}$(see also [114]). The initially diagonalizable Pauli equation for fermions with even integer giromagnetic ratios in some specific magnetic fields orthogonal to the plane was considered also in [115] in the framework of nonlinear SUSY. 
2. The second illustrative two-dimensional model [36] corresponds to the initial potential of Coulomb form,

$$
V^{(0)}(\vec{x})=-\frac{\alpha}{r} ; \quad r^{2}=x_{1}^{2}+x_{2}^{2} ; \quad \alpha>0 .
$$

In this case, the superpotential can be taken as $W=2 \alpha r$, and therefore, the component $V^{(2)}$ has no bound states at all,

$$
V^{(2)}(\vec{x})=+\frac{\alpha}{r} .
$$

These specific circumstances lead to equivalence of the spectra of matrix Hamiltonian operator and scalar component of super-Hamiltonian $h^{(0)}(\vec{x})$. Since the discrete spectrum of the latter is well known, the spectrum of the matrix Hamiltonian with potential,

$$
V_{i k}^{(1)}(\vec{x})=-\frac{\alpha}{r}\left(2 \frac{x_{i} x_{k}}{r^{2}}-\delta_{i k}\right) .
$$

is known as well. Its wave functions can be obtained from the wave functions of scalar Coulomb problem $V^{(0)}$ by means of intertwining relations (18) with operators $q_{l}^{-}$. The potential (31) can be physically interpreted in curvilinear (polar) coordinates [202]. It corresponds to attractive Coulomb potential for $1 / 2$-spin particle with positive spin projection $s_{z}=1 / 2$ and to repulsive Coulomb - for particle with negative $s_{z}=-1 / 2$.

\subsection{SUSY QM in arbitrary dimensionality of space as $(0+1)$-dimensional Quantum} Field Theory

Both one-dimensional Factorization Method and its two-dimensional generalization were shown above to realize the simplest superalgebra (12) of Supersymmetric Quantum Mechanics. This observation gives the idea how we could generalize this approach further for arbitrary dimensionality $d$ of space: it is necessary to build new realizations of the same SUSY algebra (12). For this purpose it is helpful 68 to exploit some methods and experience of well-developed Supersymmetric Quantum Field Theory [116]. Indeed, new such realizations of the SUSY algebra was obtained [68] by study of some simple models of SUSY Quantum Field Theory. The simplest QFT, which can be interpreted as nonrelativistic Quantum Mechanics, is the theory in $(0+1)$ - dimensional space, i.e. fields depend on one variable - the time $t$. Keeping in mind, that supersymmetry must be a property of the model, we consider the superfield, which depends both on one bosonic coordinate (time $t$ ) and a pair of mutually conjugate Grassman variables $\theta, \bar{\theta}$. The dimensionality $d$ of SUSY Quantum Mechanics on target is provided by choosing the fields real, but with $d$-components.

Thus, the main ingredient of the model is [68] the $d$-dimensional real superfield,

$$
\begin{aligned}
& \varphi_{l}(t ; \theta, \bar{\theta}) ; \quad l=1,2, \ldots, d ; \quad \varphi_{l}=\bar{\varphi}_{l} ; \\
& \{\theta, \theta\}=\{\bar{\theta}, \bar{\theta}\}=\{\theta, \bar{\theta}\}=[t, \theta]=[t, \bar{\theta}]=0 .
\end{aligned}
$$

As it is typical for the genuine superfield theory, the infinitesimal super-transformation in the space $(t ; \theta, \bar{\theta})$ is defined as,

$$
t \rightarrow t^{\prime}=t-i(\bar{\theta} \epsilon-\bar{\epsilon} \theta) ; \quad \theta \rightarrow \theta^{\prime}=\theta+\epsilon ; \quad \bar{\theta} \rightarrow \bar{\theta}^{\prime}=\bar{\theta}+\bar{\epsilon},
$$


with a pair of Grassman mutually conjugate parameters of transformation $\epsilon, \bar{\epsilon}$. The "infinitesimal" (linear in $\epsilon, \bar{\epsilon}$ ) transformations (34) can be generated by differential operators (super-generators) in the superspace $(t ; \theta, \bar{\theta})$,

$$
Q=i \frac{\partial}{\partial \theta}-\bar{\theta} \frac{\partial}{\partial t} ; \quad \bar{Q}=-i \frac{\partial}{\partial \bar{\theta}}+\theta \frac{\partial}{\partial t}
$$

which produce the finite transformations by,

$$
U=\exp [i(\bar{\epsilon} \bar{Q}+Q \epsilon)] .
$$

Operators $Q, \bar{Q}$ satisfy the anticommutation relations,

$$
\{Q, Q\}=\{\bar{Q}, \bar{Q}\}=0 ; \quad\{Q, \bar{Q}\}=i \frac{\partial}{\partial t} .
$$

Due to nilpotency of $\theta, \bar{\theta}$, the bosonic superfield can be expanded as,

$$
\varphi_{l}(t ; \theta, \bar{\theta})=x_{l}(t)+i \theta \psi_{l}(t)-i \bar{\psi}_{l}(t) \bar{\theta}+\bar{\theta} \theta D_{l}(t),
$$

where $x_{l}(t), D_{l}(t)$ are bosonic real $d$-component fields, and $\psi_{l}(t), \bar{\psi}_{l}(t)$ - mutually conjugate $d$-component fermionic (anticommuting) fields. Restricting ourselves to superscalar fields,

$$
\varphi_{l}^{\prime}\left(t^{\prime} ; \theta^{\prime}, \bar{\theta}^{\prime}\right)=\varphi_{l}(t ; \theta, \bar{\theta}),
$$

one obtains the following rules of transformation,

$$
\begin{array}{ll}
\delta x_{l}(t)=i \epsilon \psi_{l}(t)-i \bar{\psi}_{l}(t) \bar{\epsilon} ; & \delta D_{l}(t)=\bar{\epsilon} \dot{\bar{\psi}}_{l}(t)+\dot{\psi}_{l}(t) \epsilon \\
\delta \psi_{l}(t)=i \bar{\epsilon} D_{l}(t)-\bar{\epsilon} \dot{x}_{l}(t) ; & \delta \bar{\psi}_{l}(t)=-i \epsilon D_{l}(t)-\epsilon \dot{x}_{l}(t),
\end{array}
$$

where dot means derivative over $t$.

As usual, for construction of superinvariant action functional we need the operators of supercovariant derivatives,

$$
D=\frac{\partial}{\partial \theta}-i \bar{\theta} \frac{\partial}{\partial t} ; \quad \bar{D}=\frac{\partial}{\partial \bar{\theta}}-i \theta \frac{\partial}{\partial t}
$$

which allow to build the superinvariant action,

$$
S=\int d t L(\vec{\varphi})=\int d t \int d \theta d \bar{\theta}(-\bar{D} \vec{\varphi} D \vec{\varphi}+W(\vec{\varphi}))
$$

with arbitrary functional $W(\vec{\varphi})$ - superpotential of the model. Expanding it around the point $x_{l}(t)$, integrating the action (42) over both Grassmann variables and substituting nondynamical variables $D_{l}, \bar{D}_{l}$ from the corresponding equations of motion, one obtains the action as functional of component fields and superpotential,

$S=\int d t\left(\dot{x}_{l} \dot{x}_{l}+i \bar{\psi}_{l} \dot{\psi}_{l}-i \dot{\bar{\psi}}_{l} \psi_{l}-\left(\partial_{l} W\right)(\vec{x})\left(\partial_{l} W\right)(\vec{x})-\left(\partial_{l} \partial_{m} W\right)(\vec{x})\left[\bar{\psi}_{l}(t), \psi_{m}(t)\right]\right)$.

The corresponding classical Hamiltonian is,

$$
H_{c l}=p_{l} p_{l}+\left(\partial_{l} W\right)\left(\partial_{l} W\right)+\left(\partial_{l} \partial_{m} W\right)\left[\bar{\psi}_{l}, \psi_{m}\right]
$$

where $p_{l}$ is the momentum $p_{l}=\delta L / \delta \dot{x}_{l}=\dot{x}_{l}$. According to Noether theorem (in the supersymmetric context), supercharges in the present field theory are [117],

$Q_{c l}^{+}=\frac{\delta L}{\delta \dot{\epsilon}}=\left(i p_{l}+\left(\partial_{l} W\right)\right) \psi_{l} ; \quad Q_{c l}^{-}=\frac{\delta L}{\delta \dot{\bar{\epsilon}}}=\left(-i p_{l}+\left(\partial_{l} W\right)\right) \bar{\psi}_{l}=\left(Q^{+}\right)^{\dagger}$. 
To present point, the model was formulated on the classical level, but now everything is prepared for its quantization (the prescriptions for canonical quantization of mixed systems both with bosonic and fermionic degrees of freedom see in [118]). After quantization, coordinates $x_{l}$, momenta $p_{l}$ and fermionic variables $\psi_{l}, \bar{\psi}_{l}$ become the operators $\hat{x}_{l}, \hat{p}_{l}, b_{l}^{+}, b_{l}^{-}$, correspondingly,

$$
\left[\hat{p}_{l}, \hat{x}_{m}\right]=-i \delta_{l m} ; \quad\left\{b_{l}^{+}, b_{m}^{-}\right\}=\delta_{l m} ; \quad\left\{b_{l}^{+}, b_{m}^{+}\right\}=\left\{b_{l}^{-}, b_{m}^{-}\right\}=0,
$$

and anticommuting operators $b_{l}^{ \pm}$can be interpreted as creation and annihilation operators for the system of $d$ localized scalar particles, but with Fermi statistics. The quantized super-Hamiltonian has the form,

$$
H=\hat{p}_{l} \hat{p}_{l}+\left(\partial_{l} W\right)\left(\partial_{l} W\right)+\left(\partial_{l} \partial_{m} W\right) b_{l}^{+} b_{m}^{-},
$$

and quantum supercharges are,

$$
Q^{ \pm}=\left( \pm i \hat{p}_{l}+\left(\partial_{l} W\right)(\vec{x})\right) b_{l}^{ \pm}
$$

These quantum operators satisfy all relations of superalgebra (12), and they provide the required generalization of one- and two-dimensional supersymmetric models of Introduction and Subsection 2.1 to the case of arbitrary dimensionality $d$.

\subsection{The structure of super-Hamiltonian in SUSY QM for arbitrary dimensionality of space}

In order to understand the structure of $d$-dimensional quantum super-Hamitlonian (47) and supercharges (48), it is useful [68] to use the fermion number operator,

$$
N_{F}=b_{l}^{+} b_{l}^{-} \text {, }
$$

which commutes with $H, Q^{ \pm}$as follows,

$$
\left[H, N_{F}\right]=0 ; \quad\left[N_{F}, Q^{ \pm}\right]= \pm Q^{ \pm} .
$$

If the basis in the Hilbert space $\mathcal{H}$ of states is chosen so that the space is a direct sum of subspaces $\mathcal{H}_{n}$ with fixed number $n$ of fermions $(n=0,1,2, \ldots, d)$, the conservation law (50) means that super-Hamiltonian has the block-diagonal form in the fermionic Fock space,

$$
H=\left(\begin{array}{cccc}
h^{(0)} & 0 & 0 & 0 \\
0 & h^{(1)} & 0 & 0 \\
0 & 0 & \cdots & 0 \\
0 & 0 & 0 & h^{(d)}
\end{array}\right)
$$

with components $h^{(n)}$ acting in the subspace $\mathcal{H}_{n}$. The convenient basis in $\mathcal{H}_{n}$ is the basis of occupation numbers - $C_{d}^{n}$ vectors $\mid n_{1}, n_{2}, \ldots, n_{d}>$ with $n_{l}=0,1$, and $\sum_{l=1}^{l=d} n_{l}=n$. In turn, the supercharge $Q^{+}$has the block structure with nonvanishing matrix $C_{d}^{n+1} \times C_{d}^{n}$ 
blocks $q_{n, n+1}^{+}$below the main diagonal, and $Q^{-}$- matrix $C_{d}^{n} \times C_{d}^{n+1}$ blocks $q_{n+1, n}^{-}$above the main diagonal,

$$
Q^{+}=\left(\begin{array}{ccccc}
0 & 0 & 0 & 0 & 0 \\
q_{0,1}^{+} & 0 & 0 & 0 & 0 \\
0 & q_{1,2}^{+} & 0 & 0 & 0 \\
0 & 0 & \ldots & 0 & 0 \\
0 & 0 & 0 & q_{d-1, d}^{+} & 0
\end{array}\right) ; \quad Q^{-}=\left(\begin{array}{ccccc}
0 & q_{1,0}^{-} & 0 & 0 & 0 \\
0 & 0 & q_{2,1}^{-} & 0 & 0 \\
0 & 0 & 0 & \ldots & 0 \\
0 & 0 & 0 & 0 & q_{d, d-1}^{-} \\
0 & 0 & 0 & 0 & 0
\end{array}\right) \text {. }
$$

The algebra (12) and matrix representations (51), (52) provide the following relations,

$$
h^{(n)}=q_{n-1, n}^{+} q_{n, n-1}^{-}+q_{n+1, n}^{-} q_{n, n+1}^{+} \equiv \underline{h}^{(n)}+\underline{\underline{h}}^{(n)}
$$

(quasifactorization - compare with (15)),

$$
q_{n+1, n+2}^{+} q_{n, n+1}^{+}=q_{n-1, n-2}^{-} q_{n, n-1}^{-}=0 ; \quad \underline{h}^{(n)} \underline{\underline{h}}^{(n)}=\underline{\underline{h}}^{(n)} \underline{h}^{(n)}=0,
$$

(orthogonality - compare with the orthogonality $p_{l}^{ \pm} q_{l}^{\mp}=q_{l}^{ \pm} p_{l}^{\mp}=0$ in Subsection 2.1),

$$
\begin{aligned}
& \underline{\underline{h}}^{(n+1)} q_{n, n+1}^{+}=q_{n, n+1}^{+} \underline{\underline{h}}^{(n)}=\underline{h}^{(n)} q_{n+1, n}^{-}=q_{n+1, n}^{-} \underline{\underline{h}}^{(n+1)}=0 ; \\
& \underline{\underline{h}}^{(n+1)} q_{n, n+1}^{+}=q_{n, n+1}^{+} \underline{\underline{h}}^{(n)} ; \quad q_{n+1, n}^{-} \underline{\underline{h}}^{(n+1)}=\underline{\underline{h}}^{(n)} q_{n+1, n}^{-},
\end{aligned}
$$

(intertwining relations analogous to (4)).

Thus, each matrix component $h^{(n)},(n=1, \ldots, d-1)$ of the super-Hamiltonian $H$ contains two mutually orthogonal terms $\underline{\underline{h}}^{(n)}, \underline{h}^{(n)}$. These terms are intertwined with analogous terms of neighbor matrix components, providing their spectral equivalence (again, up to zero modes of supercharges). Due to orthogonality (54), each matrix component $h^{(n)}$, as a whole, is also intertwined with neighbor $h^{(n-1)}$ and $h^{(n+1)}$. As for connection of the wave functions, those of $h^{(n)}$ are connected, analogously to (5) and (20) with wave functions of neighbor Hamiltonians $h^{(n+1)}, h^{(n-1)}$. This scheme can be illustrated by the following diagram,

$$
\left[h^{(0)}\right] \leftrightarrows\left[\underline{h}^{(1)}+\underline{\underline{h}}^{(1)}\right] \leftrightarrows\left[\underline{h}^{(2)}+\underline{\underline{h}}^{(2)}\right] \leftrightarrows \ldots\left[\underline{h}^{(d-1)}+\underline{\underline{h}}^{(d-1)}\right] \leftrightarrows h^{(d)}
$$

Thereby, the structure of elements of SUSY QM for arbitrary dimensionality of space is known. In order to obtain the explicit expressions for potentials, one has to choose any matrix representation for the fermionic creation and annihilation operators $b^{ \pm}$. In particular, choosing

$$
b^{+}=\left(\begin{array}{cc}
0 & 0 \\
1 & 0
\end{array}\right) ; \quad b^{-}=\left(\begin{array}{cc}
0 & 1 \\
0 & 0
\end{array}\right),
$$

the relations of the Introduction for $d=1$ are obtained. For the space dimensionality $d=2$, the suitable representation for fermionic creation and annihilation operators $b_{l}^{ \pm}, l=1,2$ is $4 \times 4$ matrix,

$$
b_{1}^{+}=\left(\begin{array}{cccc}
0 & 0 & 0 & 0 \\
1 & 0 & 0 & 0 \\
0 & 0 & 0 & 0 \\
0 & 0 & -1 & 0
\end{array}\right)=\left(b_{1}^{-}\right)^{\dagger} ; \quad b_{2}^{-}=\left(\begin{array}{cccc}
0 & 0 & 0 & 0 \\
0 & 0 & 0 & 0 \\
1 & 0 & 0 & 0 \\
0 & 1 & 0 & 0
\end{array}\right)=\left(b_{2}^{-}\right)^{\dagger},
$$

leading [68] to the same $4 \times 4$ matrix dimensionality of the supercharges $Q^{ \pm}$and the super-Hamiltonian $H$ given by (21). 


\subsection{The particular case of $d=3:$ nucleon-nucleon interaction}

For physically interesting case of $d=3$, three creation and annihilation operators $b_{l}^{ \pm}, l=1,2,3$, and the supercharges $Q^{ \pm}$are of matrix dimensionality $8 \times 8$. The superHamiltonian $H$ includes 68 four components: two scalars $h^{(0)}, h^{(3)}$ and two $3 \times 3$ matrices $h_{i k}^{(1)}, h_{i k}^{(2)}$. According to the general scheme of previous Subsection, there are intertwining relations in the pairs $h^{(0)} \rightleftharpoons h_{i k}^{(1)} ; h_{i k}^{(1)} \rightleftharpoons h_{i k}^{(2)} ; h_{i k}^{(2)} \rightleftharpoons h^{(3)}$, providing the relations between corresponding scalar and vector (three-component) wave functions. In this case, the same commutation and anticommutation relations of the superalgebra (12) are fulfilled.

The possibility of using of this $8 \times 8$ realization of (12) can be illustrated by derivation [72] of simple connections between different sectors of nucleon-nucleon and nucleon-antinucleon interactions in the OPEP (one-pion-exchange-potential) approximation. The general form of the Hamiltonian of interaction of two nonrelativistic particles of spin $1 / 2$ with scalar, spin-spin and tensor forces is:

$H(1 / 2 \otimes 1 / 2)=-\frac{1}{2} \Delta^{(3)}+V_{S}(r)+\frac{1}{2}\left(1+\vec{\sigma}_{1} \vec{\sigma}_{2}\right) V_{\sigma}(r)+\left(\left[3\left(\vec{\sigma}_{1} \vec{\partial}\right) 3\left(\vec{\sigma}_{2} \vec{\partial}\right)-\left(\vec{\sigma}_{1} \vec{\sigma}_{2}\right) \Delta^{(3)}\right] V_{T}\right)(r)$,

where $\vec{\sigma}_{1}, \vec{\sigma}_{2}$ are Pauli matrices describing spin operators of two nucleons. Separating (59) onto singlet spin $(S=0)$ and triplet $\operatorname{spin}(S=1)$ parts,

$V^{S=0}=V_{S}(r)-V_{\sigma}(r) ; \quad V_{l k}^{S=1}=\left(V_{S}+V_{\sigma}+2 \Delta^{(3)} V_{T}\right) \delta_{l k}-6\left(\partial_{l} \partial_{k} V_{T}\right) ; l, k=1,2,3,(60)$

and choosing suitably the spherically symmetric superpotential $W(r)$, one may identify the first part $V^{S=0}$ with the scalar potential of the component $h^{(0)}$ of the superHamiltonian:

$$
V^{S=0}=\frac{1}{2}\left[(\vec{\partial} W)^{2}(r)-\left(\Delta^{(3)} W\right)(r)\right]=\frac{1}{2}\left[\left(W^{\prime}\right)^{2}-W^{\prime \prime}+\frac{2}{r} W^{\prime}\right],
$$

where prime means derivative over $r$. Simultaneously, it is possible [72 to identify the triplet spin part $V_{l k}^{S=1}$ with the $3 \times 3$ component $h_{l k}^{(2)}$ of the super-Hamiltonian, i.e. to put:

$V_{T}(r)=\frac{1}{6} W(r) ; \quad V_{\sigma}(r)=\frac{1}{3}\left(\Delta^{(3)} W\right)(r) ; \quad V_{S}(r)=\frac{1}{2}(\vec{\partial} W)^{2}(r)-\frac{1}{6}\left(\Delta^{(3)} W\right)(r)$.

In the OPEP-approximation, the explicit form of $N N$ interaction potential is:

$$
V^{N N}=f^{2}\left(\vec{\tau}_{1} \vec{\tau}_{2}\right)\left(\vec{\sigma}_{1} \vec{\partial}\right)\left(\vec{\sigma}_{2} \vec{\partial}\right) \frac{e^{-\mu r}}{r},
$$

where $\vec{\tau}_{1}, \vec{\tau}_{2}$ are isospin operators of nucleons, $f^{2}$ is a coupling constant, and $\mu$ - pion mass. The expression (63) corresponds to the specific forms of tensor, spin-spin and scalar terms:

$$
V_{T}^{N N}=\frac{1}{3} f^{2}\left(\vec{\tau}_{1} \vec{\tau}_{2}\right) \frac{e^{\mu r}}{r} ; \quad V_{\sigma}^{N N}=2 \Delta^{(3)} V_{T}^{N N} ; \quad V_{S}^{N N}=-\Delta^{(3)} V_{T}^{N N} .
$$

Choosing the superpotential $W$ in the super-Hamiltonian $H$ as

$$
W(r)=2\left(\vec{\tau}_{1} \vec{\tau}_{2}\right) f^{2} \frac{e^{\mu r}}{r},
$$


one can identify [72] the Hamiltonian $H^{N N}$ with $h^{(0)} \bigoplus h_{l k}^{(2)}$ with exponential accuracy $\left(W^{\prime}\right)^{2} / W^{\prime \prime}$ (see [119]).

It is important that in the OPEP-approximation, two other components $h_{l k}^{(1)}$ and $h^{(3)}$ of the super-Hamiltonian $H$ have also simple physical sense (see [72]): they describe the triplet spin $S=1$ and singlet spin $S=0$, correspondingly, of nucleon-antinucleon interaction $H^{N \bar{N}}=h_{l k}^{(1)} \bigoplus h^{(3)}$. Thus, just the parts of $H^{N N}$ and $H^{N \bar{N}}$ with definite total spin being the components of the same super-Hamiltonian satisfy the supersymmetric intertwining relations of the form (55). In case, if there were bound $N N$ states, their spectra would (almost) coincide, and we could write the relations between the wave functions $\Psi_{n}^{N N}$ and $\Psi_{n}^{N \bar{N}}$, similar to (20). In the present case, supersymmetrical intertwining relations allow to connect asymptotic behaviour of the wave functions of continuous spectra. The non-stationary and stationary non-relativistic scattering theory for a supersymmetrical Hamiltonian was developed in [72], where the simple connections between scattering data - partial amplitudes, phase shifts and mixing parameters - of the components of super-Hamiltonian were derived.

\section{Polynomial SUSY in $d=1$}

\subsection{Supercharges of higher order in derivatives from ladder construction}

The supersymmetric methods of the previous section was based on the superalgebra (12) which includes: supersymmetry, i.e. conservation of the supercharges $Q^{ \pm}$, (quasi)factorization of the super-Hamiltonian components, and the nilpotency of supercharges which is provided by their matrix form. Just the supersymmetry (i.e. the intertwining relations) guarantees the (almost exact) isospectrality between the components of super-Hamiltonian with non-singular potentials, providing the most interesting practical results of the method. This is a reason to consider generalized SUSY with supercharges of higher order in derivatives, and therefore, without quasifactorization of Hamiltonians in terms of components of supercharges. In the context of Darboux transformation [19], such a generalization of the intertwining relations (4) corresponds to the so called Crum transformation [22]:

$$
h^{(0)} q_{N}^{+}=q_{N}^{+} h^{(1)}, \quad q_{N}^{-} h^{(0)}=h^{(1)} q_{N}^{-},
$$

where $N$-th order differential operators are:

$$
q_{N}^{ \pm}=\sum_{k=0}^{N} w_{k}^{ \pm}(x) \partial^{k}, \quad w_{N}^{ \pm} \equiv(\mp 1)^{N}
$$

with differentiable coefficient functions. Herein let us take them real and the pairs of intertwining operators to be mutually Hermitian conjugated $q_{N}^{-}=\left(q_{N}^{+}\right)^{\dagger}$.

In the literature there are several synonyms for the higher-order SUSY algebra: originally it was named as a Polynomial (or Higher-derivative) one [46, 47], later a more general term of Nonlinear SUSY has been used [106, 120] in a certain relationship to a 
nonlinear SUSY algebra arising in the conformal QM [121]. The title of $N$-fold SUSY has been also suggested in [65].

One way to achieve the non-linear SUSY is in combining of several linear $\mathcal{N}=1$ SUSY QM systems in the fermion number representation which are implemented by nilpotent supercharges of first order in derivatives (2) built from a real super-potentials $W(x)$,

$q_{1}^{ \pm} \equiv r^{ \pm}=\mp \partial+\chi(x) ; \quad \chi(x) \equiv(\partial W)(x)$

In the latter case the SUSY algebra is completed (12) by the appropriate factorization (1), (3) of the super-Hamiltonian [34, 35].

Let us proceed by recursion: from a simple Darboux transformation to a ladder [37] or a dressing chain [122] made of several simple Darboux steps. To produce the required transformation operators the two different linear SUSY systems may be overlapped. Namely, consider two super-Hamiltonians $H_{i}, i=1,2$, Eq. (11), respectively two sets of supercharges $Q_{i}^{ \pm}$with coefficient functions $\chi_{i}=\left(\partial W_{i}\right)$ and supercharge components $r_{i}^{ \pm}=\mp \partial+\chi_{i}$. Let us match two elements of super-Hamiltonians with a constant shift,

$$
h_{1}^{(1)}=h_{2}^{(0)}+\lambda ; \quad \chi_{1}^{2}+\chi_{1}^{\prime}=\chi_{2}^{2}-\chi_{2}^{\prime}+\lambda .
$$

Evidently, the ground state energies for the above Hamiltonians $\bar{E}_{1}, \bar{E}_{2}$ satisfy the inequalities $\bar{E}_{1} \geq \lambda \geq-\bar{E}_{2}$. The constant shift of the super-Hamiltonian $H_{2} \rightarrow H_{2}+\lambda$ does not break or change its supersymmetry but modify the superalgebra.

After matching the chain of intertwining relations (4) can be assembled to produce the final Hamiltonian $h_{2}^{(1)}$ only from the initial one, $h_{1}^{(0)}$ by means of a second-order Darboux transformation,

$h_{1}^{(0)} r_{1}^{+} r_{2}^{+}=r_{1}^{+} h_{1}^{(1)} r_{2}^{+}=r_{1}^{+}\left(h_{2}^{(0)}+\lambda\right) r_{2}^{+}=r_{1}^{+} r_{2}^{+}\left(h_{2}^{(1)}+\lambda\right) ; \quad r_{2}^{-} r_{1}^{-} h_{1}^{(0)}=\left(h_{2}^{(1)}+\lambda\right) r_{2}^{-} r_{1}^{-}$.

Following the spectral design one concludes that the two-step ladder SUSY dynamics contains the redundant information, namely, about the intermediate Hamiltonians $h_{1}^{(1)}=h_{2}^{(0)}+\lambda$. Let us remove it and define the two (almost) isospectral components,

$$
\begin{aligned}
& h^{(0)} \equiv h_{1}^{(0)}+\lambda_{1}=r_{1}^{+} r_{1}^{-}+\lambda_{1} ; \quad h^{(1)} \equiv h_{2}^{(1)}+\lambda_{2}=r_{2}^{-} r_{2}^{+}+\lambda_{2} ; \\
& r_{1}^{-} r_{1}^{+}+\lambda_{1}=r_{2}^{+} r_{2}^{-}+\lambda_{2} ;
\end{aligned}
$$

for the super-Hamiltonian (11) for which we have employed an energy reference shifted by arbitrary $\lambda_{1,2}, \lambda=\lambda_{2}-\lambda_{1}$. Then the intertwining relations (66) are identical to Eq. (170) with $q_{2}^{+}=r_{1}^{+} r_{2}^{+}=\left(q_{2}^{-}\right)^{\dagger}$ and the supersymmetry $\left[H, Q_{2}^{+}\right]=\left[H, Q_{2}^{-}\right]=0$ is generated by the conserved nilpotent supercharges,

$$
Q_{2}^{+}=\left(\begin{array}{cc}
0 & 0 \\
q_{2}^{-} & 0
\end{array}\right)=\left(Q_{2}^{-}\right)^{\dagger}, \quad\left(Q_{2}^{+}\right)^{2}=\left(Q_{2}^{-}\right)^{2}=0
$$

Now in virtue of (171) the algebraic closure of the SUSY algebra is given by,

$$
\left\{Q_{2}^{+}, Q_{2}^{-}\right\}=\left(\begin{array}{cc}
r_{1}^{+} r_{2}^{+} r_{2}^{-} r_{1}^{-} & 0 \\
0 & r_{2}^{-} r_{1}^{-} r_{1}^{+} r_{2}^{+}
\end{array}\right)=\left(H-\lambda_{1}\right)\left(H-\lambda_{2}\right),
$$


generalizing Eq.(12). Thus we have obtained the second-order Polynomial SUSY algebra [46]-[51] as a concise form of isospectral deformation of a potential system realized by a ladder [33]-43] or a dressing chain [122]-125] of a couple of one-step Darboux transformations or, equivalently, by a second-order Crum-Darboux intertwining [22] or by a blocking of two linear SUSY with partial overlapping of super-Hamiltonians [46] ("weak SUSY" [126]), or by a tower of para-SUSY transformations [127]-[131] (see how to proceed in [14]) or by a reduction of spin-j parasupersymmetry [132].

The formal zero-modes $₫$ of intertwining operators $q_{2}^{ \pm}$form the basis of a twodimensional representation of the super-Hamiltonian,

$$
\begin{aligned}
& h^{(0)} q_{2}^{+} \phi_{i}^{+}(x)=0=q_{2}^{+} h^{(1)} \phi_{i}^{+}(x) ; \quad i=1,2 ; \\
& h^{(1)} \phi_{i}^{+}(x)=\sum_{j=1}^{2} S_{i j}^{-} \phi_{j}^{+}(x) ; \quad h^{(0)} \phi_{i}^{-}(x)=\sum_{j=1}^{2} S_{i j}^{+} \phi_{j}^{-}(x),
\end{aligned}
$$

due to intertwining relations (66), (70). In terms of these Hamiltonian projections constant matrices $S^{ \pm}$, the SUSY algebra closure takes the polynomial form [73] (see also [65,

$$
\left\{Q_{2}^{+}, Q_{2}^{-}\right\}=\operatorname{det}\left[E \mathbf{I}-S^{+}\right]_{E=H}=\operatorname{det}\left[E \mathbf{I}-S^{-}\right]_{E=H} \equiv \mathcal{P}_{2}(H) .
$$

Thus both matrices have the same set of eigenvalues which for the ladder construction (74) consists of $\lambda_{1}, \lambda_{2}$. As the formal zero-mode set is not uniquely derived from (75) the matrices $S^{ \pm}$are not necessarily diagonal. For instance, the equation $r_{1}^{+} r_{2}^{+} \phi^{+}(x)=$ 0 has one formal zero-mode $\phi_{2}^{+}$obeying $r_{2}^{+} \phi_{2}^{+}(x)=0$ and another one obeying $r_{1}^{+} \tilde{\phi}_{1}^{+}=0 ; \tilde{\phi}_{1}^{+}=r_{2}^{+} \phi_{1}^{+}(x) \neq 0$. Evidently the zero-mode solution $\phi_{1}^{+}(x)$ is determined up to an addition of $\phi_{2}^{+}$. When multiplying these linear equations by $r_{2}^{-}$one proves with the help of Eqs. (71), (72) that

$$
\left(h^{(1)}-\lambda_{2}\right) \phi_{2}^{+}(x)=0 ; \quad\left(h^{(1)}-\lambda_{1}\right) \phi_{1}^{+}(x)=C \phi_{2}^{+}(x) ; \quad S^{-}=\left(\begin{array}{cc}
\lambda_{1} & C \\
0 & \lambda_{2}
\end{array}\right),
$$

where $C$ is an arbitrary real constant. If $\lambda_{1} \neq \lambda_{2}$ then by the redefinition $\left(\lambda_{1}-\lambda_{2}\right) \bar{\phi}_{1}^{+} \equiv\left(\lambda_{1}-\lambda_{2}\right) \phi_{1}^{+}+C \phi_{2}^{+}(x)$ one arrives to the diagonal matrix $S^{-}$. However in the confluent case, $\lambda_{1}=\lambda_{2} \equiv \lambda, C \neq 0$ it is impossible to diagonalize and by a proper normalization of the zero-mode $\phi_{1}^{+}$one gets the elementary Jordan cell,

$$
\left(h^{(1)}-\lambda\right) \phi_{2}^{+}(x)=0 ; \quad\left(h^{(1)}-\lambda\right) \phi_{1}^{+}(x)=\phi_{2}^{+}(x) ; \quad S^{-}=\left(\begin{array}{cc}
\lambda & 1 \\
0 & \lambda
\end{array}\right) .
$$

Therefore in the confluent case the zero-mode $\phi_{1}^{+}$is not anymore a solution of the Schrödinger equation but it is an adjoint solution which can be derived by differentiation of the first relation in (78),$\phi_{1}^{+}=d \phi_{2}^{+} / d \lambda+c_{1} \phi_{2}^{+}$where $c_{1}$ is an arbitrary constant. Still the intermediate Hamiltonian $\tilde{h}=r_{1}^{-} r_{1}^{+}+\lambda=r_{2}^{+} r_{2}^{-}+\lambda$ is well defined and therefore the intermediate isospectral partner $\tilde{\phi}_{1}^{+}(x)$ of the zero-mode $\phi_{1}^{+}(x)$ is a solution of

$\ddagger$ We mark them as "formal" because they are not supposed to be normalizable, just being solutions of the Schrödinger equation but not necessarily belonging to the set of spectrum eigenfunctions. 
Schrödinger equation with the above Hamiltonian. The analysis of the matrix $S^{+}$is similar. Thus we convince ourselves that in general the Hamiltonian projection onto the subspace of supercharge zero-modes is not diagonalizable but can be always transformed into a canonical Jordan form.

To complete the description of Polynomial SUSY algebras generated by a secondorder ladder one should take into consideration also the degenerate case when $\lambda_{1}=\lambda_{2} \equiv \lambda, C=0$. For this choice the matrix $S^{-}$is automatically diagonal and both zero modes $\phi_{1,2}^{+}(x)$ are (independent) solutions of the Schrödinger equation with the Hamiltonian $h^{-}$. Then it can be proved [73] that the intertwining operator $q_{2}^{+}$is just a linear function of this Hamiltonian, $q_{2}^{+}=\lambda-h^{(1)}$. Hence the intertwining is trivial $h^{(1)}=h^{(0)}$ and such supercharges must be ruled out due to their triviality. However for SUSY of higher order $N \geq 3$ the removal of such blocks from supercharges may lead to less-dimensional, ladder-irreducible SUSY algebras (see Subsection 3.3).

\subsection{Polynomial SUSY QM from a ladder of linear SUSY systems}

Let us give the general description of the $N$-step ladder of linear SUSY algebras which entails the Polynomial Superalgebra of $N$ th-order [46], [47]. We introduce a set of first-order differential operators for intermediate intertwinings ,

$$
r_{l}^{ \pm}=\mp \partial+\chi_{l}(x), \quad l=1, \ldots, N,
$$

and the relevant number of intermediate coefficient functions $\chi_{l}(x)=\left(\partial W_{l}\right)$ related to intermediate superpotentials $W_{l}(x)$. The set of the initial, $h^{(0)} \equiv h_{0}$, the final, $h^{(1)} \equiv h_{N}$ and intermediate Hamiltonians, $h_{l}=-\partial^{2}+v_{l}(x)$ consists of Schrödinger operators, nonsingular and real ones. They obey the matching (ladder) relations,

$$
\begin{aligned}
& h_{l} \equiv r_{l}^{-} \cdot r_{l}^{+}+\lambda_{l}=r_{l+1}^{+} \cdot r_{l+1}^{-}+\lambda_{l+1}, \quad l=1, \ldots, N-1, \\
& h_{0} \equiv h^{(0)}=r_{1}^{+} \cdot r_{1}^{-}+\lambda_{1} ; \quad h_{N} \equiv h^{(1)}=r_{N}^{-} \cdot r_{N}^{+}+\lambda_{N} .
\end{aligned}
$$

These ladder relations correspond to the (dressing) chain equations on super-potentials, $W_{l}(x), \chi_{l}(x)=\left(\partial W_{l}\right)(x)$

$$
v_{l}(x)=\left(\chi_{l}(x)\right)^{2}+\left(\chi_{l}(x)\right)^{\prime}+\lambda_{l}=\left(\chi_{l+1}(x)\right)^{2}-\left(\chi_{l+1}(x)\right)^{\prime}+\lambda_{l+1}
$$

The corresponding intertwining (Darboux) transformations hold in each adjacent pair of Hamiltonians,

$$
h_{l-1} \cdot r_{l}^{+}=r_{l}^{+} \cdot h_{l}, \quad r_{l}^{-} \cdot h_{l-1}=h_{l} \cdot r_{l}^{-},
$$

and therefore the chain of $N$ overlapping SUSY systems is properly built,

$$
\begin{aligned}
& H_{l}=\left(\begin{array}{cc}
h_{l-1} & 0 \\
0 & h_{l}
\end{array}\right), \quad R_{l}^{-}=\left(\begin{array}{cc}
0 & r_{l}^{+} \\
0 & 0
\end{array}\right)=\left(R_{l}^{+}\right)^{\dagger} ; \\
& {\left[H_{l}, R_{l}^{+}\right]=\left[H_{l}, R_{l}^{-}\right]=0, \quad H_{l}-\lambda_{l}=\left\{R_{l}^{+}, R_{l}^{-}\right\},}
\end{aligned}
$$

Now let us omit a chain of intermediate Hamiltonians between $h^{(0)}$ and $h^{(1)}$ and produce the Higher-derivative $\simeq$ Polynomial $\simeq$ Nonlinear SUSY algebra for the super- 
Hamiltonian $H$ given in Eq.(11). The intertwining between $h^{(0)}$ and $h^{(1)}$ is performed by the Crum-Darboux operators,

$$
q_{N}^{+}=r_{1}^{+} \ldots r_{N}^{+}, \quad q_{N}^{-}=r_{N}^{-} \ldots r_{1}^{-} .
$$

The SUSY symmetry $\left[H, Q_{N}^{+}\right]=\left[H, Q_{N}^{-}\right]=0$, is accomplished by the supercharges of the same matrix structure (73) and the super-Hamiltonian is represented by finitedimensional matrices on the subspaces of supercharge zero-modes,

$$
\begin{aligned}
& q_{N}^{ \pm} \phi_{i}^{ \pm}(x)=0 ; \quad i=1,2, \ldots, N ; \\
& h^{(0)} \phi_{i}^{-}(x)=\sum_{j=1}^{N} S_{i j}^{+} \phi_{j}^{-}(x) ; \quad h^{(1)} \phi_{i}^{+}(x)=\sum_{j=1}^{N} S_{i j}^{-} \phi_{j}^{+}(x)
\end{aligned}
$$

due to intertwining relations (66). In terms of the constant matrices $S^{ \pm}$, the algebraic closure is given by a non-linear SUSY relation [73, 65],

$$
\left\{Q_{N}^{+}, Q_{N}^{-}\right\}=\operatorname{det}\left[E \mathbf{I}-S^{+}\right]_{E=H}=\operatorname{det}\left[E \mathbf{I}-S^{-}\right]_{E=H} \equiv \mathcal{P}_{N}(H)=\prod_{l=1}^{N}\left(H-\lambda_{l}\right) .
$$

Again both matrices have the same set of eigenvalues which for the ladder construction (80) consists of $\lambda_{1}, \ldots \lambda_{N}$. If the degenerate roots appear the normal (canonical) form of matrices $S^{ \pm}$may consist of nontrivial Jordan cells. If all intermediate $h_{l}$ are hermitian, nonsingular, then $\lambda_{l}$ are real and each ladder step is well defined.

However it turns out that not all higher-order intertwining operators can be built by a ladder algorithm based on linear SUSY elements with real and nonsingular intermediate Hamiltonians.

\subsection{Generation of Polynomial SUSY QM by ladder: irreducible SUSY blocks of type I,} II, and III

Let us elucidate the circumstances which may obstruct the SUSY ladder decomposition of a Polynomial SUSY algebra. The class of Polynomial SUSY algebras can be extended admitting complex $\lambda_{l}$ and singular $h_{l}$. In fact, the full variety of building elements for non-linear SUSY can be established within the class of intertwining (or transformation) operators $q_{2}^{ \pm}$of second-order in derivatives. Eventually one has to find the basic set of Crum-Darboux operators (67) with nonsingular coefficient functions which produce a nonsingular potential $V^{(1)}$ after intertwining (66) with the Hamiltonian possessing a smooth initial potential $V^{(0)}$.

When taking a supercharge of second order in derivative with real coefficient functions one always can find formal (not necessarily normalizable) real zero-modes of the intertwining operators $q_{2}^{ \pm}$and, further on, the $2 \times 2$ matrix representation (75) for the super-Hamiltonian components $h^{(0)}, h^{(1)}$ by matrices $S^{ \pm}$. Then latter matrices can be obtained as real but, in general, not symmetric. Therefore the first obstruction for the ladder decomposition may arise when the reduction to a Jordan form does not yield real eigenvalues. For instance, if $h^{(0)} \phi_{i}^{-}(x)=\bar{\lambda} \epsilon_{i k} \phi_{k}^{-}(x)$ then the eigenvalues of 
$S^{+}=\bar{\lambda} \hat{\epsilon}$ are imaginary, mutually conjugated $\pm i \bar{\lambda}$. The possibility of complex pairs of mutually conjugated roots in a Polynomial SUSY algebra can be easily read off from its closure (76) as for supercharges with real coefficients polynomials $\mathcal{P}_{2}(H)$ possess real coefficients. We call this kind of irreducibility to be of type $I$. Its elementary block corresponds to the polynomial $\mathcal{P}_{2}^{(I)}(H)=(H+a)^{2}+d, \quad d>0$ and its analytical properties have been investigated in [47], [133]. Some examples of related isospectral potentials are described in [134].

Next, one has to ensure the positivity of the SUSY algebra relation (176) in a particular differential realization of a super-Hamiltonian $H$ with real non-singular potentials and the supercharges $Q_{N}^{+}, Q_{N}^{-}$(with $N=2$ in our case) made of differential operators with real coefficients. Let the energy spectrum $E_{j} ; j=0,1, \ldots ; E_{j}<E_{j+1}$ of $H$ be discrete, for simplicity. Then,

$$
\mathcal{P}_{N}\left(E_{j}\right)=\left\langle Q_{N}^{+} \Psi_{j} \mid Q_{N}^{+} \Psi_{j}\right\rangle+\left\langle Q_{N}^{-} \Psi_{j} \mid Q_{N}^{-} \Psi_{j}\right\rangle \geq 0,
$$

if the action of supercharges is well defined in the Hilbert space spanned on eigenfunctions of a super-Hamiltonian. It can be extended on a continuous energy spectrum with usage of wave packets.

Thus for non-singular potentials the acceptable disposition of polynomial roots related to zero-modes of a supercharge ensures non-negative values of $\mathcal{P}(E)$ for each energy level of a Hamiltonian. Accordingly, the following positions for polynomial real roots are acceptable(for a pair of complex, mutually conjugated roots the positivity is obvious) .

A. $\quad \lambda_{1} \leq \lambda_{2} \leq E_{0}$ or $\lambda_{1}=E_{0} ; \lambda_{2}=E_{1}$.

The related SUSY algebra is well embedded into a chain or ladder realization. It is reducible because one can gradually add/remove $\lambda_{1}$ and then $\lambda_{2}$ without breaking the positivity of intermediate SUSY algebra. The coincidence of roots and energies correspond to the isospectral transformation with deleting/inserting energy levels. For instance, if $\lambda_{1}=E_{0} ; \lambda_{2}=E_{1}$ then two pairs of zero-modes of $q_{2}^{ \pm}$can be chosen as solutions of Schrödinger equations with Hamiltonians $h^{(0)}, h^{(1)}$. One can implement 37] the energy levels $E_{0}, E_{1}$ to appear in any of the Hamiltonians $h^{(0)}, h^{(1)}$ but each level only once, either in $h^{(0)}$ or in $h^{(1)}$.

B. $E_{0}<\lambda_{1}<\lambda_{2} \leq E_{1}$ or $E_{j} \leq \lambda_{1}<\lambda_{2} \leq E_{j+1}, 1 \leq j$.

A pair of real roots is inserted between adjacent energy levels. Such an algebra cannot be decomposed into a chain of two linear SUSY with non-singular intermediate potentials as the removal of any of roots $\lambda_{1,2}$ immediately breaks the positivity in (87). Then the intermediate Hamiltonian acquires inevitably a singular potential which usage would lead to the loss of isospectrality. The related Darboux transformations had been known in 50ties [25]. We call this irreducibility to be of type II. The examples and certain theorems are given in [54], [134] (see also [77] for an exhaustive analysis of this type of irreducible Darboux transformations).

C. $E_{0}<\lambda_{1}=\lambda_{2} \leq E_{1}$ or $E_{j}<\lambda_{1}=\lambda_{2} \leq E_{j+1}, 1 \leq j$.

This is a confluent case which seems to be obtained as a limit of the previous one. 
However, one-dimensional QM does not allow degenerate levels and, besides, the matrix projection for the corresponding super-Hamiltonian contains a non-trivial Jordan cell. Therefore we specify this case as a separate one. This kind of irreducibility is named to be of type III. One may find more information on the analytical properties of related potentials in [135].

One can use these second-order blocks to build a $N$ th-order Polynomial SUSY system. Their general form is again given by Eq. (86) if to accept the presence of complex conjugated roots $\lambda_{l}$.

Meanwhile it seems that a pair of supercharge zero-modes or even a pair of new excited energy levels of the super-Hamiltonian can be always inserted by successive application of an appropriate ladder construction described in the previous Section using first-order intertwining transformations between regular Hamiltonians. But the order of a relevant ladder of first-order transformations and respectively of the final Polynomial SUSY will be certainly higher than two. We come to the problem of possible relationship between first-order reducible and irreducible SUSY algebras having the same superHamiltonian.

The related question concerns the degenerate roots. These roots are distributed between different Jordan cells in the matrices $S^{ \pm}$. The problem is on how many Jordan cells may coexist for the same eigenvalue and what is their role in the supercharge structure.

\subsection{Minimization of SUSY algebra for a given Hamiltonian and emergence of} irreducible blocks of type II and III

Let us reveal a possible redundancy in supercharges which can be eliminated without any changes in the super-Hamiltonian. There exists a possibility when the intertwining operators $q_{N}^{ \pm}$and $p_{N_{1}}^{ \pm}$for $N>N_{1}$ are related by a polynomial factor $F(x)$ depending on the Hamiltonian,

$$
q_{N}^{+}=F\left(h^{(0)}\right) p_{N_{1}}^{+}=p_{N_{1}}^{+} F\left(h^{(1)}\right) ; \quad q_{N}^{-}=F\left(h^{(1)}\right) p_{N_{1}}^{-}=p_{N_{1}}^{-} F\left(h^{(0)}\right) .
$$

Obviously in this case the reduction to the second supercharge does not result in any modifications of potentials.

Thus the problem consists in how to factorize a minimal essential part of the supercharge and avoid numerous SUSY algebras generated by means of "dressing" (88).

Let us illustrate how it could work in the following example:

the matrix $S^{-}$for the minimizable intertwining operator $k_{3}^{+}$with Jordan cells of different size having the same eigenvalue. It is generated by the operators,

$$
p^{ \pm}=\mp \partial+\chi, \quad h^{(1)}=p^{-} p^{+}+\lambda, \quad k_{3}^{+}=-p^{+} p^{-} p^{+}=p^{+}\left(\lambda-h^{(1)}\right) .
$$


Respectively, the basis of formal zero-modes (eigen- and associated functions) for the intertwining operator $k_{3}^{+} \phi_{j}=0$ generates the non-diagonal matrix $S^{-}$,

$$
\begin{array}{lclc}
\phi_{1}^{+}: & p^{-} p^{+} \phi_{1}^{+}=\phi_{2}^{+} & \longrightarrow & h^{(1)} \phi_{1}^{+}=\lambda \phi_{1}^{+}+\phi_{2}^{+} ; \\
\phi_{2}^{+}: & p^{+} \phi_{2}^{+}=0 & \longrightarrow & h^{(1)} \phi_{2}^{+}=\lambda \phi_{2}^{+} ; \\
\phi_{3}^{+}: & p^{+} \phi_{3}^{+} \neq 0, p^{-} p^{+} \phi_{3}^{+}=0 & \longrightarrow & h^{(1)} \phi_{3}^{+}=\lambda \phi_{3}^{+} ;
\end{array} \quad S^{-}=\left(\begin{array}{ccc}
\lambda & 1 & 0 \\
0 & \lambda & 0 \\
0 & 0 & \lambda
\end{array}\right) .
$$

Thus the algebraic redundancy in the operator $k_{3}^{+}$finds its unambiguous track in presence of two Jordan cells in the characteristic matrix $S^{-}$with the same eigenvalues.

The supercharge components cannot be factorized in the form (88) if the polynomial $\mathcal{P}_{N}(x)$ in the SUSY algebra closure (76) does not reveal the degenerate zeros. Indeed the SUSY algebra closure contains the square of polynomial $F(x)$, for instance,

$$
k_{N}^{-} k_{N}^{+}=F\left(h^{(1)}\right) p_{N_{1}}^{-} p_{N_{1}}^{+} F\left(h^{(1)}\right)=F^{2}\left(h^{(1)}\right) \mathcal{P}_{N_{1}}\left(h^{(1)}\right),
$$

where $\mathcal{P}_{N_{1}}(x)$ is a polynomial of lower order, $N_{1} \leq N-2$. Therefore each zero of the polynomial $F(x)$ will produce a double zero in the SUSY algebra provided by (91).

Thus the absence of double zeros is sufficient to deal with the SUSY charges nonfactorizable in the sense of Eq. (88). However it is not necessary because the degenerate zeros may well appear in the ladder construction giving new pairs of isospectral potentials (see, for instance, [47] for the Polynomial SUSY of second order).

Now we unravel the origin of irreducible, type-II and -III transformations based on the strip-off factorization. Let us consider an example of irreducible SUSY of type II with supercharges of second order in derivatives (see previous Subsection). Suppose that it realizes insertion of two new energy levels between the ground and first excited states. Then three lowest energy levels $E_{0}<E_{1}<E_{2}$ are of importance to study the relevant SUSY systems: the ground state level is degenerate between SUSY partners $h^{(0)}$ and $h^{(1)}$, i.e. $E_{0}^{(0)}=E_{0}^{(1)}$ whereas the two excited levels are present only in the spectrum of $h^{(1)}$.

Certainly one can use the ladder construction (80)-(84) to prepare the same spectral pattern. For this purpose, the intertwining operators (84) of forth order in derivatives must be employed. Indeed, one can prescribe the ladder steps for $q_{4}^{ \pm}$as follows: start from a pair of isospectral Hamiltonians with ground state energies $E_{3}$; generate the level $E_{0}$ in the Hamiltonian $h^{(0)}$ using the intertwining operators $r_{1}^{ \pm}$, then sequentially create in the spectrum of $h^{(1)}$ the state with energy $E_{2}<E_{3}$ by means of $r_{2}^{ \pm}$, next the energy level $E_{1}<E_{2}$ using $r_{3}^{ \pm}$and finally the ground state with energy $E_{0}<E_{1}$ exploiting $r_{4}^{ \pm}$. These elementary steps are reflected in zero-modes of the intertwining operators $q^{+}=r_{1}^{+} r_{2}^{+} r_{3}^{+} r_{4}^{+}$and $q^{-}=r_{4}^{-} r_{3}^{-} r_{2}^{-} r_{1}^{-}$. Namely the ground state of $h^{(0)}$ is a zero-mode of $r_{1}^{-}$(i.e. of $q^{-}$) whereas the states of $h^{(1)}$ for $E_{0}, E_{1}, E_{2}$ are annihilated by the product $r_{2}^{+} r_{3}^{+} r_{4}^{+}$(i.e. by $q^{+}$) according to Eq. (85)). As the ground state energies coincide for $h^{(0)}, h^{(1)}$ the Hamiltonian projections on the $q_{4}^{ \pm}$zero-mode space - the matrices $S^{ \pm}$ are, in general, not diagonalizable but have one rank-two Jordan cell each. Thus, for 
instance,

$$
S^{-}=\left(\begin{array}{cccc}
E_{0} & 0 & 0 & C \\
0 & E_{2} & 0 & 0 \\
0 & 0 & E_{1} & 0 \\
0 & 0 & 0 & E_{0}
\end{array}\right) \Longrightarrow \tilde{S}^{-}=\left(\begin{array}{cccc}
E_{0} & C & 0 & 0 \\
0 & E_{0} & 0 & 0 \\
0 & 0 & E_{2} & 0 \\
0 & 0 & 0 & E_{1}
\end{array}\right)
$$

where a non-zero constant $C$ can be normalized to $C=1$. The canonical Jordan form $\tilde{S}^{-}$in (92) is achieved by means of re-factorization $q^{+}=r_{1}^{+} r_{2}^{+} r_{3}^{+} r_{4}^{+}=r_{1}^{+} \tilde{r}_{2}^{+} \tilde{r}_{3}^{+} \tilde{r}_{4}^{+}$so that the generation of ground state for $h^{(0)}$ is associated now with $\tilde{r}_{2}^{ \pm}$. Respectively, the Polynomial SUSY algebra shows up one degenerate root,

$$
\mathcal{P}_{4}(H)=\left(H-E_{0}\right)^{2}\left(H-E_{1}\right)\left(H-E_{2}\right) .
$$

Evidently, this fourth-order algebra cannot be optimized to a lower-order one because there is no replication of roots in different Jordan cells of matrices $\tilde{\mathbf{S}}^{ \pm}$(see the "Stripoff" theorem on minimization in the next Section). However one may perform finetuning of Darboux transformation parameters to provide the constant $C=0$ in (92). This peculiar choice provides two rank-one cells in (92) with the same eigenvalue $E_{0}$. The SUSY algebra is still given by Eq. (93) but the intertwining operators reveal a redundancy,

$$
q_{4}^{+}=\left(h^{(0)}-E_{0}\right) q_{2}^{+} .
$$

By construction the left-hand side of this relation is fully factorizable in elementary binomials $r_{j}^{+}$with hermitian nonsingular intermediate Hamiltonians. But in the righthand side the operator $q_{2}^{+}=\tilde{r}_{3}^{+} \tilde{r}_{4}^{+}$does not admit a further factorization with a nonsingular intermediate Hamiltonian because after removal of the redundant factor $\left(h^{(0)}-E_{0}\right)$ such a factorization is forbidden by the positivity of the SUSY algebra, Eq. (87).

One can easily extrapolate the previous argumentation onto the case of additional degeneracy of excited levels $E_{1}=E_{2}$ to analyze the irreducible SUSY of type III. Thus we come to the conjectures that:

a) the factorization (84) of intertwining operators $q_{N}^{ \pm}$is not unique and there exist options to have more reducible ladders and less reducible ones with a larger number of singular intermediate Hamiltonians;

b) irreducible algebras of type II and III can be identified with special cases of fully reducible higher-order algebras of ladder type when the Hamiltonian projections $S^{ \pm}$ have an appropriate number of pairs of Jordan cells with coinciding eigenvalues.

We notice that one may save time in calculations when instead of a more lengthy binomial ladder construction the isospectral irreducible transformations of type II or III are exploited to embed pairs of energy levels between two excited ones. In the next section the results of a more rigorous investigation of the relationship between the reducible and irreducible intertwinings are presented. 


\section{Rigorous results on structure and (ir)reducibility of Polynomial SUSY}

Let us summarize the organization of Polynomial SUSY QM in a more systematic way. In this Section the single supercharge components are analyzed. In the general situation when the unique supercharge exists the minimization procedure is the way to factor out the redundant polynomials of Hamiltonian to leave the part essential for isospectral transformation of potentials after intertwining. In the consequent Sec. 5 devoted to hidden symmetries a more specific class of reflectionless potentials is involved and the two inequivalent supercharges are revealed. Then for lowering of their order as differential operators an additional procedure of optimization can be employed [73] which exploits linear combinations of different intertwining operators for a given pair of Hamiltonians with coefficients polynomial in Hamiltonians. Nevertheless, eventually the two supercharges of minimal order in derivatives survive after optimization and this very procedure is substantially based on the following theorems. We notice that the two theorems of subsec.5.1 are applicable to both non-periodic and periodic potentials whereas the two theorems of Subsec.5.2 have been proven for the special class of potentials (the Sokolov class) which does not include periodic potentials.

\subsection{Basic theorems on the structure of QM with a nonlinear SUSY}

First the following theorem regulates the very structure of Polynomial SUSY in terms of the Hamiltonian projection of the kernels of intertwining operators.

\section{Theorem 1 .}

Let $\phi_{n}^{\mp}(x), n=1, \ldots, N$ be a basis in $\operatorname{ker} q_{N}^{\mp}$ :

$$
q_{n}^{\mp} \phi_{n}^{\mp}=0, \quad q_{N}^{-}=\left(q_{N}^{+}\right)^{\dagger} .
$$

Then:

1) the action of the Hamiltonians $h^{(0)}, h^{(1)}$ on the functions $\phi_{n}^{ \pm}(x)$ is described by constant $N \times N$ matrices,

$$
h^{(0)} \phi_{n}^{-}=\sum_{m=1}^{N} S_{n m}^{+} \phi_{m}^{-} ; \quad h^{(1)} \phi_{n}^{+}=\sum_{m=1}^{N} S_{n m}^{-} \phi_{m}^{+} ; \quad n=1, \ldots, N ;
$$

2) the closure of the supersymmetry algebra takes a polynomial form,

$$
\left\{Q^{+}, Q^{-}\right\}=\operatorname{det}\left[E \mathbf{I}-S^{+}\right]_{E=H}=\operatorname{det}\left[E \mathbf{I}-S^{-}\right]_{E=H} \equiv \mathcal{P}_{N}(H),
$$

where $\mathbf{I}$ is an identity matrix and $S^{ \pm}$is the matrix with entries $S_{n m}^{ \pm}$.

Corollary 1. The spectra of the matrices $S^{+}$and $S^{-}$are equal.

Now we describe the minimization ("Strip-off") theorem.

A basis in the kernel of the intertwining operator in which the matrix $S$ of this operator has a Jordan form is called canonical; elements of a canonical basis are called transformation functions. 
The potentials $V^{(0)}(x)$ and $V^{(1)}(x)$ of the Hamiltonians $h^{(0)}$ and $h^{(1)}$ are interrelated by the equation $[28]$

$$
V^{(1)}(x)=V^{(0)}(x)-2[\ln W(x)]^{\prime \prime},
$$

where $W(x)$ is the Wronskian of elements of an arbitrary (a canonical as well) basis in $\operatorname{ker} q_{N}^{-}$.

The intertwining operators $q_{N}^{ \pm}$are called minimizable if these operators can be presented in the form

$$
q_{N}^{+}=\mathcal{P}\left(h^{(0)}\right) p_{M}^{+}=p_{M}^{+} \mathcal{P}\left(h^{(1)}\right), \quad q_{N}^{-}=\mathcal{P}\left(h^{(1)}\right) p_{M}^{-}=p_{M}^{-} \mathcal{P}\left(h^{(0)}\right),
$$

where $p_{M}^{ \pm}$are operators of order $M$ which intertwine the same Hamiltonians as $q_{N}^{ \pm}$and $\mathcal{P}(x)$ is a polynomial of degree $(N-M) / 2>0$. Otherwise the intertwining operators $q_{N}^{ \pm}$are named as non-minimizable.

The following theorem contains necessary and sufficient conditions under which an intertwining operator $q_{N}^{+}=\left(q_{N}^{+}\right)^{\dagger}$ is minimizable or not (a proof can be found in [73]).

("Strip-off") Theorem 2 on minimization of an intertwining operator $q_{N}^{+}$ (and equivalently $q_{2}^{-}$)

An intertwining operator $q_{N}^{+}$can be presented in the form

$$
q_{N}^{+}=p_{M}^{+} \prod_{l=1}^{m}\left(\lambda_{l}-h^{(1)}\right)^{\delta k_{l}},
$$

where $p_{M}^{+}$is a non-minimizable operator intertwining the same Hamiltonians as $q_{N}^{+}$(so that $p_{M}^{+} h^{(1)}=h^{(0)} p_{M}^{+}$), if and only if a Jordan form of the matrix $S^{+}$of the operator $q_{N}^{+}$ has $m$ pairs (and no more) of Jordan cells with equal eigenvalues $\lambda_{l}$ such that, for the $l$-th pair, $\delta k_{l}$ is an order of the smallest cell and $k_{l}+\delta k_{l}$ is an order of the largest cell . In this case, $M=N-2 \sum_{l=1}^{m} \delta k_{l}=\sum_{l=1}^{n} k_{l}$, where the $k_{l}, m+1 \leqslant l \leqslant n$ are orders of the remaining unpaired Jordan cells.

Remark 1. A Jordan form of the matrix $S^{+}$of the intertwining operator $q_{N}^{+}$cannot have more than two cells with the same eigenvalue $\lambda$; otherwise $\operatorname{ker}\left(\lambda-h^{(1)}\right)$ includes more than two linearly independent elements.

Corollary 2. Jordan forms of the matrices $S$ of the operators $q_{N}^{+}$and $q_{N}^{-}$coincide up to permutation of Jordan cells.

Remark 2. The Theorems 1 and 2 are valid for a broad class of potentials including the periodic ones.

If a Jordan form of the matrix $S$ of an intertwining operator has cells of order higher than one, then the corresponding canonical bases contains not only formal solutions of the Schrödinger equation but also formal associated functions, which are defined as follows [136].

A function $\psi_{n, i}(x)$ is called a formal associated function of $i$-th order of the Hamiltonian $h$ for a spectral value $\lambda_{n}$ if

$$
\left(h-\lambda_{n}\right)^{i+1} \psi_{n, i} \equiv 0, \quad \text { and } \quad\left(h-\lambda_{n}\right)^{i} \psi_{n, i} \not \equiv 0 .
$$


The term "formal" emphasizes that this function is not necessarily normalizable (not necessarily belongs to $L_{2}(\mathbb{R})$ ) and therefore it does not belong to the energy spectrum and, accordingly, is not included into the spectral resolution of identity. In particular, an associated function $\psi_{n, 0}$ of zero order is a formal eigenfunction of $h$ (not necessarily a normalizable solution of the homogeneous Schrödinger equation). They appear in the subspace of zero-mode solutions for irreducible intertwining operators and play role in building S-matrix of the Hamiltonian projection.

\subsection{Classification of really (ir)reducible SUSY transformations}

Assume that the intertwining operators $q_{N}^{ \pm}$are represented as products of the intertwining operators $k_{N-M}^{ \pm}$and $p_{M}^{ \pm}, 0<M<N$ so that

$q_{N}^{+}=p_{M}^{+} k_{N-M}^{+}, \quad q_{N}^{-}=k_{N-M}^{-} p_{M}^{-} ; \quad p_{M}^{+} h_{M}=h^{(0)} p_{M}^{+}, \quad p_{M}^{-} h^{(0)}=h_{M} p_{M}^{-} ;$

$k_{N-M}^{+} h^{(1)}=h_{M} k_{N-M}^{+}, \quad k_{N-M}^{-} h_{M}=h^{(1)} k_{N-M}^{-}, \quad h_{M}=-\partial^{2}+v_{M}(x)$,

where the coefficients $k_{N-M}^{ \pm}$and $p_{M}^{ \pm}$as well as the potential $v_{M}(x)$ may be complex and/or singular. The Hamiltonian $h_{M}$ is called intermediate with respect to $h^{(0)}$ and $h^{(1)}$. In this case, by Theorem 1, the spectrum of the matrix $S$ of the operator $q_{N}^{ \pm}$is a union of the spectra of the matrices $S$ for the operators $k_{N-M}^{ \pm}$and $p_{M}^{ \pm}$.

The intertwining operators $q_{N}^{ \pm}$are called reducible if these operators can be presented as products of two nonsingular intertwining operators (with real coefficients) $k_{N-M}^{ \pm}$and $p_{M}^{ \pm}, 0<M<N$ so that Eqs. (102) are valid and the intermediate Hamiltonian $h_{M}$ has a real nonsingular potential. Otherwise $q_{N}^{ \pm}$are called irreducible.

Irreducible, non-minimizable, intertwining operators of second order with real coefficients are divided into three types: [14].

An irreducible intertwining operator of I type is a differential intertwining operator with real coefficients for which eigenvalues of the matrix $S$ have nontrivial imaginary parts and are mutually complex conjugate.

An irreducible intertwining operator of the II type is a differential intertwining operator $q_{2}^{+}$(or equivalently $q_{2}^{-}$) of second order with real coefficients such that:

(1) eigenvalues of the matrix $S^{+}$of the operator $q_{2}^{+}$are real and different;

(2) both elements $\varphi_{1}^{+}(x)$ and $\varphi_{2}^{+}(x)$ of a canonical basis of $\operatorname{ker} q_{2}^{+}$have zeros.

An irreducible intertwining operator of the III type is a differential intertwining operator $q_{2}^{+}$(or equivalently $q_{2}^{-}$) of second order with real coefficients such that:

(1) the eigenvalues $\lambda_{1,2}$ of the matrix $S^{+}$of the operator $q_{2}^{+}$are equal, $\lambda_{1}=\lambda_{2}$;

(2) a canonical basis in $\operatorname{ker} q_{2}^{+}$consists of formal eigenfunctions, $\varphi_{10}^{+}(x)$, and associated functions, $\varphi_{11}^{+}(x)$, of the Hamiltonian $h^{(1)}$ which assemble into a Jordan cell,

$$
h^{(1)} \varphi_{10}^{+}=\lambda_{1} \varphi_{10}^{+}, \quad\left(h^{(1)}-\lambda_{1}\right) \varphi_{11}^{+}=\varphi_{10}^{+} ;
$$

(3) $\varphi_{10}^{+}(x)$ has at least one root.

Other types of irreducible non-minimizable intertwining operators of second order do not exist. 
Now let us state (in a concise form) two Theorems which characterize reducibility of intertwining operators of any order (proven in [75, 76, 77]):

Theorem 3. (on reducibility of "dressed" non-minimizable intertwining operators)

It asserts that for any non-minimizable intertwining operator with real spectrum of the matrix $S$, one can "dress" it, i.e. find and multiply by an appropriate polynomial of the Hamiltonian, so that the resulting (certainly minimizable) intertwining operator can be factorized into a product of intertwining operators solely of first order with intermediate real non-singular Hamiltonians. Thus any Polynomial SUSY algebra with real roots can be embedded into a higher order algebra corresponding to a ladder of linear SUSY.

Theorem 4. (on complete reducibility of non-minimizable intertwining operators)

It asserts that any non-minimizable intertwining operator with arbitrary spectrum of the matrix $S$ can be factorized into a product of intertwining operators of first order and irreducible second-order intertwining operators of the I, II and III type with intermediate real non-singular Hamiltonians. Still this factorization is not necessarily unique and, in particular, it may contain more or less blocks of first order.

These theorems have been formulated at full length and rigorously proven in [75, 76, 77] for the class $K$ (Sokolov class) of potentials $V(x)$ such that:

1) $V(x)$ is a real-valued function from $C_{\mathbb{R}}^{\infty}$;

2) there exist numbers $R_{0}>0, C>0$ (they depend on $V(x)$ ) such that the inequality $\inf _{|x| \geqslant R_{0}} V(x)+C>0$ takes place for any $|x| \geqslant R_{0}$;

3) the functions

$$
\left(\int_{ \pm R_{0}}^{x} \sqrt{\left|V\left(x_{1}\right)+C\right|} d x_{1}\right)^{2}\left(\frac{\left|V^{\prime}(x)\right|^{2}}{|V(x)+C|^{3}}+\frac{\left|V^{\prime \prime}(x)\right|}{|V(x)+C|^{2}}\right)
$$

are bounded for $x \geqslant R_{0}$ and $x \leqslant-R_{0}$, respectively. It can be proven [75] that the set $K$ is closed under intertwining of Hamiltonians.

The last condition is not very restrictive: for example, it is fulfilled (for $x \geq R_{0}$ ), for potentials:

1) $\quad V(x)=a x^{\gamma}[1+o(1)], \quad a>0, \quad \gamma>0$;

2) $\quad V(x)=V_{0}+a x^{-\gamma}[1+o(1)], \quad V_{0}>0, \quad a \in \mathbb{R}, \quad \gamma>0$;

3) $\quad V(x)=a x^{\alpha} e^{b x^{\beta}}[1+o(1)], \quad a>0, \quad b>0, \quad \alpha \in \mathbb{R}, \quad \beta>0$;

4) $\quad V(x)=V_{0}+a x^{\alpha} e^{-b x^{\beta}}[1+o(1)], \quad V_{0}>0, a \in \mathbb{R}, b>0, \alpha \in \mathbb{R}, \beta>0$.

A similar statement holds also for $x \leq-R_{0}$.

However periodic potentials do not belong to this class $K$. 


\section{Hidden symmetry in One-dimensional SUSY QM}

\subsection{Simplest SUSY with Hidden symmetry}

The emergence of hidden symmetries happens to be an intrinsic feature of SUSY algebra being related to so called central charges. Specifically in SUSY QM there are classes of potentials in super-Hamiltonians which structure is dictated by a hidden symmetry. In two (and more) dimensions the variety of such isospectral QM systems is rather typical and their spectra and eigenfunctions reveal partial or complete solvability accounted for by SUSY separation of variables (see Sections 8 and 9).

However hidden symmetries exist also in one-dimensional SUSY QM [78] if the same super-Hamiltonian conserves several non-trivial (non-minimizable and optimized[73]) supercharges. Following the algorithm of minimization on the variety of supercharges one can apply the optimization process[73] and prove that the maximal number of independent and non-minimizable (in the terminology of the previous Section) supercharges is two. Thus the two pairs of conserved supercharges $K^{+}, K^{-}$and $P^{+}, P^{-}$ may form two SUSY algebras for a hermitian Super-Hamiltonian $H$.

Let us examine the algebraic structure of the simplest non-linear SUSY with two non-minimizable supercharges,

$$
\begin{aligned}
& k^{ \pm} \equiv \partial^{2} \mp 2 f(x) \partial+\tilde{b}(x) \mp f^{\prime}(x) ; \\
& p^{ \pm} \equiv \mp \partial+\chi(x),
\end{aligned}
$$

induced by a complex supercharge of second order in derivatives (see the preprint version of [78] and [73]). The supersymmetries (121) generated by $K^{+}, K^{-}$and $P^{+}, P^{-}$prescribe that

$$
\begin{aligned}
& V^{(0),(1)}=\mp 2 f^{\prime}+f^{2}+\frac{f^{\prime \prime}}{2 f}-\left(\frac{f^{\prime}}{2 f}\right)^{2}-\frac{d}{4 f^{2}}-a, \\
& \tilde{b} \quad=f^{2}-\frac{f^{\prime \prime}}{2 f}+\left(\frac{f^{\prime}}{2 f}\right)^{2}+\frac{d}{4 f^{2}}, \\
& V^{(0),(1)}=\chi^{2} \mp \chi^{\prime},
\end{aligned}
$$

where $\chi, f$ are real functions and $a, d$ are real constants. The related superalgebra closure for $K^{+}, K^{-}$and $P^{+}, P^{-}$takes the form,

$$
\left\{K^{+}, K^{-}\right\}=(H+a)^{2}+d, \quad\left\{P^{+}, P^{-}\right\}=H .
$$

The compatibility of two supersymmetries is achieved by solutions of the following equations

$$
\chi=2 f, \quad f^{2}+\frac{f^{\prime \prime}}{2 f}-\left(\frac{f^{\prime}}{2 f}\right)^{2}-\frac{d}{4 f^{2}}-a=\chi^{2}=4 f^{2} .
$$

Eq. (108) represents a nonlinear second-order differential equation which solutions are parameterized by two integration constants. Therefore the existence of two SUSY reduces substantially the class of potentials for which they may appear. Evidently Eq. (108) can be integrated into the first-order one,

$$
\left(f^{\prime}\right)^{2}=4 f^{4}+4 a f^{2}+4 G_{0} f-d \equiv \Phi_{4}(f),
$$


where $G_{0}$ is a real constant.

The solutions of this equation are elliptic functions which can be found in the implicit form,

$$
\int_{f_{0}}^{f(x)} \frac{d f}{\sqrt{\Phi_{4}(f)}}= \pm\left(x-x_{0}\right)
$$

where $f_{0}$ and $x_{0}$ are real constants.

They are nonsingular if:

a) $\Phi_{4}(f)$ has three different real roots and the double root $\beta / 2$ is either the maximal one or a minimal one,

$$
\Phi_{4}(f)=4\left(f-\frac{\beta}{2}\right)^{2}\left(\left(f+\frac{\beta}{2}\right)^{2}-\left(\beta^{2}-\epsilon\right)\right), \quad 0<\epsilon<\beta^{2} .
$$

Then there exists a relation between constants $a, d, G_{0}$ in terms of coefficients $\beta, \epsilon$,

$$
a=\epsilon-\frac{3 \beta^{2}}{2}<0, \quad G_{0}=\beta\left(\beta^{2}-\epsilon\right), \quad d=\beta^{2}\left(\frac{3 \beta^{2}}{4}-\epsilon\right) .
$$

The constant $f_{0}$ is taken between the double root and a nearest simple root.

b) $\Phi_{4}(f)$ has two different real double roots which corresponds in (111), (112) to $G_{0}=0, \quad \beta^{2}=\epsilon>0, \quad a=-\epsilon / 2, \quad d=-\epsilon^{2} / 4$. The constant $f_{0}$ is taken between the roots.

The corresponding potentials $V^{(0)}, V^{(1)}$ are well known [137, 138] to be reflectionless, with one bound state at the energy $\left(\beta^{2}-\epsilon\right)$ and with the continuum spectrum starting from $\beta^{2}$. Respectively the scattering wave function is proportional to $\exp (i k x)$ with $k=\sqrt{E-\beta^{2}}$.

In the case a) the potentials coincide in their form and differ only by shift in the coordinate ("Darboux Displacement" [139]),

$$
V^{(0),(1)}=\beta^{2}-\frac{2 \epsilon}{\operatorname{ch}^{2}\left(\sqrt{\epsilon}\left(x-x_{0}^{(0),(1)}\right)\right)}, \quad x^{(0),(1)}=x_{0} \pm \frac{1}{4 \sqrt{\epsilon}} \ln \frac{\beta-\sqrt{\epsilon}}{\beta+\sqrt{\epsilon}},
$$

and in the case b) one of the potentials can be taken constant,

$$
V^{(0)}=\beta^{2}, \quad V^{(1)}=\beta^{2}\left(1-\frac{2}{\operatorname{ch}^{2}\left(\beta\left(x-x_{0}\right)\right)}\right),
$$

For these potentials one can elaborate the relations of extended SUSY algebra.

The initial algebra is given by the relations (107). It must be completed by the mixed anti-commutators containing symmetry operators,

$$
\left\{K^{+}, P^{-}\right\}=\left\{K^{-}, P^{+}\right\}^{\dagger}=\mathcal{B}(H)+\mathcal{E}(H),
$$

where the first term is Hermitian and in general (see the next Subsection) a polynomial of the super-Hamiltonian and the second one is anti-Hermitian and not a polynomial of $H$. In the example under consideration the first symmetry operator turns out to be constant, $\mathcal{B}(H)=G_{0}$ after taking into account (104) and (109). Meanwhile the second operator reads,

$$
\mathcal{E}(H)=\left[\mathbf{I} \partial^{3}-\left(a \mathbf{I}+\frac{3}{2} \mathbf{V}(x)\right) \partial-\frac{3}{4} \mathbf{V}^{\prime}(x)\right],
$$


in the notations $H \equiv-\partial^{2} \mathbf{I}+\mathbf{V}(x)$. By construction the operator $\mathcal{E}(H)$ realizes a new symmetry for the super-Hamiltonian. Directly from Eq. (116) one derives that,

$$
-\mathcal{E}^{2}(H)=H\left[(H+a)^{2}+d\right]-G_{0}^{2}=\left(H-E_{b}\right)^{2}\left(H-\beta^{2}\right),
$$

where $E_{b}=\beta^{2}-\epsilon$ is the energy of a bound state. Thus (some of) the zero modes of $\mathcal{E}(H)$ characterize either bound states or zero-energy states in the continuum. We remark that in the case b) only the Hamiltonian $h^{(1)}$ has a bound state. Accordingly the physical zero modes of $\mathcal{E}(H)$ may be either degenerate [case a), broken SUSY] or non-degenerate [case b), unbroken SUSY].

The square root in (117) can be established from the analysis of scattering (transmission) coefficients,

$$
\mathcal{E}(H)=i\left(E_{b}-H\right) \sqrt{H-\beta^{2}} .
$$

An unambiguous determination of this square root needs to specify the space of asymptotic "incoming" states on which this operator acts, i.e. the scattering condition which selects out the direction of scattering either from the left to the right or in the opposite direction. These two spaces do not overlap. In (118) the first type of scattering condition has been selected: from the left to the right with the asymptotic limit of an incoming state, $x \rightarrow-\infty, \Psi(k, x) \rightarrow \exp (i k x), k>0$. For the alternative selection one should change the sign of square root. We notice that the symmetry operator (116), (118) is irreducible, i.e. the binomial $\left(E_{b}-H\right)$ cannot be removed. Indeed the elimination of this binomial would lead to an essentially nonlocal operator.

When taking Eq. (118) into account one finds (on the same space of scattering states) the mixed anti-commutators of the extended SUSY algebra (115) in a nonpolynomial form,

$$
\left\{K^{+}, P^{-}\right\}=\left\{K^{-}, P^{+}\right\}^{\dagger}=G_{0}-i\left(H-E_{b}\right) \sqrt{H-\beta^{2}} .
$$

Thus the "central charge" of the extended SUSY is built of the elements (107) and (119) and cannot be diagonalized by a unitary rotation with elements polynomial in $H$. Therefore the SUSY algebra is extended in the class of differential operators of finite order. The existence of polynomial relations between commuting operators can be in fact established from the Burchnall-Chaundy theorem [140] as it was noticed recently in [141.

\subsection{General case: one-dim SUSY algebra with Hidden symmetry}

Let us examine the very possibility to have several supercharges for the same superHamiltonian. We remind that a number of supercharges can be produced trivially with the help of multiplication on a polynomial of the Hamiltonian (see Section 4). Certainly such supersymmetries are absolutely equivalent for the purposes of spectral design and one must get rid of them. It was proved in [73] that the infinite set of possible supercharges for a given super-Hamiltonian can be always optimized so that no more than two nontrivial supercharges remain and that an optimal set of two real 
supercharges $K^{ \pm}$and $P^{ \pm}$with components of minimal order in derivatives contains one operator of odd-order in derivatives and another one of even-order. They can be used to generate all possible conserved supercharges $Q_{i}$ by "dressing" their components with polynomials of the Hamiltonians,

$$
q_{i}^{ \pm}=\alpha_{i}^{ \pm} k^{ \pm}+\beta_{i}^{ \pm} p^{ \pm} .
$$

Thus in one-dimensional (scalar) QM one may have the $\mathcal{N}=1,2$ SUSY only.

Correspondingly suppose that the super-Hamiltonian $H$ admits two supersymmetries with supercharges $K^{ \pm}$and $P^{ \pm}$made of differential intertwining operators of order $N$ and $N_{1}$ respectively,

$$
\left[H, K^{ \pm}\right]=\left[H, P^{ \pm}\right]=0 .
$$

The second pair of supercharges $P^{ \pm}$is assumed to be made of differential operators of lower order $N_{1}<N$.

To close the algebra one has to include all anti-commutators between supercharges, i.e. the full algebra based on two pairs of supercharges $K^{ \pm}$and $P^{ \pm}$with real intertwining operators. Two supercharges generate two Polynomial SUSY,

$$
\left\{K^{+}, K^{-}\right\}=\tilde{\mathcal{P}}_{N}(H), \quad\left\{P^{+}, P^{-}\right\}=\tilde{\mathcal{P}}_{N_{1}}(H) .
$$

The closure of the extended, $\mathcal{N}=2$ SUSY algebra is given by

$$
\begin{gathered}
\left\{P^{-}, K^{+}\right\} \equiv \mathcal{R}=\left(\begin{array}{cc}
p_{N_{1}}^{+} k_{N}^{-} & 0 \\
0 & k_{N}^{-} p_{N_{1}}^{+}
\end{array}\right), \\
\left\{K^{-}, P^{+}\right\} \equiv \mathcal{R}^{\dagger}=\left(\begin{array}{cc}
k_{N}^{+} p_{N_{1}}^{-} & 0 \\
0 & p_{N_{1}}^{-} k_{N}^{+}
\end{array}\right) .
\end{gathered}
$$

Evidently the components of operators $\mathcal{R}, \mathcal{R}^{\dagger}$ are differential operators of $N+N_{1}$ order commuting with the Hamiltonians $h^{(0)}, h^{(1)}$, hence they form symmetry operators $\mathcal{R}, \mathcal{R}^{\dagger}$ for the super-Hamiltonian. However, in general, they are not polynomials of the Hamiltonians $h^{ \pm}$and these symmetries impose certain constraints on potentials.

All four operators $\tilde{\mathcal{P}}_{N}(H), \tilde{\mathcal{P}}_{N_{1}}(H), \mathcal{R}, \mathcal{R}^{\dagger}$ commute each to other. The Hermitian matrix characterizing this $\mathcal{N}=2$ SUSY,

$$
\mathcal{Z}(H)=\left(\begin{array}{cc}
\tilde{\mathcal{P}}_{N}(H) & \mathcal{R} \\
\mathcal{R}^{\dagger} & \tilde{\mathcal{P}}_{N_{1}}(H)
\end{array}\right), \quad \operatorname{det}[\mathcal{Z}(H)]=\tilde{\mathcal{P}}_{N} \tilde{\mathcal{P}}_{N_{1}}-\mathcal{R} \mathcal{R}^{\dagger}=0,
$$

is degenerate. Therefore it seems that the two supercharges are not independent and by their redefinition (unitary rotation) one might reduce the extended SUSY to an ordinary $\mathcal{N}=1$ one. However such rotations cannot be global (constant) and must use non-polynomial, pseudo-differential operators as "parameters". Indeed, the operator components of the "central charge" matrix $\mathcal{Z}(H)$ have different order in derivatives. Thus, globally the extended nonlinear SUSY deals with two sets of supercharges but when they act on a given eigenfunction of the super-Hamiltonian $H$ one could, in principle, perform the energy-dependent rotation and eliminate a pair of supercharges. 
Nevertheless this reduction can be possible only after the constraints on potentials have been resolved.

Let us find the formal relation between the symmetry operators $\mathcal{R}, \mathcal{R}^{\dagger}$ and the super-Hamiltonian. These operators can be decomposed into a hermitian and an antihermitian parts,

$$
\mathcal{B} \equiv \frac{1}{2}\left(\mathcal{R}+\mathcal{R}^{\dagger}\right) \equiv\left(\begin{array}{cc}
b^{+} & 0 \\
0 & b^{-}
\end{array}\right), \quad \mathcal{E} \equiv \frac{1}{2}\left(\mathcal{R}^{\dagger}-\mathcal{R}\right) \equiv\left(\begin{array}{cc}
e^{+} & 0 \\
0 & e^{-}
\end{array}\right) .
$$

The operator $\mathcal{B}$ is a differential operator of even order and therefore it may be a polynomial of the super-Hamiltonian $H$. But if the operator $\mathcal{E}$ does not vanish identically it is a differential operator of odd order and cannot be realized by a polynomial of $H$.

It can be proven [73] that the hermitian operator $\mathcal{B}$ is indeed a polynomial of the super-Hamiltonian of the order $N_{b} \leq N-1$. Let us use it to unravel the superHamiltonian content of the operator $\mathcal{E}$,

$$
\mathcal{E}^{2}(H)=\tilde{\mathcal{P}}_{N}(H) \tilde{\mathcal{P}}_{N_{1}}(H)-\mathcal{B}^{2}(H),
$$

which follows directly from (124) and (125). As the (nontrivial) operator $\mathcal{E}(H)$ is a differential operator of odd order $N_{e}$ it may have only a realization non-polynomial in $H$ being a square root of (126) in an operator sense. An unambiguous determination of this square root needs to specify the space of asymptotic "incoming" states on which this operator acts, i.e. the scattering condition (see the previous Subsection). The symmetry operator is certainly non-trivial if the sum of orders $N+N_{1}$ of the operators $k_{N}^{ \pm}$and $p_{N_{1}}^{ \pm}$is odd and therefore $N_{e}=N+N_{1}$.

The existence of a nontrivial symmetry operator $\mathcal{E}$ commuting with the superHamiltonian results in common eigenstates which however are not necessarily physical wave functions. In general they may be combinations of two solutions of the Shrödinger equation with a given energy, the physical and unphysical ones. But if the symmetry operator $\mathcal{E}$ is anti-Hermitian in the Hilbert space spanned on the eigenfunctions of the super-Hamiltonian $H$ then both operators have a common set of physical wave functions. This fact imposes quite rigid conditions on partner potentials $V^{(0),(1)}$. It can be proven [73] that they inevitably belong to the class of transparent or reflectionless ones [137, 138]. Such a symmetry has relations to the Lax method in the soliton theory [28, 29].

As the symmetry operator $\mathcal{E}$ is anti-Hermitian its eigenvalues are imaginary but, by construction, its coefficients are real. Since the wave functions of bound states of the system $H$ can always be chosen to be real we conclude that they must be zero-modes of the operator $\mathcal{E}(H)$,

$$
\mathcal{E}(H) \psi_{i}=\mathcal{E}\left(E_{i}\right) \psi_{i}=0, \quad \tilde{\mathcal{P}}_{N}\left(E_{i}\right) \tilde{\mathcal{P}}_{N_{1}}\left(E_{i}\right)-\mathcal{B}^{2}\left(E_{i}\right)=0,
$$

which represents the algebraic equation on bound state energies of a system possessing two supersymmetries. Among solutions of (127) one reveals also a zero-energy state at the bottom of continuum spectrum. 


\section{Shape invariance for SUSY related potentials}

\subsection{Shape invariance in linear SUSY}

One of the goal of SUSY QM design consists in search for exactly solvable models of Quantum Mechanics. For one-dimensional Schrödinger equation, a list of exactly solvable is well known: harmonic oscillator, Coulomb, Morse, Pöschl-Teller, Scarf, Eckart potentials etc 142. Each of such potentials has its own history of solution, but all of them were reproduced in the framework of a single algebraic procedure of Factorization Method [35] in the middle of last century. The related method was formulated in the context of modern Supersymmetric Quantum Mechanics with the help of new notion - shape invariance - introduced by L.E.Gendenshtein in [45].

The basic steps of standard shape invariance in absence of spontaneous SUSY breaking [45], [143] - [145], 4] are the following. Let us consider a parametric family of one-dimensional superpartners $h^{(0)}(a), h^{(1)}(a)$ and first order supercharges $q^{ \pm}(a)$ depending on a parameter $a$. We say that the Hamiltonians are shape invariant when in addition to supersymmetrical intertwining relations (44) they have the property (analogous to "cyclic" in [123, 125]),

$$
h^{(1)}(a)=h^{(0)}(\bar{a})+\mathcal{R}(a),
$$

where $\bar{a}=\bar{a}(a)$ is a modified value of parameter depending on $a$ and $\mathcal{R}(a)$ is a (cnumber) function of $a$ taken positive for definiteness. Then the absence of spontaneous breaking of supersymmetry for all values of $a$ implies that just the lowest eigenvalue $E_{0}(a)$ of $h^{(0)}(a)$ vanishes, and the corresponding eigenfunctions $\Psi_{0}^{(0)}(a)$ are normalizable zero modes of $q^{-}(a)$. This property allows to solve the models (i.e. to find the spectrum and all bound state wave functions) algebraically.

To perform it we start from

$$
h^{(0)}(\bar{a}) \Psi_{0}^{(0)}(\bar{a})=E_{0}(\bar{a}) \Psi_{0}^{(0)}(\bar{a})=0 .
$$

Consider the relation (128) to obtain,

$$
h^{(1)}(a) \Psi_{0}^{(0)}(\bar{a})=\mathcal{R}(a) \Psi_{0}^{(0)}(\bar{a}) .
$$

We notice that $\Psi_{0}^{(0)}(\bar{a}) \equiv \Psi_{0}^{(1)}(a)$ has no nodes and therefore is the ground state wave function of $h^{(1)}(a)$. The combination of (4) and (130) yields,

$$
h^{(0)}(a) q^{+}(a) \Psi_{0}^{(0)}(\bar{a})=\mathcal{R}(a) q^{+}(a) \Psi_{0}^{(0)}(\bar{a}) .
$$

Provided $q^{+}(a) \Psi_{0}^{(0)}(\bar{a})$ is normalizable, we have generated an excited state of $h^{(0)}(a)$. These steps can be repeated up to the last step, where the resulting wave function $\Psi$ will not be normalizable anymore. There are notorious cases (oscillator-like potentials) where the spectrum is not bounded from above. The simplest and famous case is the harmonic oscillator with $q^{ \pm}=\mp \partial+\lambda x$ and $\mathcal{R}(a)=2 \lambda$. By the cyclic algorithm one easily reconstructs the energy spectrum $E_{n}^{(0)}=2 n \lambda$.

It is clear that the isospectrality of $h^{(0)}(a)$ and $h^{(1)}(a)$ (up to the only zero mode $\left.\Psi_{0}^{(0)}(a)\right)$ implies that there is no eigenvalue of $h^{(0)}(a)$ between zero and the ground state 
energy $E_{0}^{(1)}(a)$ of $h^{(1)}$. This observation can be used to prove that after a number of iterations one gets the entire spectrum of $h^{(0)}(a)$. Thus the shape invariance or cyclic method leads to spectrum generating algebras which allow to find algebraically all energy eigenvalues and eigenfunctions of shape invariant Hamiltonians in one-dimensional SUSY QM - complete solvability.

We notice that the shape invariance relation (128) combined with SUSY factorization of Hamiltonians (1), (3) entails the conventional Heisenberg algebra,

$$
\left[q^{-}, q^{+}\right]=\mathcal{R}(a),
$$

to supply $q^{ \pm}$with the meaning of creation and annihilation operators.

\subsection{Intertwining with shift and higher order shape-invariance}

The particular construction of higher order shape-invariance can be realized [78] by breaking of hidden symmetry (121) for two Polynomial SUSY algebras (122),

$h^{(0)} k_{N}^{+}=k_{N}^{+} h^{(1)} ; \quad k_{N}^{+} k_{N}^{-}=\tilde{\mathcal{P}}_{N}\left(h^{(0)}\right) ; \quad k_{N}^{-} k_{N}^{+}=\tilde{\mathcal{P}}_{N}\left(h^{(1)}\right) ;$

$h^{(0)} p_{N_{1}}^{+}=p_{N_{1}}^{+}\left(h^{(1)}+2 \lambda\right) ; \quad p_{N_{1}}^{+} p_{N_{1}}^{-}=\tilde{\mathcal{P}}_{N_{1}}\left(h^{(0)}\right) ; \quad p_{N_{1}}^{-} p_{N_{1}}^{+}=\tilde{\mathcal{P}}_{N_{1}}\left(h^{(1)}+2 \lambda\right) ;$

with the help of shift on a positive constant $\mathcal{R}(a) \equiv 2 \lambda$. In the sector of one of partner Hamiltonians (for example, $h^{(0)}$ ) we obtain,

$$
h^{(0)} \mathbf{a}^{+}=\mathbf{a}^{+}\left(h^{(0)}+2 \lambda\right), \quad \mathbf{a}^{-} h^{(0)}=\left(h^{(0)}+2 \lambda\right) \mathbf{a}^{-}
$$

if introducing the product operators $\mathbf{a}^{+} \equiv p_{N_{1}}^{+} k_{N}^{-}=\left(\mathbf{a}^{-}\right)^{\dagger}$. We will call such a Hamiltonian as "higher order shape-invariant". One can also work the other way around and start from (135) with a differential operator $\mathbf{a}^{+}$of $N_{1}+N$ order in derivatives, factorize the latter as a product $p_{N_{1}}^{+} k_{N}^{-}$with nonsingular operator multipliers and find an auxiliary super-partner Hamiltonian $h^{(1)}$ to obtain (133).

Eq.(135) is a spectrum generating, ladder ("dressing chain" [123, 125]) equation where $\mathbf{a}^{+}$plays the role of generalized creation operator which provides an excitation energy of $2 \lambda$. In order to study the spectrum it is essential to determine normalizable zero modes of $\mathbf{a}^{-}$and $\mathbf{a}^{+}$. The former ones describe the lowest lying levels of the system for positive $2 \lambda$ and one has to apply recursively the operator $\mathbf{a}^{+}$to them in order to generate the excitation spectrum. The energies of the zero modes can be obtained from vanishing the average of the operator product $\mathbf{a}^{+} \mathbf{a}^{-}$. In Nonlinear (Polynomial) SUSY this product can be evaluated algebraically because [47.

$$
\mathbf{a}^{+} \mathbf{a}^{-}=p_{N_{1}}^{+} k_{N}^{-} k_{N}^{+} p_{N_{1}}^{-}=p_{N_{1}}^{+} \tilde{\mathcal{P}}_{N}\left(h^{(1)}\right) p_{N_{1}}^{-}=\tilde{\mathcal{P}}_{N}\left(h^{(0)}-2 \lambda\right) \tilde{\mathcal{P}}_{N_{1}}\left(h^{(0)}\right),
$$

see (122) . In contrast to the simple harmonic oscillator, one has also the possibility of zero modes of the operator $\mathbf{a}^{+}$. Accordingly in this case the relevant operator product reads

$$
\mathbf{a}^{-} \mathbf{a}^{+}=\tilde{\mathcal{P}}_{N}\left(h^{(0)}\right) \tilde{\mathcal{P}}_{N_{1}}\left(h^{(0)}+2 \lambda\right),
$$

where the polynomials contain zeros some of which correspond to zero modes of the operator $\mathbf{a}^{+}$. Thus (135) provides a connection between different levels of $h^{(0)}$ by a given 
shift, which is the simplest realization of the notion of shape-invariance [45]. Though some properties of the spectrum, like normalizable zero modes of $\mathbf{a}^{ \pm}$, will depend on the explicit product structure of $\mathbf{a}^{ \pm}$, the equidistant excitation spectrum and corresponding wave functions can be mainly obtained algebraically .

The generalized (or deformed) Heisenberg algebra is finally built by the following closure,

$$
\left[\mathbf{a}^{+}, \mathbf{a}^{-}\right]=\tilde{\mathcal{P}}_{N}\left(h^{(0)}-2 \lambda\right) \tilde{\mathcal{P}}_{N_{1}}\left(h^{(0)}\right)-\tilde{\mathcal{P}}_{N}\left(h^{(0)}\right) \tilde{\mathcal{P}}_{N_{1}}\left(h^{(0)}+2 \lambda\right) \sim 2 \lambda \mathcal{P}_{N+N_{1}-1}\left(h^{(0)}\right) .
$$

It is possible to study the certain consequences of (135) algebraically, using the deformed Heisenberg algebra (138) without taking into account the specific definition of the operators $\mathbf{a}^{ \pm}$although the physical spectrum is essentially based on normalizable zero modes of $\mathbf{a}^{ \pm}$.

The important comment is in due. Taking into account the minimization recipe from Subsection 3.4 and the Theorem 2 from Subsection 4.1 one can find in certain cases that the order of operators $p_{N_{1}}^{ \pm}$and $k_{N}^{ \pm}$can be lowered without changing the potential in the Hamiltonian $h^{(0)}$. However the appropriate factorization of redundant polynomials of the Hamiltonian can be typically realized only at the level of individual SUSY algebras and, in general, cannot be extended to the deformed Heisenberg algebra (138). Thus at the formal level there may be several algebras (138) with the same spectrum pattern.

\subsection{Intertwining with shift and second order shape-invariance}

Let's elucidate the particular case when $\mathbf{a}^{ \pm}$is of second order, in which we can obtain a generalized singular harmonic oscillator which is also shape-invariant. Correspondingly we consider in (133), (134) $k^{ \pm}, p^{ \pm}$of first order. We can thereby explore that they can lead to nontrivial consequences. The superpotentials which solve (133), (134) are growing linearly for large $x$ and behave like $1 / x$ at the origin just leading to two singular potentials [17] $V^{(0)}(x)$ and $V^{(1)}(x)$ as follows,

$$
V^{(0)}(x)=\frac{\rho(\rho-1)}{x^{2}}+\frac{\lambda^{2} x^{2}}{4}+\lambda\left(\rho+\frac{1}{2}\right) ; \quad V^{(1)}(x)=\frac{\rho(\rho+1)}{x^{2}}+\lambda^{2} x^{2}+\lambda\left(\rho-\frac{1}{2}\right) \cdot(139
$$

These potentials are shape-invariant in the standard sense [45] and belong to the class of algebraically solvable models, because $V^{(1)}(x ; \rho, \lambda)=V^{(0)}(x ; \rho+1, \lambda)-2 \lambda$. On the half-line with suitable boundary conditions for wave functions [47] at the origin the above potentials can be interpreted as radial harmonic oscillators for integer values of $\rho$. However this system makes sense also for arbitrary real $\rho$.

The algebraic properties of these systems are based on the following factorization,

$h^{(0)}=k^{+} k^{-}=p^{+} p^{-}+\lambda(2 \rho+1) ; \quad h^{(1)}=k^{-} k^{+}=p^{-} p^{+}+\lambda(2 \rho-1)$,

satisfying the relations (133), (134) . The intertwining operators are given by,

$$
k^{+}=\left(k^{-}\right)^{\dagger}=-\partial-\frac{\rho}{x}-\frac{\lambda x}{2} ; \quad p^{+}=-\partial-\frac{\rho}{x}+\frac{\lambda x}{2} .
$$


In terms of the product operators $\mathbf{a}^{+} \equiv p^{+} k^{-}$and $\mathbf{a}^{-} \equiv k^{+} p^{-}$one obtains an algebra suggestive of a generalization of the standard harmonic oscillator algebra,

$$
\left[h^{(0)}, \mathbf{a}^{ \pm}\right]= \pm 2 \lambda \mathbf{a}^{ \pm}
$$

where the raising/lowering ("creation/annihilation") operators $\mathbf{a}^{ \pm}$can be presented in the form convenient for further evaluations,

$$
\mathbf{a}^{+}=\left(\mathbf{a}^{-}\right)^{\dagger}=\exp \left\{\frac{\lambda x^{2}}{4}\right\}\left(-\partial^{2}+\frac{\rho(\rho-1)}{x^{2}}\right) \exp \left\{-\frac{\lambda x^{2}}{4}\right\} .
$$

Similar results hold for $h^{(1)}$.

Meanwhile the crucial polynomial algebra describes the individual products,

$\mathbf{a}^{+} \mathbf{a}^{-}=\left(h^{(0)}-2 \lambda\right)\left(h^{(0)}-\lambda(2 \rho+1)\right) ; \quad \mathbf{a}^{-} \mathbf{a}^{+}=h^{(0)}\left(h^{(0)}-\lambda(2 \rho-1)\right)$.

The zeros of polynomials in (144) may indicate the zero modes ("vacuum states") of the annihilation, $\mathbf{a}^{-}$or creation, $\mathbf{a}^{+}$operators. In the case under discussion it happens that the appropriate normalizable zero modes appear for the operators $k^{+}$and $p^{-}$,

$k^{+} \psi_{0,1}=0, \quad \psi_{0,1}=x^{-\rho} \exp \left\{-\frac{\lambda x^{2}}{4}\right\} ; \quad p^{-} \tilde{\psi}_{0,2}=0, \quad \tilde{\psi}_{0,2}=x^{\rho} \exp \left\{-\frac{\lambda x^{2}}{4}\right\}$,

which entails the two zero modes for the annihilation operator,

$\mathbf{a}^{-} \psi_{0, i}=0 ; \quad \psi_{0,1}=x^{-\rho} \exp \left\{-\frac{\lambda x^{2}}{4}\right\} ; \quad \psi_{0,2}=x^{1-\rho} \exp \left\{-\frac{\lambda x^{2}}{4}\right\}$.

Accordingly they are associated to two zeros of the first polynomial in (144),

$$
\left(h^{(0)}-2 \lambda\right) \psi_{0,1}=0=\left(h^{(0)}-\lambda(2 \rho+1)\right) \psi_{0,2} .
$$

Another couple of zeros for the second polynomial in (144) are not related to any normalizable solution.

Eventually the oscillator algebra (142) generates one ladder of equidistant levels for $\rho \geq \frac{3}{2}$ or, equivalently for $\rho \leq-\frac{1}{2}$ and two independent ladders of equidistant levels for $-\frac{1}{2}<\rho<\frac{1}{2}$ or, equivalently, $\frac{1}{2}<\rho<\frac{3}{2}$. For $\rho=\frac{1}{2}$ these two ladders coincide. The corresponding spectra of $h^{(0)}$ are: $E_{n, 1}=2 \lambda(n+1)$ or $E_{n, 2}=\lambda(2 \rho+1+2 n)$ for $n=0,1, \ldots$

The generalized Heisenberg algebra for this sort of oscillator reads,

$$
\left[\mathbf{a}^{+}, \mathbf{a}^{-}\right]=-4 \lambda h^{(0)}+2 \lambda^{2}(2 \rho+1) .
$$

There might be an (superficial) impression of the algebra (142) as to be a broken hidden symmetry of the $\mathcal{N}=2$ SUSY (122), (123). However the contraction $\lambda \rightarrow 0$ results in the system with $\mathbf{a}^{ \pm} \sim h^{(0)}$ and continuum spectrum and therefore no any nontrivial hidden symmetry emerges. One can find the deformed Heisenberg algebra (148) arising in a more complicated setting of superconformal mechanics [146].

A nontrivial, shape-invariant, breaking of the $\mathcal{N}=2$ SUSY arises for the third-order spectrum-generating algebra. 


\subsection{Intertwining with shift and third order shape-invariance}

Let us now consider the intertwining operator $k^{ \pm}$to be a reducible or irreducible operator of second order but $p^{ \pm}$still of first order like in (104) [78], 80]. Accordingly they are parameterized by

$$
\begin{aligned}
& k^{ \pm} \equiv \partial^{2} \mp 2 f(x) \partial+\tilde{b}(x) \mp f^{\prime}(x) ; \\
& p^{ \pm}=\mp \partial+\chi(x) .
\end{aligned}
$$

The solutions of (133) are unchanged in respect to Eqs.(105) of Subsection 5.1 but Eq.(134) implies a shift for the potential $V^{(1)}(x)$ in (106). Thus the consistency equations for $\chi(x)$ and $f(x)$ are modified. The first Eq.(108) becomes:

$$
\chi(x)=2 f(x)+\lambda x,
$$

where a possible integration constant can be ignored because of a shift of $x$, which fixes the origin of coordinate. Then the potential $V^{(0)}(x)$ can be written from (105) as:

$$
V^{(0)}(x)=-2 f^{\prime}(x)+4 f^{2}(x)+4 \lambda x f(x)+\lambda^{2} x^{2}-\lambda .
$$

The coefficient in the intertwining operator $k^{ \pm}$reads,

$$
\tilde{b}=-\left(2 f^{2}+4 \lambda x f+4 \lambda^{2} x^{2}+a\right) .
$$

In the second Eq. (108) one has to replace $\chi^{2}$ in accordance with (151), i.e. $f(x)$ satisfies the following equation,

$$
f^{\prime \prime}=\frac{f^{\prime 2}(x)}{2 f(x)}+6 f^{3}(x)+8 \lambda x f^{2}(x)+2\left(\lambda^{2} x^{2}-\lambda+a\right) f(x)+\frac{d}{2 f(x)} .
$$

The equation (154) can be transformed by the substitution $f(x) \equiv 1 / 2 \sqrt{\lambda} g(y) ; y \equiv \sqrt{\lambda} x$ to the Painleve-IV equation [79],

$$
g^{\prime \prime}=\frac{g^{\prime 2}(y)}{2 g(y)}+\frac{3}{2} g^{3}(y)+4 y g^{2}(y)+2\left(y^{2}-\alpha\right) g(y)-\frac{b}{2 g(y)} .
$$

where

$$
\alpha \equiv 1-\frac{a}{\lambda} ; \quad b \equiv-\frac{4 d}{\lambda^{2}} .
$$

This equation has been studied intensively in the last years [81, [82].In what follows we will focus mainly on asymptotic properties of its solutions which will determine the asymptotics of potentials (152) and the normalizability of eigenfunctions.

The spectrum generating operators $\mathbf{a}^{+}=p^{+} k^{-}=\left(\mathbf{a}^{-}\right)^{\dagger}$ form the polynomial algebra,

$$
\mathbf{a}^{+} \mathbf{a}^{-}=h^{(0)}\left[\left(h^{(0)}+a-2 \lambda\right)^{2}+d\right] ; \quad \mathbf{a}^{-} \mathbf{a}^{+}=\left[h^{(0)}+2 \lambda\right]\left[\left(h^{(0)}+a\right)^{2}+d\right] .
$$

We show how one can derive the spectrum from (135) and (160) if normalizable zero modes of the annihilation operator $\mathbf{a}^{-}$exist. We stress that this algebraic method is very powerful now since the explicit form of the potential is known only in terms of Painleve transcendents. 
The equation for zero modes of $\mathbf{a}^{-}$reads:

$$
\mathbf{a}^{-} \Psi_{k}^{(0)}=k^{+} p^{-} \Psi_{k}^{(0)}=0
$$

where $k$ labels the normalizable solutions.

In accordance to the non-linear SUSY algebra (157), the equation for eigenvalues $E_{k}^{(0)}$ is,

$$
E^{(0)} \cdot\left[\left(E^{(0)}+a-2 \lambda\right)^{2}+d\right]=0,
$$

and has at most three real solutions.

Concerning the generalized Heisenberg algebra [83], the modification in respect to (142) in the previous Subsection is given by,

$$
\left[\mathbf{a}^{+}, \mathbf{a}^{-}\right]=-2 \lambda\left(3\left(h^{(0)}\right)^{2}+(4 a-2 \lambda) h^{(0)}+a^{2}+d\right)
$$

and similarly for $h^{(1)}$.

\subsection{Spectrum patterns for third order shape-invariance}

We give now a short description of typical spectrum patterns (the details can be found in [78]), [80]. Below on, the case of equal asymptotics of $f(x)$ at $\pm \infty$ is discussed.

- Three normalizable zero modes of $\mathbf{a}^{-}$and, respectively, three equidistant sequences of levels may arise only for $\lambda>\sqrt{-d}$ if $-\sqrt{-d}>a>\sqrt{-d}-2 \lambda$ or $2 \lambda-\sqrt{-d} \geq a>\sqrt{-d}$. The corresponding solutions of Painleve-IV equation must have the leading asymptotics $f(x) \sim-\lambda x / 3$ with subleading oscillations $\cos \left(\lambda x^{2} / \sqrt{3}+o(x)\right)$.

- Two normalizable zero modes of $\mathbf{a}^{-}$(and two sequences of levels) may exist for different solutions of Painleve-IV equation.

- Namely, for $\lambda>\sqrt{-d}$ the solution with asymptotics $f(x) \sim-\lambda x / 3$ provides the fall off of $\Psi_{-}^{(0)}(x), \Psi_{+}^{(0)}(x)$ if $a<\sqrt{-d}-2 \lambda$ and of $\Psi_{0}^{(0)}(x), \Psi_{+}^{(0)}(x)$ if $2 \lambda+\sqrt{-d}>a>2 \lambda-\sqrt{-d}$.

- For $\lambda>\sqrt{-d}$ the solution with asymptotics $f(x) \sim-\sqrt{-d} / 2 \lambda x$ generates two sequences of levels starting from $\Psi_{0}^{(0)}(x), \Psi_{-}^{(0)}(x)$ if $2 \lambda n+\sqrt{-d}<-a<$ $2 \lambda(n+1)-\sqrt{-d} ; n=0,1,2 \ldots$

- For $\lambda<\sqrt{-d}$ two sequences (from $\Psi_{0}^{(0)}(x)$ and $\Psi_{+}^{(0)}(x)$ ) are generated for two possible asymptotics $f(x) \sim \sqrt{-d} / 2 \lambda x$ and $f(x) \sim-\lambda x / 3$ if $2 \lambda+\sqrt{-d}>a>$ $\sqrt{-d}$.

- For $a \geq 2 \lambda+\sqrt{-d}$ and arbitrary positive $\lambda$ one sequence of equidistant levels will be realized with ground state $\Psi_{0}^{(0)}(x)$ and one of three asymptotics: $f(x) \sim-\lambda x / 3, \sim$ $\pm \sqrt{-d} / 2 \lambda x$.

- For $f(x) \sim-\lambda x / 3$ only one sequence of levels will be realized also for $\lambda=\sqrt{-d}, a= \pm \sqrt{-d}$ (starting from $\Psi_{0}^{(0)}(x)$ ), for $\lambda=\sqrt{-d}, a<\sqrt{-d}$ (starting from $\Psi_{-}^{(0)}(x)$ ) and for $\lambda<\sqrt{-d}, a \leq 2 \lambda+\sqrt{-d}$ (starting from $\left.\Psi_{+}^{(0)}(x)\right)$. 
- For asymptotic behavior $f(x) \sim-\sqrt{-d} / 2 \lambda x$ and $\lambda>\sqrt{-d}$ the spectrum consists of one sequence of levels if $|a+2 \lambda n|<\sqrt{-d}, n=0,1,2, \ldots$ (starts from $\left.\Psi_{-}^{(0)}(x)\right)$ or if $a=-\sqrt{-d}$ (starts from $\Psi_{0}^{(0)}$ ).

- Last, for $f(x) \sim \sqrt{-d} / 2 \lambda x$ one obtains one sequence with ground state $\Psi_{+}^{(0)}$ if $\lambda \leq \sqrt{-d}$ and $a<\sqrt{-d}$ or if $\lambda>\sqrt{-d}$ and $|a+2 \lambda n|<\sqrt{-d}, n=0,1,2, \ldots$

For specific values of parameter $a$ the normalizable zero mode of $\mathbf{a}^{+}$can cause truncation of one of two sequences ( with ground state $\Psi_{0}^{(0)}(x)$ ).

- Let us also remark that particular value $\alpha=-2 \lambda-\sqrt{-d}$, i.e. $E_{+}^{(0)}=0, E_{-}^{(0)}<0$, just corresponds to the case when Painleve-IV equation (155) has a class of particular solutions which coincide with solutions of the Riccati equation $g^{\prime}(y)=$ $g^{2}(y)+2 y g(y)+\sqrt{b}$. Substituting this Riccati equation into Eq.(152) one finds that potential is the pure harmonic oscillator: $V_{1}(x)=\lambda^{2} x^{2}-\lambda$.

- One additional singlet state satisfies the equation

$$
\mathbf{a}^{+} \Psi_{0}^{(0)}(x)=\mathbf{a}^{-} \Psi_{0}^{(0)}(x)=0 .
$$

For $\lambda>\sqrt{-d} \quad(\lambda<\sqrt{-d})$ it occurs when $a= \pm \sqrt{-d}$ and $\Psi_{0}^{(0)}(x)=\Psi_{2,1}(x)$ which entails the equation:

$$
f^{\prime}(x)=-2 f^{2}(x)-2 \lambda x f(x) \mp \sqrt{-d}
$$

with asymptotic behavior $f(x) \sim \mp \sqrt{-d} / 2 \lambda x$. The spacing between the two ground states $\Psi_{0}^{(0)}(x)$ and $\Psi_{-}^{(0)}(x)$ is: $\Delta E=2 \lambda \mp 2 \sqrt{-d}$.

- The doublet representation $\left(\Psi_{0}^{(0)}(x), \mathbf{a}^{+} \Psi_{0}^{(0)}(x)\right)$ of the spectrum generating algebra (135), (160) is built on solutions of the equation:

$$
\left(\mathbf{a}^{+}\right)^{2} \Psi_{0}^{(0)}(x)=\mathbf{a}^{-} \Psi_{0}^{(0)}(x)=0
$$

It may hold when $a=-2 \lambda-\sqrt{-d}$ for arbitrary positive value of $\lambda$ and when $a=-2 \lambda+\sqrt{-d}$ for $\lambda>\sqrt{-d}$. It is equivalent to $\mathbf{a}^{+} \Psi_{0}^{(0)}(x)=\Psi_{1,2}(x)$, which is satisfied when $f(x)$ obeys the following equation:

$$
\begin{aligned}
& 8 \lambda f^{2}(x)\left(f^{\prime}(x)+2 f^{2}(x)+2 \lambda x f(x)-2 \lambda \mp \sqrt{-d}\right)=\left(f^{\prime}(x)+2 f^{2}(x)+\right. \\
& 2 \lambda x f(x) \mp \sqrt{-d}) \cdot\left[\left(f^{\prime}(x)+2 f^{2}(x)+2 \lambda x f(x)-2 \lambda \mp \sqrt{-d}\right)\right. \\
& \left.\left(-f^{\prime}(x)+2 f^{2}(x)+2 \lambda x f(x)-2 \lambda \mp \sqrt{-d}\right)-4 \lambda(\lambda \pm \sqrt{-d})\right] .
\end{aligned}
$$

One can show that all solutions of this equation fulfill the Painleve-IV equation (154). These solutions have the asymptotics $f(x) \sim \pm \sqrt{-d} / 2 \lambda x$ and cannot have any (pole) singularity. The spectrum consists of a doublet $(0,2 \lambda)$ and infinite sequence $E_{n}= \pm 2 \sqrt{-d}+2(n+2) \lambda, n=0,1,2 \ldots$

Recently some higher-order generalizations of shape-invariance have been elaborated leading to the Painleve-V etc. equations [84], 85]. As well new solutions of Painleve-IV with complex parameters based on the third-order shape invariance have been found [86]. 


\section{Nonlinear SUSY for non-stationary Schrödinger equations}

\subsection{Linear SUSY and hidden symmetry}

In this Section our aim is to elucidate that many of the nonlinear SUSY constructions illustrated before can be implemented also in the Schrödinger time dependent framework [147], [87], [6], [148].

The first- and higher-order intertwining operators and the corresponding relations between non-stationary one-dimensional Schrödinger operators can be introduced straightforwardly. But, as compared to the stationary case, already the first-order intertwining relations imply some hidden symmetry $\$$ which leads to a specific quantum dynamics when the evolution is described by quantum orbits and results in the $R$ separation of variables [87]. In turn second-order intertwining operators [87],[149] and the corresponding non-linear SUSY give rise to the quantum motion governed by the spectrum generating algebras. We start with the non-stationary Schrödinger operator

$$
\mathcal{S}[V]=i \partial_{t}+\partial_{x}^{2}-V(x, t)
$$

Here $\partial_{t}=\partial / \partial t$ and $\partial_{x}=\partial / \partial x$ denote the partial derivatives with respect to time and position. When they are applied to some function $f$, the following notations for these derivatives are used: $\dot{f}(x, t)=\left(\partial_{t} f\right)(x, t)$ and $f^{\prime}(x, t)=\left(\partial_{x} f\right)(x, t)$.

The intertwining operator of first order [87] is given by

$$
q_{t}^{+}=\xi_{0}(x, t) \partial_{t}+\xi_{1}(x, t) \partial_{x}+\xi_{2}(x, t)
$$

with, in general, complex-valued functions $\xi_{0}, \xi_{1}$ and $\xi_{2}$. The possibility of a complexification of the intertwining (Darboux) (first and also higher order) operator was emphasized by [149].

For the Schrödinger operator (163) the intertwining relation reads

$$
\mathcal{S}\left[V^{(0)}\right] q_{t}^{+}=q_{t}^{+} \mathcal{S}\left[V^{(1)}\right],
$$

where the functions $\xi_{i}(i=0,1,2)$ and $V^{(0),(1)}$ are not independent of each other. It can be also represented in the SUSY form Eqs. (11),(12) when the stationary Hamiltonians $h^{(0),(1)}$ are extended to the Schrödinger operators $\mathcal{S}\left[V^{(0),(1)}\right]$, then

$$
\left[\hat{\mathcal{S}}_{t}, Q_{t}^{-}\right]=0, \quad \hat{\mathcal{S}}_{t}=\left(\begin{array}{cc}
\mathcal{S}\left[V^{(0)}\right] & 0 \\
0 & \mathcal{S}\left[V^{(1)}\right]
\end{array}\right), \quad Q_{t}^{-}=\left(\begin{array}{cc}
0 & q_{t}^{+} \\
0 & 0
\end{array}\right) .
$$

After inserting (163) and the intertwining operator (164) into relation (165) it can be derived that $\xi_{0}$ and $\xi_{1}$ may depend only on time, i.e. $\xi_{0}^{\prime}=0=\xi_{1}^{\prime}$. Then the assumption that $\xi_{0}$ does not vanish identically would entail that the potential difference $V^{(0)}-V^{(1)}$ does depend on time only. This is a rather trivial case and therefore let

$\S$ Symmetry properties of the time dependent Schrödinger equation were studied in general in [150], 151] (and references therein). 
us take $\xi_{0} \equiv 0$. Making the appropriate choice of variables $\xi_{1}(t)=e^{i \beta(t)} \rho(t)$ and $\xi_{2}(x, t)=e^{i \beta(t)} \rho(t) \omega^{\prime}(x, t)$ with real $\beta$, positive $\rho$ and complex $\omega$ functions one finds

$$
\begin{aligned}
& V^{(0)}(x, t)=\omega^{2}(x, t)+\omega^{\prime \prime}(x, t)-i \dot{\omega}(x, t)+\alpha(t)-\dot{\beta}(t)+i \dot{\rho}(t) / \rho(t), \\
& V^{(1)}(x, t)=\omega^{\prime 2}(x, t)-\omega^{\prime \prime}(x, t)-i \dot{\omega}(x, t)+\alpha(t)
\end{aligned}
$$

where $\alpha$ is a time-dependent complex-valued integration constant. One may set 87 ] $\beta \equiv 0$ without loss of generality. Furthermore, one may also remove $\alpha \rightarrow 0$ as being absorbed in $\omega$ by the shift $\omega \rightarrow \omega-i \int d t \alpha$. Finally, we are left with

$$
\begin{aligned}
& V^{(0)}(x, t)=\omega^{2}(x, t)+\omega^{\prime \prime}(x, t)-i \dot{\omega}(x, t)+i \dot{\rho}(t) / \rho(t), \\
& V^{(1)}(x, t)=\omega^{\prime 2}(x, t)-\omega^{\prime \prime}(x, t)-i \dot{\omega}(x, t) .
\end{aligned}
$$

Here the super-potential $\omega$ is not arbitrary as the potentials are assumed to be real. It could be achieved by taking a stationary real super-potential. However this choice leads to the stationary SUSY QM discussed previously. Therefore, we consider a complex super-potential

$$
\omega(x, t)=\omega_{R}(x, t)+i \omega_{I}(x, t)
$$

with real functions $\omega_{R}$ and $\omega_{I}$. The reality condition $\operatorname{Im} V^{(0)}=\operatorname{Im} V^{(1)}=0$ is fulfilled if

$$
2\left(\omega_{R}\right)^{\prime \prime}+\dot{\rho} / \rho=0, \quad 2\left(\omega_{R}\right)^{\prime}\left(\omega_{I}\right)^{\prime}-\left(\omega_{I}\right)^{\prime \prime}-\omega_{R}=0,
$$

which can be integrated to

$$
\begin{aligned}
\omega_{I}(x, t) & =-\frac{1}{4} \frac{\dot{\rho}(t)}{\rho(t)} x^{2}+\frac{1}{2} \rho(t) \dot{\mu}(t) x+\gamma(t), \\
\omega_{R}(x, t) & =\frac{1}{2} \ln \rho(t)+K(x / \rho(t)+\mu(t)),
\end{aligned}
$$

where $\mu$ and $\gamma$ are arbitrary real functions of time and $K$ is an arbitrary real function of the variable $y=x / \rho+\mu$. In terms of these functions the final form of the two partner potentials is

$$
\begin{aligned}
V^{(0),(1)}(x, t)= & \frac{1}{\rho^{2}(t)}\left[K^{\prime 2}(y) \pm K^{\prime \prime}(y)\right] \\
& -\frac{\ddot{\rho}(t)}{4 \rho(t)} x^{2}+\left(\dot{\rho}(t) \dot{\mu}(t)+\frac{\rho(t) \ddot{\mu}(t)}{2}\right) x-\frac{\rho^{2}(t) \dot{\mu}^{2}(t)}{4}+\dot{\gamma}(t)
\end{aligned}
$$

and the intertwining operator reads

$$
q_{t}^{+}(x, t)=\rho(t) \partial_{x}+K^{\prime}(x / \rho(t)+\mu(t))-\frac{i}{2}\left(\dot{\rho}(t) x-\rho^{2}(t) \dot{\mu}(t)\right) .
$$

Let us demonstrate that the non-stationary Schrödinger equation $\mathcal{S}\left[V^{(0),(1)}\right] \psi^{(0),(1)}=0$ with potentials given in Eq. (172) (which is equivalent to the intertwining (165)) admits a separation of variables. Indeed, after the transformation

$y=x / \rho(t)+\mu(t), \quad \psi^{(0),(1)}(x, t)=\frac{1}{\sqrt{\rho(t)}} e^{-i \omega_{I}(x, t)} \phi^{(0),(1)}(y, t) \equiv \Omega(x, t) \phi^{(0),(1)}(y, t)(174)$ 
this Schrödinger equation becomes quasi-stationary [152]

$$
i \rho^{2}(t) \partial_{t} \phi^{(0),(1)}(y, t)=\left[-\partial_{y}^{2}+K^{\prime 2}(y) \pm K^{\prime \prime}(y)\right] \phi^{(0),(1)}(y, t),
$$

which is obviously separable in $y$ and $t$. Hence, the solutions of the original Schrödinger equations have the general form $\psi(x, t)=\Omega(y, t) Y(y) T(t)$ which is known as the $R$-separation of variables [153]. In other words, for any pair of Schrödinger operators $\mathcal{S}\left[V^{(0),(1)}\right]$, which admits a first-order intertwining relation (165) there exists a transformation (174) to some new coordinate in which the potentials become stationary (see also [148]).

This $R$-separation of variables is certainly related to the existence of a symmetry operator for the super-Hamiltonian. First, one can directly verify the adjoint intertwining relation for real potentials,

$$
q_{t}^{-} \mathcal{S}\left[V^{(0)}\right]=\mathcal{S}\left[V^{(1)}\right] q_{t}^{-}
$$

where

$q_{t}^{-} \equiv\left(q_{t}^{+}\right)^{\dagger}=-\rho(t) \partial_{x}+K^{\prime}(x / \rho(t)+\mu(t))+\frac{i}{2}\left(\dot{\rho}(t) x-\rho^{2}(t) \dot{\mu}(t)\right)$.

Then from (165), (166) and (176) we obtain the closure of the SUSY algebra,

$$
\left\{Q_{t}, \bar{Q}_{t}\right\}=\mathcal{R}_{t}, \quad\left[\hat{\mathcal{S}}_{t}, \mathcal{R}_{t}\right]=0, \quad \bar{Q}_{t}=\left(Q_{t}\right)^{\dagger},
$$

where the symmetry operator $\mathcal{R}_{t}$ has the following components

$$
\begin{aligned}
& R_{t}^{ \pm}=q_{t}^{ \pm} q_{t}^{\mp}=-\rho^{2}(t) \partial_{x}^{2}+\frac{i}{2}\left(\rho \dot{\rho}(t)\left\{x, \partial_{x}\right\}-2 \rho^{3}(t) \dot{\mu}(t) \partial_{x}\right) \\
& +\left[K^{\prime}(x / \rho(t)+\mu(t))\right]^{2} \pm K^{\prime \prime}(x / \rho(t)+\mu(t))+\frac{1}{4}\left(\dot{\rho}(t) x-\rho^{2}(t) \dot{\mu}(t)\right)^{2} \\
= & \exp \left\{-i \omega_{I}(x, t)\right\}\left[-\partial_{y}^{2}+K^{\prime 2}(y) \pm K^{\prime \prime}(y)\right] \exp \left\{i \omega_{I}(x, t)\right\} .
\end{aligned}
$$

Thus the quasi-stationary Hamiltonians in Eq. (175) are just unitary equivalent to the symmetry operators $R_{t}^{ \pm}$. It means that the supersymmetry entails the separation of variables because it provides a new symmetry for the super-Hamiltonian. As consequence the quantum dynamics splits in orbits with a given eigenvalue of the symmetry operator.

\subsection{Second-order intertwining for stationary potentials: spectrum generating algebra}

Let us relate a pair of Schrödinger operators $\mathcal{S}\left[V^{(0)}\right]$ and $\mathcal{S}\left[V^{(1)}\right]$ by second-order (intertwining) operators of the form,

$$
q_{t}^{+}(x, t)=G(x, t) \partial_{x}^{2}-2 F(x, t) \partial_{x}+B(x, t) .
$$

We will explore the existence of the time-dependent SUSY charges with appearance of the spectrum generating (oscillator like) algebras for corresponding Hamiltonians.

As in the first-order case it can be shown [87] that the inclusion of an additional term of first order in $\partial_{t}$ leads to the trivial solutions when the difference $V^{(0)}-V^{(1)}$ depends on the time $t$ only. Furthermore, from the intertwining relation (165) with 
the above $q_{t}^{+}$one can conclude that the function $G$ cannot depend on $x$ and it is even possible to exclude a phase. In other words, without loss of generality $G(x, t) \equiv g(t)$ and accordingly, from now on, an intertwining operator can be reduced to,

$$
q_{t}^{+}(x, t)=g(t) \partial_{x}^{2}-2 F(x, t) \partial_{x}+B(x, t) .
$$

In [87] particular solutions of the intertwining relation (165) were constructed with $q_{t}^{+}$ as given above. In this section we shall analyze the solutions of the intertwining relation (165) for the case where both potentials $V^{(0)}$ and $V^{(1)}$ are stationary, i.e. do not depend on $t$.

One class of such solutions is known from [47]. Assuming a supercharge $q_{t}^{+}$with real coefficient functions independent on $t$, one finds that the corresponding solutions of (165) $)$ coincide with those of the stationary intertwining relations $\left(-\partial_{x}^{2}+V^{(0)}(x)\right) q^{+}(x)=$ $q^{+}(x)\left(-\partial_{x}^{2}+V^{(1)}(x)\right)$.

Here we are interested in more general solutions of (165) when operators $q_{t}^{+}$depend on $t$,

$$
\left(i \partial_{t}-h^{(0)}\right) q_{t}^{+}(x, t)=q_{t}^{+}(x, t)\left(i \partial_{t}-h^{(1)}\right)
$$

with stationary Hamiltonians $h^{(0),(1)}=-\partial_{x}^{2}+V^{(0),(1)}(x)$ but time-dependent intertwining operators.

Let us employ the suitable ansatz with simple $t$-dependence in (181),

$$
q_{t}^{+}(x, t)=k^{+}(x)+A(t) p^{+}(x),
$$

where,

$$
k^{+}(x) \equiv \partial_{x}^{2}-2 f(x) \partial_{x}+\tilde{b}(x)-f^{\prime}(x), \quad p^{+}(x) \equiv-\partial_{x}+\chi(x) .
$$

Here all functions besides $A$ are considered to be real. We also assume $A \not \equiv 0$. With this ansatz the intertwining relation (182) can be shown [87] to yield,

$$
\begin{aligned}
& i \dot{A}=2 \tilde{m}+2 m A, \\
& k^{+} h^{(0)}-h^{(1)} k^{+}-=2 \tilde{m} p^{+}, \\
& h^{(0)} p^{+}-p^{+} h^{(1)}=2 m p^{+},
\end{aligned}
$$

with real constants $\tilde{m}$ and $m$.

We find it interesting to focus on the case $m \neq 0$ to explore certain spectrum generating algebras. The first equation in (185) immediately leads to

$$
A(t)=m_{0} e^{-2 i m t}-\tilde{m} / m
$$

with a real $m_{0}$ and,

$q_{t}^{+}(x, t)=\partial_{x}^{2}-\left(2 f(x)+\frac{\tilde{m}}{m}\right) \partial_{x}+\tilde{b}(x)-f^{\prime}(x)+\frac{\tilde{m}}{m} \chi(x)-m_{0} e^{-2 i m t} p^{+}(x)$.

Without loss of generality we may set $\tilde{m}=0$ because a non-vanishing $\tilde{m}$ may always be absorbed via a proper redefinition of $f$ and $\tilde{b}$, i.e. of the operator $k^{+}$.

As a consequence, the second relation in (185) leads to a second-order intertwining between $h^{(0)}$ and $h^{(1)}$. This has already been considered in [47] and it was found that 
the potentials $V^{(0)}, V^{(1)}$ and the function $\tilde{b}$ can be expressed in terms of $f$ and two arbitrary real constants $a$ and $d$ as in Subsect.5.1, Eq.(105),

$$
\begin{aligned}
& V^{(0),(1)}(x)=\mp 2 f^{\prime}(x)+f^{2}(x)+\frac{f^{\prime \prime}(x)}{2 f(x)}-\frac{f^{\prime 2}(x)}{4 f^{2}(x)}-\frac{d}{4 f^{2}(x)}-a, \\
& \tilde{b}(x)=f^{2}(x)-\frac{f^{\prime \prime}(x)}{2 f(x)}+\frac{f^{\prime 2}(x)}{4 f^{2}(x)}+\frac{d}{4 f^{2}(x)} .
\end{aligned}
$$

The corresponding second-order SUSY algebra generated by the supercharge $K$ is similar to (107).

One may find some similarities between the present intertwining algebra (185) and the extended SUSY relations discussed in Section 5. But we emphasize that now for $m \neq 0$ the last relation in (185) does not generate a second SUSY. Rather it creates the equivalence of relatively shifted spectra of two Hamiltonians $h^{(0)}$ and $h^{(1)}$ which is typical for spectrum generating algebras emerging for the shape-invariant Hamiltonians (Sect.6). Specifically,

$$
p^{+} p^{-}=h^{(0)}-m+c ; \quad p^{-} p^{+}=h^{(1)}+m+c,
$$

where $c$ is a real constant. Therefore the reflectionless potentials found in Section 5 are produced only in the limit of $m=0$.

The genuine spectrum generating algebra for stationary Hamiltonians $h^{(0),(1)}$ can be derived from Eq. (185)

$$
\begin{aligned}
& {\left[h^{(0)}, \mathbf{a}_{(0)}^{-}\right]=-2 m \mathbf{a}_{(0)}^{-}, \quad \mathbf{a}_{(0)}^{-} \equiv k^{+} p^{-},} \\
& {\left[h^{(0)}, \mathbf{a}_{(0)}^{+}\right]=2 m \mathbf{a}_{(0)}^{+}, \quad \mathbf{a}_{(0)}^{+} \equiv p^{+} k^{-},} \\
& {\left[h^{(1)}, \mathbf{a}_{(1)}^{-}\right]=2 m \mathbf{a}_{(1)}^{-}, \quad \mathbf{a}_{(1)}^{-} \equiv k^{-} p^{+},} \\
& {\left[h^{(1)}, \mathbf{a}_{(1)}^{+}\right]=-2 m \mathbf{a}_{(1)}^{+}, \quad \mathbf{a}_{(1)}^{+} \equiv p^{-} k^{+},}
\end{aligned}
$$

where $p^{-}=\left(p^{+}\right)^{\dagger}, k^{-}=\left(k^{+}\right)^{\dagger}$ and $\mathbf{a}_{(0,1)}^{-}=\left(\mathbf{a}_{(0,1)}^{+}\right)^{\dagger}$.

The closure of this spectrum generating algebra is a polynomial deformation of Heisenberg algebra [83] (see Subsect. 6.4),

$$
\left[\mathbf{a}_{(0,1)}^{+}, \mathbf{a}_{(0,1)}^{-}\right]=P^{(0,1)}\left(h^{(0,1)}\right) \text {. }
$$

The explicit form of the polynomials $P^{(0,1}$ can be obtained with the help of Eqs. (185) and (189) for $\tilde{m}=0$. For instance,

$$
\begin{aligned}
& \mathbf{a}_{(0)}^{+} \mathbf{a}_{(0)}^{-}=\left(h^{(0)}-m+c\right)\left[\left(h^{(0)}-2 m+a\right)^{2}+d\right] ; \\
& \mathbf{a}_{(0)}^{-} \mathbf{a}_{(0)}^{+}=\left(h^{(0)}+m+c\right)\left[\left(h^{(0)}+a\right)^{2}+d\right],
\end{aligned}
$$

where the notations from Eqs. (107) and (189) are employed. The polynomials $P^{(0,1)}$ turn out to be different for the isospectral partners $h^{ \pm}$,

$P^{(0)}\left(h^{(0)}\right)=-6 m\left(h^{(0)}\right)^{2}+4 m(2 m-2 a-c) h^{(0)}-2 m\left[a^{2}+d+2(a-m)(c-m)\right] ;$

$P^{(1)}\left(h^{(1)}\right)=-6 m\left(h^{(1)}\right)^{2}-4 m(2 m+2 a+c) h^{(1)}-2 m\left[a^{2}+d+2(a+m)(c+m)\right]$.

Hence the two spectrum generating algebras are, in general, different that is essentially due to the shift in intertwining relations (189). When comparing to the equations in 
Subsection 6.4 we find that the generating algebras (192), (193) are in fact originating from the third-order shape invariance.

There is a formal discrete symmetry between their constants and Hamiltonians $h^{(0)}, a, c \Longrightarrow-h^{(1)},-a,-c$.

The intertwining relation (182) and its adjoint give rise to the symmetry operators $q_{t}^{+} q_{t}^{-}$and $q_{t}^{-} q_{t}^{+}$for $\left(i \partial_{t}-h^{(0)}\right)$ and $\left(i \partial_{t}-h^{(1)}\right)$, respectively. Using Eqs. (107), (186), (189) and after elimination of polynomials of the Hamiltonians $h^{(0,1)}$ these symmetry operators may be reduced to,

$$
\begin{aligned}
& R^{(0)}(x, t)=m_{0}\left[e^{2 i m t} \mathbf{a}_{(0)}^{-}+e^{-2 i m t} \mathbf{a}_{(0)}^{+}\right], \\
& R^{(1)}(x, t)=m_{0}\left[e^{2 i m t} \mathbf{a}_{(1)}^{-}+e^{-2 i m t} \mathbf{a}_{(1)}^{+}\right] .
\end{aligned}
$$

As our potentials do not depend on time the time derivatives $\dot{R}^{(0,1)}(x, t)$ of Hermitian symmetry operators $R^{(0,1)}(x, t)$ form the independent set of hermitian symmetry operators which do not commute between themselves. Similar results have also been obtained in [151] using a different approach. We see that the non-stationary SUSY delivers the time-dependent hidden symmetry operators [14],

$$
e^{2 i m t} \mathbf{a}_{(0,1)}^{-}=\frac{1}{2 m_{0}} R^{(0,1)}(x, t)-\frac{i}{4 m m_{0}} \dot{R}^{(0,1)}(x, t),
$$

which give the entire set of spectrum generating algebras previously found for the thirdorder shape invariance. Thus hidden symmetries for nonstationary Schrödinger superHamiltonians give the universal framework for both stationary hidden symmetries and for spectrum generating algebras of shape invariant SUSY systems.

\section{Polynomial SUSY QM in $d=2$}

\subsection{Supercharges of second order in derivatives: generalities}

It was shown in Section 2, that multidimensional generalization of SUSY QM includes both scalar and matrix Hamiltonians, and some physical examples of matrix problems, incorporated into SUSY QM, were given in Subsections 2.2 and 2.5. In general, the construction does not provide any intertwining and therefore any direct relations between spectra and wave functions of two scalar components of super-Hamiltonian, if we are not interested in models with separation of variables. But such opportunity can be opened by applying and development of the ideas of polynomial SUSY of Section 3 for higher dimensionality of space. In particular, this approach turned out [88 - 92, [94], [95], 97] - [100], [102] - [105] to be very fruitful for study of nontrivial spectral problems in the case of $d=2$ (see also [13], [16]).

Thus, in order to get rid of matrix component of two-dimensional superHamiltonian, we shall explore the supercharges of second order in partial derivatives. The simplest variant of second order supercharges - of reducible form $q^{ \pm}=\left(q_{i}^{ \pm}\right)\left(\tilde{q}_{i}^{ \pm}\right)$is not very promising: the intertwined partner Hamiltonians differ by a trivial constant only, and both of them admit the separation of variables (see details in [88, [89]). By 
this reason, here we are interested in general irreducible second order components of supercharges,

$$
q^{+}=g_{i k}(\vec{x}) \partial_{i} \partial_{k}+C_{i}(\vec{x}) \partial_{i}+B(\vec{x}) ; \quad q^{-} \equiv\left(q^{+}\right)^{\dagger},
$$

with real "metrics" $g_{i k}(\vec{x})$ and coefficient functions $C_{i}(\vec{x}), B(\vec{x})$. The familiar intertwining relations

$$
h^{(0)} q^{+}=q^{+} h^{(1)} ; \quad q^{-} h^{(0)}=h^{(1)} q^{-} .
$$

for two scalar two-dimensional Hamiltonians of Schrödinger form,

$$
h^{(0),(1)}=-\Delta^{(2)}+V^{(0),(1)}(\vec{x})
$$

can be rewritten as a system of nonlinear partial differential equations for $g_{i k}(\vec{x})$, the coefficient functions $C_{i}(\vec{x}), B(\vec{x})$ and potentials $V^{(0)(1)}(\vec{x})$. The general solution for the "metrics" $g_{i k}(\vec{x})$ is [88, [89,

$g_{11}=a x_{2}^{2}+a_{1} x_{2}+b_{1} ; g_{22}=a x_{1}^{2}+a_{2} x_{1}+b_{2} ; g_{12}=-\frac{1}{2}\left(2 a x_{1} x_{2}+a_{1} x_{1}+a_{2} x_{2}\right)+b_{3}$,

where $\left(a, a_{i}, b_{i}=\right.$ Const $)$. Thus, the senior in derivative part of supercharges belongs to the $E(2)$ - universal enveloping algebra [153]. We distinguish four different (inequivalent) classes in second derivatives [88], [89],

$$
\begin{aligned}
& q^{(1)+}=\gamma\left(P_{1}^{2}+P_{2}^{2}\right)+C_{i} \partial_{i}+B ; \\
& q^{(2)+}=\alpha P_{1}^{2}+(\alpha+\gamma) P_{2}^{2}+C_{i} \partial_{i}+B ; \\
& q^{(3)+}=\gamma\left\{J_{3}, P_{1}\right\}+\alpha\left(P_{1}^{2}+P_{2}^{2}\right)+C_{i} \partial_{i}+B ; \\
& q^{(4)+}=\gamma J_{3}^{2}+\beta P_{1}^{2}+\alpha P_{2}^{2}+C_{i} \partial_{i}+B,
\end{aligned}
$$

where $J_{3}$ and $P_{1}, P_{2}$ are generators of rotations and translations, correspondingly, and $\gamma \neq 0$.

The solutions (199) must be inserted into six other equations which followed from the intertwining relations (197),

$$
\begin{aligned}
& \partial_{i} C_{k}(\vec{x})+\partial_{k} C_{i}(\vec{x})+\Delta^{(2)} g_{i k}(\vec{x})-\left(V^{(0)}(\vec{x})-V^{(1)}(\vec{x})\right) g_{i k}(\vec{x})=0 ; \\
& \Delta^{(2)} C_{i}(\vec{x})+2 \partial_{i} B(\vec{x})+2 g_{i k}(\vec{x}) \partial_{k} V^{(1)}(\vec{x})-\left(V^{(0)}(\vec{x})-V^{(1)}(\vec{x})\right) C_{i}(\vec{x})=0 ; \\
& \Delta^{(2)} B(\vec{x})+g_{i k}(\vec{x}) \partial_{k} \partial_{i} V^{(1)}(\vec{x})+C_{i}(\vec{x}) \partial_{i} V^{(1)}(\vec{x})-\left(V^{(0)}(\vec{x})-V^{(1)}(\vec{x})\right) B(\vec{x})=0 .
\end{aligned}
$$

It is clear that we have no chances to solve these equations with general form (199) of $g_{i k}(\vec{x})$. Therefore, one is forced to consider the simplest cases of constant metrics $g_{i k}$ looking for particular solutions for functions $C_{i}(\vec{x}), B(\vec{x}), V^{(0)}(\vec{x}), V^{(1)}(\vec{x})$.

The first sample of constant metrics is the elliptic (Laplace) one corresponding to (200), $g_{i k}=\delta_{i k}$. In this case, the system (204) - (206) is essentially simplified, so that all coefficient functions can be found analytically [88], 89]. In particular, the combination $C \equiv C_{1}+i C_{2}$ depends only on $z=x_{1}+i x_{2}$, and it has the specific form,

$$
C^{2}(z)=a z^{2}+b z+c
$$

where $a$ is real and $b, c$ complex constants. All other functions can be also found explicitly, but depending on values of constants in (207), both potentials allow 
$R$-separation [153] of variables - in elliptic (for $b=0, a \neq 0$ ), parabolic (for $a=0, b \neq 0)$ or polar $(b=c=0, a \neq 0)$ coordinates. The situation with both $a \neq 0, b \neq 0$ is reduced to the first variant by a suitable constant shift of coordinates. The case of polar coordinates just corresponds to reducible supercharges $q_{i}^{ \pm} \tilde{q}_{i}^{ \pm}$. Thus, for all possible values of coefficients the class of two-dimensional problems with elliptic metrics of supercharges can be reduced to two one-dimensional models, and it will not be considered below.

\subsection{Hyperbolic (Lorentz) metrics}

8.2.1. Supercharges with twisted reducibility. The constant metrics of hyperbolic (Lorentz) type, $g_{i k}=\operatorname{diag}(+1,-1)$ gives much more interesting models [89], [91], [90]. One specific class of such solutions of intertwining relations (197) with the so called supercharges with twisted reducibility.

$$
q^{-}=\left(q^{+}\right)^{\dagger}=q_{l}^{+}\left(\sigma_{3}\right)_{l k} \tilde{q}_{k}^{-}=\left(-\partial_{l}+\partial_{l} W(\vec{x})\right)\left(\sigma_{3}\right)_{l k}\left(+\partial_{k}+\partial_{k} \widetilde{W}(\vec{x})\right),
$$

was studied in papers [94], 97], [99]. In (208), $W(\vec{x}), \widetilde{W}(\vec{x})$ are two different functions (superpotentials), $\sigma_{3}$ is the Pauli matrix, and summation over $k, l=1,2$ is implied. This form of supercharges can be considered as a generalization of the simplest reducibility for elliptic metrics, mentioned in the beginning of previous Subsection. In contrast to that case, the form (208) leads to very nontrivial models, which do not allow the standard separation of variables. This construction of supercharges can be interpreted as obtained after gluing of two systems of two-dimensional SUSY QM of Subsection 2.1 with first order supercharges with different superpotentials $W, \widetilde{W}$, correspondingly. These systems are glued by their matrix components of super-Hamiltonian: these components are taken coinciding but up to an unitary constant matrix rotation by $\sigma_{3}$. In such a case, both Hamiltonians $h^{(0)}=\tilde{q}_{l}^{+} \tilde{q}_{l}^{-}$and $h^{(1)}=q_{l}^{+} q_{l}^{-}$are quasifactorized in terms of $\tilde{q}_{i}^{ \pm}$and $q_{i}^{ \pm}$, correspondingly.

It was shown in [94], 97] in a general form that Eqs.(197) and (208) lead to the following representation for superpotentials $W, \tilde{W}$ in terms of four functions $\mu_{1,2, \pm}$,

$$
\begin{aligned}
& \chi=\mu_{1}\left(x_{1}\right)+\mu_{2}\left(x_{2}\right)+\mu_{+}\left(x_{+}\right)+\mu_{-}\left(x_{-}\right), \\
& \tilde{\chi}=\mu_{1}\left(x_{1}\right)+\mu_{2}\left(x_{2}\right)-\mu_{+}\left(x_{+}\right)-\mu_{-}\left(x_{-}\right),
\end{aligned}
$$

where the light cone variables $x_{ \pm}=x_{1} \pm x_{2}$ were introduced. These functions $\mu_{1,2, \pm}$ have to satisfy the following equation,

$$
\mu_{1}^{\prime}\left(x_{1}\right)\left[\mu_{+}^{\prime}\left(x_{+}\right)+\mu_{-}^{\prime}\left(x_{-}\right)\right]+\mu_{2}^{\prime}\left(x_{2}\right)\left[\mu_{+}^{\prime}\left(x_{+}\right)-\mu_{-}^{\prime}\left(x_{-}\right)\right]=0,
$$

where $\mu^{\prime}(x)$ means derivative over the argument. By substitutions $\phi \equiv \mu^{\prime}$, it is evident that we deal with a purely functional equation with no derivatives,

$$
\phi_{1}\left(x_{1}\right)\left[\phi_{+}\left(x_{+}\right)+\phi_{-}\left(x_{-}\right)\right]=-\phi_{2}\left(x_{2}\right)\left[\phi_{+}\left(x_{+}\right)-\phi_{-}\left(x_{-}\right)\right] .
$$


Each solution of Eq.(210) gives the corresponding solution of the intertwining relations (197) for the potentials $V, \widetilde{V}$ and for the supercharges $q^{ \pm}$,

$$
\begin{aligned}
V^{(0)}(\vec{x})= & \left(\phi_{1}^{2}\left(x_{1}\right)-\phi_{1}^{\prime}\left(x_{1}\right)\right)+\left(\phi_{2}^{2}\left(x_{2}\right)-\phi_{2}^{\prime}\left(x_{2}\right)\right)+\left(\phi_{+}^{2}\left(x_{+}\right)+\sqrt{2} \phi_{+}^{\prime}\left(x_{+}\right)\right)+ \\
& +\left(\phi_{-}^{2}\left(x_{-}\right)+\sqrt{2} \phi_{-}^{\prime}\left(x_{-}\right)\right), \\
V^{(1)}(\vec{x})= & \left(\phi_{1}^{2}\left(x_{1}\right)-\phi_{1}^{\prime}\left(x_{1}\right)\right)+\left(\phi_{2}^{2}\left(x_{2}\right)-\phi_{2}^{\prime}\left(x_{2}\right)\right)+\left(\phi_{+}^{2}\left(x_{+}\right)-\sqrt{2} \phi_{+}^{\prime}\left(x_{+}\right)\right)+ \\
& +\left(\phi_{-}^{2}\left(x_{-}\right)-\sqrt{2} \phi_{-}^{\prime}\left(x_{-}\right)\right), \\
Q^{ \pm}=\partial_{1}^{2}- & \partial_{2}^{2} \pm \sqrt{2}\left(\phi_{+}\left(x_{+}\right)+\phi_{-}\left(x_{-}\right)\right) \partial_{1} \mp \sqrt{2}\left(\phi_{+}\left(x_{+}\right)-\phi_{-}\left(x_{-}\right)\right) \partial_{2}- \\
- & \left(\phi_{1}^{2}\left(x_{1}\right)-\phi_{1}^{\prime}\left(x_{1}\right)\right)+\left(\phi_{2}^{2}\left(x_{2}\right)-\phi_{2}^{\prime}\left(x_{2}\right)\right)+2 \phi_{+}\left(x_{+}\right) \phi_{-}\left(x_{-}\right) .
\end{aligned}
$$

Thus, in order to find the systems with intertwining (197) by supercharges of the form (208), it is necessary to solve (210). This equation seems to be rather complicated, but it appeared to be solvable in a general form (see details in [99]). In particular, it was shown that $\phi_{1,2}(x)$ are defined from solutions of the first order nonlinear differential equation,

$$
\left(\phi^{\prime}\right)_{1,2}^{2}=a \phi_{1,2}^{4}+b \phi_{1,2}^{2}+c,
$$

where $a, b, c$ are arbitrary real constants. All solutions of this equation can be expressed in terms of elliptic functions, and they are described for different ranges of parameters, for example, in Appendix B of [154]. In turn, the functions $\phi_{ \pm}$are defined uniquely by solution of (213): they also satisfy equation of the form (213), but with different constant coefficients. Depending on the values of $a, b, c$, solutions $\phi$ can be functions of their argument with finite or infinite period. Corresponding models were studied in detail in [99] (periodic potentials) and in 994, 97] (potentials with infinite period).

8.2.2. Irreducible supercharges. Let us consider now the most general supercharges with hyperbolic (Lorentz) metrics without any kind of reducibility [88, [89], [91], [13]. The light cone variables $x_{ \pm} \equiv x_{1} \pm x_{2}$ are again the most convenient to look for the solution of intertwining relations. In particular, it follows from the system (204) - (206) that the combinations $C_{ \pm} \equiv C_{1} \mp C_{2}$ depend only on one argument:

$$
C_{+}=C_{+}\left(x_{+}\right), \quad C_{-}=C_{-}\left(x_{-}\right) .
$$

Then, the whole system (204) - (206) can be rewritten in a very compact form of two equations for functions $C_{ \pm}\left(x_{ \pm}\right)$and an auxiliary function $F(\vec{x})$,

$$
\begin{aligned}
& \partial_{-}\left(C_{-} F\right)=-\partial_{+}\left(C_{+} F\right) ; \\
& \partial_{+}^{2} F=\partial_{-}^{2} F .
\end{aligned}
$$

It is easy to write the general solution $F(\vec{x})$ of Eq.(216): it is expressed in terms of two arbitrary functions,

$$
F=F_{1}\left(x_{+}+x_{-}\right)+F_{2}\left(x_{+}-x_{-}\right),
$$


both are defined up to an arbitrary real constant, $F_{1} \rightarrow F_{1}+\gamma, F_{2} \rightarrow F_{2}-\gamma$. The potentials $V^{(1),(2)}(\vec{x})$ and the function $B(\vec{x})$ are expressed in terms of four functions $F_{1}\left(2 x_{1}\right), F_{2}\left(2 x_{2}\right)$ and $C_{ \pm}\left(x_{ \pm}\right)$, which must satisfy the only equation (215),

$V^{(0),(1)}= \pm \frac{1}{2}\left(C_{+}^{\prime}+C_{-}^{\prime}\right)+\frac{1}{8}\left(C_{+}^{2}+C_{-}^{2}\right)+\frac{1}{4}\left(F_{2}\left(x_{+}-x_{-}\right)-F_{1}\left(x_{+}+x_{-}\right)\right) ;$

$B=\frac{1}{4}\left(C_{+} C_{-}+F_{1}\left(x_{+}+x_{-}\right)+F_{2}\left(x_{+}-x_{-}\right)\right)$,

where $C^{\prime}$ means derivative in its argument. Although Eq.(215) has a compact form, it is very nontrivial being the functional-differential equation: the functions each depend on specific argument. As a rule, equations of this kind have no regular recipe for solution, and this is the case for Eq.(215). Usually, one may try to solve such equation starting from different suitable anzatses for functions $C_{ \pm}\left(x_{ \pm}\right), F(\vec{x})$.

1) Let us choose $C_{-}=0$, then from (215) one obtains $F=\phi\left(x_{-}\right) / C_{+}\left(x_{+}\right)$. After inserting into Eq.(216) the separation of variables is possible, and particular solution reads,

$$
\begin{aligned}
& C_{+}\left(x_{+}\right)=\frac{1}{\delta_{1} \exp \left(\sqrt{\lambda} \cdot x_{+}\right)+\delta_{2} \exp \left(-\sqrt{\lambda} \cdot x_{+}\right)} \\
& F_{1,2}(2 x)=\delta_{1} \sigma_{1,2} \exp (2 \sqrt{\lambda} x)+\delta_{2} \sigma_{2,1} \exp (-2 \sqrt{\lambda} x),
\end{aligned}
$$

where the Greek letters - arbitrary constants: depending on sign of $\lambda$ they may be real/complex.

2) Let us consider the anzats with factorized function $F(\vec{x}), F=F_{+}\left(x_{+}\right) \cdot F_{-}\left(x_{-}\right)$. Then from Eq.(215),

$$
C_{ \pm}=\frac{\nu_{ \pm}}{F_{ \pm}} \pm \frac{\gamma}{F_{ \pm}} \int^{x_{ \pm}} F_{ \pm} d x_{ \pm}^{\prime}
$$

and there are two options to fulfill the condition (216), i.e. $F(\vec{x})=F_{1}\left(2 x_{1}\right)+F_{2}\left(2 x_{2}\right)$,
a) $\quad F_{ \pm}\left(x_{ \pm}\right)=\epsilon_{ \pm} x_{ \pm}$,
b) $F_{ \pm}=\sigma_{ \pm} \exp \left(\sqrt{\lambda} \cdot x_{ \pm}\right)+\delta_{ \pm} \exp \left(-\sqrt{\lambda} \cdot x_{ \pm}\right)$.

Corresponding potentials can be found according to Eq.(218), being similar to ones obtained in [155] in quite different approach.

3) Let us start now from the general solution of (215),

$$
F=L\left(\int \frac{d x_{+}}{C_{+}}-\int \frac{d x_{-}}{C_{-}}\right) /\left(C_{+} C_{-}\right) .
$$

Then Eq.(216) gives the functional-differential equation for the functional $L\left(A_{+}-A_{-}\right)$ with $A_{ \pm}^{\prime} \equiv 1 / C_{ \pm}\left(x_{ \pm}\right)$,

$$
\left(\frac{A_{+}^{\prime \prime \prime}}{A_{+}^{\prime}}-\frac{A_{-}^{\prime \prime \prime}}{A_{-}^{\prime}}\right) L\left(A_{+}-A_{-}\right)+3\left(A_{+}^{\prime \prime}+A_{-}^{\prime \prime}\right) L^{\prime}\left(A_{+}-A_{-}\right)+\left(A_{+}^{\prime 2}-A_{-}^{2}\right) L^{\prime \prime}\left(A_{+}-A_{-}\right)=0,
$$

where $L^{\prime}$ denotes the derivative of $L$ with respect to its argument. If we take functions $A_{ \pm}$such that $A_{ \pm}^{\prime \prime}=\lambda^{2} A_{ \pm}, \lambda=$ const, Eq.(222) will become ordinary differential equation for $L$ with independent variable $\left(A_{+}-A_{-}\right)$. It can be easily solved,

$$
L\left(A_{+}-A_{-}\right)=\alpha\left(A_{+}-A_{-}\right)^{-2}+\beta,
$$


where $A_{ \pm}=\sigma_{ \pm} \exp \left(\lambda x_{ \pm}\right)+\delta_{ \pm} \exp \left(-\lambda x_{ \pm}\right)$with $\sigma_{+} \cdot \delta_{+}=\sigma_{-} \cdot \delta_{-}$and $\alpha, \beta$ - real constants. For $\lambda^{2}>0$, choosing $\sigma_{ \pm}=-\delta_{ \pm}=k / 2$ or $\sigma_{ \pm}=+\delta_{ \pm}=k / 2$, we obtain (up to an arbitrary shift in $x_{ \pm}$) two particular solutions,

$$
\text { 3a) } \left.\quad A_{ \pm}=k \sinh \left(\lambda x_{ \pm}\right), \quad 3 b\right) \quad A_{ \pm}=k \cosh \left(\lambda x_{ \pm}\right) .
$$

Then (221) leads to,

3a) $\quad F_{1}(2 x)=\frac{k_{1}}{\cosh ^{2}(\lambda x)}+k_{2} \cosh (2 \lambda x)$;

$$
F_{2}(2 x)=\frac{k_{1}}{\sinh ^{2}(\lambda x)}+k_{2} \cosh (2 \lambda x) ; \quad C_{ \pm}=\frac{k}{\cosh \left(\lambda x_{ \pm}\right)}, \quad k \neq 0,
$$

3b) $\quad F_{1}(2 x)=-F_{2}(2 x)=\frac{k_{1}}{\sinh ^{2}(\lambda x)}+k_{2} \sinh ^{2}(\lambda x), \quad C_{ \pm}=\frac{k}{\sinh \left(\lambda x_{ \pm}\right)}, \quad k \neq 0$.

For $\lambda^{2}<0$ hyperbolic functions must be substituted by trigonometric ones.

We have to remark that the case $\lambda^{2}=0$, i.e. $A_{ \pm}^{\prime \prime}=0$, is not of interest, leading to trivial superpartners. However, choosing in (224) $\lambda \rightarrow 0, k, k_{1}, k_{2}^{-1} \rightarrow 0$ simultaneously, so that $\lambda^{2} \sim k_{1} \sim k_{2}^{-1} \sim k^{2}$, we obtain the solution,

$$
F_{1}(2 x)=-F_{2}(2 x)=\tilde{k}_{1} x^{-2}+\tilde{k}_{2} x^{2}, \quad C_{ \pm}=\frac{\tilde{k}}{x_{ \pm}} .
$$

One can check that (215) is also satisfied by

$$
F_{1}(2 x)=-F_{2}(2 x)=k_{1} x^{2}+k_{2} x^{4}, \quad C_{ \pm}= \pm \frac{k}{x_{ \pm}} .
$$

4) Starting again from (221), it is convenient to pass on to new variable functions $C_{ \pm} \equiv \pm f_{+} / f_{ \pm}^{\prime}$. Then $F$ in (221) is represented in the form $F=U\left(f_{+} f_{-}\right) f_{+}^{\prime} f_{-}^{\prime}$ with an arbitrary $\llbracket$ function $U$. After substitution in (216) one obtains the functional-differential equation,

$$
\left(f_{+}^{\prime 2} f_{-}^{2}-f_{+}^{2} f_{-}^{\prime 2}\right) U^{\prime \prime}(f)+3 f\left(\frac{f_{+}^{\prime \prime}}{f_{+}}-\frac{f_{-}^{\prime \prime}}{f_{-}}\right) U^{\prime}(f)+\left(\frac{f_{+}^{\prime \prime \prime}}{f_{+}^{\prime}}-\frac{f_{-}^{\prime \prime \prime}}{f_{-}^{\prime}}\right) U(f)=0, \quad f \equiv f_{+} f_{-} .
$$

For particular form of functions $f_{ \pm}=\alpha_{ \pm} \exp \left(\lambda x_{ \pm}\right)+\beta_{ \pm} \exp \left(-\lambda x_{ \pm}\right)$, this equation becomes an ordinary differential equation for $U$ with independent variable $f$. Its solution is $U=a+4 b f_{+} f_{-}(a, b-$ real constants $)$. Then functions

$$
\begin{aligned}
F_{1}(x)= & k_{1}\left(\alpha_{+} \alpha_{-} \exp (\lambda x)+\beta_{+} \beta_{-} \exp (-\lambda x)\right) \\
& +k_{2}\left(\alpha_{+}^{2} \alpha_{-}^{2} \exp (2 \lambda x)+\beta_{+}^{2} \beta_{-}^{2} \exp (-2 \lambda x)\right), \\
-F_{2}(x) & =k_{1}\left(\alpha_{+} \beta_{-} \exp (\lambda x)+\beta_{+} \alpha_{-} \exp (-\lambda x)\right) \\
& +k_{2}\left(\alpha_{+}^{2} \beta_{-}^{2} \exp (2 \lambda x)+\beta_{+}^{2} \alpha_{-}^{2} \exp (-2 \lambda x)\right), \\
C_{ \pm}= \pm & \frac{\alpha_{ \pm} \exp \left(\lambda x_{ \pm}\right)+\beta_{ \pm} \exp \left(-\lambda x_{ \pm}\right)}{\lambda\left(\alpha_{ \pm} \exp \left(\lambda x_{ \pm}\right)-\beta_{ \pm} \exp \left(-\lambda x_{ \pm}\right)\right)}
\end{aligned}
$$

(with $k_{1} \equiv a \lambda^{2}, k_{2} \equiv 4 b \lambda^{2}$ ) are real solutions of (215), (216), if $\alpha_{ \pm}, \beta_{ \pm}$are real for $\lambda^{2}>0$, and $\alpha_{ \pm}=\beta_{ \pm}^{*}$ for $\lambda^{2}<0$.

$\|$ Due to Eq.(216), the function $F$ should be additionally representable in the form $F=F_{1}\left(2 x_{1}\right)+$ $F_{2}\left(2 x_{2}\right)$. 
5) To find a next class of solutions it is useful to rewrite (215) in terms of $x_{1,2}$, $\left(F_{1}\left(2 x_{1}\right)+F_{2}\left(2 x_{2}\right)\right) \partial_{1}\left(C_{+}+C_{-}\right)+F_{1}^{\prime}\left(2 x_{1}\right)\left(C_{+}+C_{-}\right)+F_{2}^{\prime}\left(2 x_{2}\right)\left(C_{+}-C_{-}\right)=0$.

Among known particular solutions the most compact one is,

$$
C_{+}(x)=C_{-}(x)=a x^{2}+c, \quad F_{1}\left(2 x_{1}\right)=0, \quad F_{2}\left(2 x_{2}\right)=\frac{b^{2}}{x_{2}^{2}} .
$$

After inserting these solutions (223) - (228) into the general formulas (218), one obtains the analytical expressions for potentials. Their explicit form can be found in [91].

Two additional classes of particular solutions of the system (204) - (206) were obtained analogously by means of suitable ansatzes for the case of degenerate metrics $g_{i k}=\operatorname{diag}(1,0)\left(\right.$ see [91]) and the case of deformed hyperbolic metrics $g_{i k}=\operatorname{diag}\left(1,-a^{2}\right)$ with $a \neq 0$ (see [98]).

\subsection{Integrability}

Similarly to the case of one-dimensional Polynomial SUSY Quantum Mechanics (Section 3), partner two-dimensional Hamiltonians $h^{(0)}$ and $h^{(1)}$ can be considered as components of the diagonal super-Hamiltonian $H$, which are intertwined by components $q^{ \pm}$of the off-diagonal supercharges $Q^{ \pm}$. Thus, the operators $H, Q^{ \pm}$constitute the deformed SUSY algebra: both supercharges are nilpotent operators, and they commute with the superHamiltonian. The difference is in the third relation of SUSY algebra: while in onedimensional case anticommutator of $Q^{ \pm}$produced the second order polynomial of $H$, in two-dimensional situation it gives some fourth order diagonal operator [88, [89], [90],

$$
\left\{Q^{+}, Q^{-}\right\}=\hat{R} ; \quad \hat{R}=\left(\begin{array}{cc}
R^{(0)} & 0 \\
0 & R^{(1)}
\end{array}\right)=\left(\begin{array}{cc}
q^{+} q^{-} & 0 \\
0 & q^{-} q^{+}
\end{array}\right) .
$$

In general, $R^{(0)}, R^{(1)}$ are not expressed in terms of $h^{(0)}, h^{(1)}$ in this case, but nevertheless they are related. Indeed, as it follows directly from the intertwining relations (197), they are the fourth order symmetry operators of corresponding systems,

$$
\left[h^{(0)}, R^{(0)}\right]=\left[h^{(1)}, R^{(1)}\right]=0 .
$$

In general, these symmetry operators might be expressed as functions of $h^{(i)}, i=0,1$, similarly to the one-dimensional case. But investigation [89], [91], [90] shows that this is not the case: two opportunities can be realized depending on the metrics $g_{i k}$ in the supercharges.

1) For the case of elliptic (Laplacian) metrics $g_{i k}=\delta_{i k}$ (and only for this one), the fourth order operators $R^{(i)}, i=0,1$ are expressed in terms of Hamiltonians $h^{(i)}$ and the second order operators $\widetilde{R}^{(i)}$, which also commute with $h^{(i)}$,

$$
R^{(i)}=\left(h^{(i)}\right)^{2}+\eta h^{(i)}+\widetilde{R}^{(i)}, \quad\left[h^{(i)}, \widetilde{R}^{(i)}\right]=0
$$

with $\eta=$ Const. The explicit forms of differential operators $R^{(i)}$ and of corresponding Hamiltonians $h^{(i)}$ depend on the values of constants in (207), and they are given in 
[89], [90]. It was shown that these Hamiltonians allow the standard procedure of $R$-separation of variables [153] in polar, parabolic or elliptic coordinates.

2) For all other allowed metrics (199), the fourth order operators $R^{(i)}, i=0,1$ can not be reduced to any symmetry operators of lower order. This was shown in the general form in [89], and it follows also from the results of L.P.Eisenhart [156], where the exhaustive list of systems which are amenable to separation of variables was given (just in Cartesian, polar, elliptic and parabolic coordinates).

Thus, for all possible metrics (199) each member of the variety of two-dimensional Hamiltonians $h^{(i)}$, which satisfy the intertwining relations (197), has the symmetry operator $R^{(i)}$, and therefore is completely integrable. In general, this fact does not provide the solvability of the models, but some of them are partially or completely solvable (see the next Section).

In papers [90], [91], the classical limit of Polynomial two-dimensional SUSY QM was considered. In particular, the prescription how to look for the integrals of motion (classical analogues of symmetry operators) of fourth order in momenta for given classical Hamiltonian was formulated. Namely, it is necessary to find such the complex classical functions $q_{c l}^{+}(\vec{x}, \vec{p})=\left(q_{c l}^{-}(\vec{x}, \vec{p})\right)^{\dagger}$ in the phase space, that they satisfy the following relations:

$$
\left\{q_{c l}^{+}, h_{c l}\right\}=i f(\vec{x}) q_{c l}^{+} ; \quad\left\{q_{c l}^{-}, h_{c l}\right\}=-i f(\vec{x}) q_{c l}^{-},
$$

where $\{\cdot, \cdot\}$ are Poisson brackets, and $f(\vec{x})-$ an arbitrary real function of $\vec{x}$. If such real function exists, the classical Hamiltonian $h_{c l}$ obeys the factorizable integral of motion $I_{c l}=q_{c l}^{+} q_{c l}^{-}$of fourth order in momenta,

$$
\left\{h_{c l}, I_{c l}\right\}=0 .
$$

We see that the identification of classical integral of motion from the quantum SUSY algebra is unambiguous. However the opposite is not always true: a quantum anomaly may arise [106].

\section{SUSY separation of variables for $d=2$}

The idea of shape invariance was very productive in one-dimensional SUSY Quantum Mechanics (see Section 6 and references therein). In the present Section, the twodimensional analogue of that shape invariance will be exploited effectively [92, 994, [13], 195], 100, [16], 102], [103], 104, [105] for two-dimensional Hamiltonians which satisfy the supersymmetrical intertwining relations (197). In contrast to one-dimensional case, there are essential differences in $d=2$ : in general, the ground state of $h^{(1)}(a)$ does not coincide with zero modes of second order supercharge $q^{+}(a)$, the value $E_{0}(a) \neq 0$, and also many zero modes of $q^{+}$exist. Up to these crucial differences, all other actions can be repeated. It was shown [92], [94], [13], [105] that such direct generalization of the procedure above may provide only partial (quasi-exact) solvability of the model (see the Subsection 5.2). But for some models [100, [102], [16], [104], [106], [115] it will help to solve the model completely (see Subsection 5.3). 
The idea to consider the quasi-exactly-solvable models - the intermediate class between exactly solvable and unsolvable analytically models - was introduced on 80ties in [157] - 163]. In particular, the series of papers by A.Turbiner, A.Ushveridze and M.Shifman was devoted to the elegant algebraic method of construction of one-dimensional quasi-exactly-solvable (and sometimes, of exactly solvable) quantum models. In general, this method works beyond the supersymmetry, but both approaches can be combined in one-dimensional case as in [164]. This approach is applicable to twodimensional problems as well, but only in curved spaces with nontrivial metrics [161].

The realization of such opportunities to solve non-trivial two-dimensional models partially, or even completely, would be very important since only one regular method to solve analytically the Schrödinger equation for two-dimensional models is known: reduction to a pair of one-dimensional problems by means of the procedure of separation of variables [153]. This method can be used for very restrictive class of models, and full classification of models which allowed separation of variables was given by L.P.Eisenhart [156]: four possibilities exist - Cartesian, polar, elliptic and parabolic coordinates. The general form of potentials amenable to separation of variables is also known explicitly up to arbitrary functions of one variable. And analytical solution is possible only if these functions belong to the list of exactly solvable one-dimensional potentials. All these Hamiltonians $H$ are integrable: the symmetry operator $R$ of second order in derivatives (in momenta) exists, $[H, R]=0$. Besides models amenable to separation of variables, the class of so called Calogero-like models [165], [18] is known as well. They describe the specific forms of pairwise interaction of $N$ particles on a line, and they are solvable by means of special transformation of variables which leads to a separation of variables. Recently, new classes of solvable two-dimensional models were built in [166], [167], [168], but all these models are superintegrable and amenable to separation of variables.

In two following Subsections, we shall present two special procedures of SUSYseparation of variables in two-dimensional models, which do not allow to use the conventional separation of variables. Subsection 9.2 presents the first procedure of SUSY separation of variables where variables are separated in the supercharge. It leads to QES models, and the specific model of two-dimensional Morse potential illustrates this method. In Subsection 9.3 the second procedure of SUSY separation of variables is given where variables are separated in one of partner Hamiltonians. In the case of the same Morse model, but with particular values of parameter, it allows to solve the model completely, i.e. to find analytically the whole spectrum and all wave functions.

\subsection{SUSY-separation of variables I: QES models}

The first variant of SUSY-separation of variables is realized when the Hamiltonians $h^{(0)}, h^{(1)}$ do not allow standard separation of variables, but the supercharge $q^{+}$does allow [92], [13], 94], [97], 99], [16], [105]. The general scheme is the following. Let's suppose that we know zero modes of $q^{+}$,

$$
q^{+} \Omega_{n}(\vec{x})=0 ; \quad n=0,1, \ldots, N ; \quad q^{+} \vec{\Omega}(\vec{x})=0 .
$$


Due to intertwining relations (197), the Hamiltonian $h^{(1)}$ obey the important property: the space of zero modes $\Omega_{n}$ is closed under the action of $h^{(1)}$,

$$
h^{(1)} \vec{\Omega}(\vec{x})=\hat{C} \vec{\Omega}(\vec{x})
$$

with constant matrix $\hat{C}$. If this matrix is known, and if it can be diagonalized,

$$
\hat{B} \hat{C}=\hat{\Lambda} \hat{B} ; \quad \hat{\Lambda}=\operatorname{diag}\left(\lambda_{0}, \lambda_{1}, \ldots, \lambda_{N}\right),
$$

the eigenvalues of $h^{(1)}$ can be found algebraically,

$$
h^{(1)}(\hat{B} \vec{\Omega}(\vec{x}))=\hat{\Lambda}(\hat{B} \vec{\Omega}(\vec{x})) .
$$

Thus, to realize this scheme of construction some number of energy values and corresponding wave functions, we need:

- to find zero modes $\Omega_{n}(\vec{x})$;

- to find constant matrix $B$, such that $\hat{B} \hat{C}=\hat{\Lambda} \hat{B}$.

As for the first step, the zero modes can be obtained by using the special similarity transformation (not unitary!), which removes the terms linear in derivatives from $q^{+}$,

$$
\begin{gathered}
\tilde{q}^{+}=e^{-\chi(\vec{x})} q^{+} e^{+\chi(\vec{x})}=\partial_{1}^{2}-\partial_{2}^{2}+\frac{1}{4}\left(F_{1}\left(2 x_{1}\right)+F_{2}\left(2 x_{2}\right)\right) ; \\
\chi(\vec{x})=-\frac{1}{4}\left(\int C_{+}\left(x_{+}\right) d x_{+}+\int C_{-}\left(x_{-}\right) d x_{-}\right) .
\end{gathered}
$$

Now, $\tilde{q}^{+}$allows separation of variables for arbitrary solution of intertwining relations, and we obtain the first variant of new procedure - SUSY-separation of variables. Similarly to the conventional separation of variables, separation of variables in the operator $\tilde{q}^{+}$itself does not guarantee solvability of the problem.

The next task is to solve two one-dimensional problems,

$$
\begin{aligned}
& \left(-\partial_{1}^{2}-\frac{1}{4} F_{1}\left(2 x_{1}\right)\right) \eta_{n}\left(x_{1}\right)=\epsilon_{n} \eta_{n}\left(x_{1}\right) ; \\
& \left(-\partial_{2}^{2}+\frac{1}{4} F_{2}\left(2 x_{2}\right)\right) \rho_{n}\left(x_{2}\right)=\epsilon_{n} \rho_{n}\left(x_{2}\right) .
\end{aligned}
$$

Three remarks are appropriate now.

Remark 1: the same similarity transformation of $h^{(1)}$ does not lead to operator amenable to separation of variables.

Remark 2: the normalizability of $\Omega_{n}$ has to be studied attentively due to nonunitarity of the similarity transformation.

Remark 3: we have no reasons to expect exact solvability of the model, but quasiexact-solvability can be predicted.

As for the matrix $\hat{B}$, it must be found by some specific procedure. Such procedure was used in example which will be presented below.

In principle, the first scheme of $S U S Y$ - separation of variables can be used for arbitrary models satisfying intertwining relations by supercharges with Lorentz metrics. 
The list of solutions of intertwining relations is already rather long (see Subsection 8.2.2), and it may increase in future. The main obstacle is analytical solvability of one-dimensional equations, obtained after separation of variables in the operator $\tilde{q}^{+}$.

Below we describe briefly such a model which can be considered as the generalized two-dimensional Morse potential,

$$
\begin{aligned}
& C_{+}=4 a \alpha ; \quad C_{-}=4 a \alpha \cdot \operatorname{coth} \frac{\alpha x_{-}}{2} ; \\
& f_{i}\left(x_{i}\right) \equiv \frac{1}{4} F_{i}\left(2 x_{i}\right)=-A\left(e^{-2 \alpha x_{i}}-2 e^{-\alpha x_{i}}\right) ; \quad i=1,2 \\
& V^{(0),(1)}= \alpha^{2} a(2 a \mp 1) \sinh ^{-2}\left(\frac{\alpha x_{-}}{2}\right)+4 a^{2} \alpha^{2} \\
&+A\left[e^{-2 \alpha x_{1}}-2 e^{-\alpha x_{1}}+e^{-2 \alpha x_{2}}-2 e^{-\alpha x_{2}}\right] ;
\end{aligned}
$$

where $A>0, \alpha>0, a-$ real.

To explain the name, we present the potentials in the form,

$$
V(\vec{x})=V_{\text {Morse }}\left(x_{1}\right)+V_{\text {Morse }}\left(x_{2}\right)+v\left(x_{1}, x_{2}\right),
$$

where first two terms are just one-dimensional Morse potentials, and the last term mixes variables $x_{1}, x_{2}$.

The solutions of one-dimensional Schrödinger equations with Morse potentials $V_{\text {Morse }}(x)$ are well known [169], and the zero modes can be written [92, [13] as,

$$
\begin{aligned}
& \Omega_{n}(\vec{x})=\left(\frac{\alpha}{\sqrt{A}} \cdot \frac{\xi_{1} \xi_{2}}{\left|\xi_{2}-\xi_{1}\right|}\right)^{2 a} \exp \left(-\frac{\xi_{1}+\xi_{2}}{2}\right)\left(\xi_{1} \xi_{2}\right)^{s_{n}} \cdot F\left(-n, 2 s_{n}+1 ; \xi_{1}\right) F\left(-n, 2 s_{n}+1 ; \xi_{2}\right) \\
& \xi_{i} \equiv \frac{2 \sqrt{A}}{\alpha} \exp \left(-\alpha x_{i}\right) ; \quad s_{n}=\frac{\sqrt{A}}{\alpha}-n-\frac{1}{2}>0
\end{aligned}
$$

The conditions of normalizability and of absence of the "fall to the center" are,

$$
a \in\left(-\infty,-\frac{1}{4}-\frac{1}{4 \sqrt{2}}\right) ; \quad s_{n}=\frac{\sqrt{A}}{\alpha}-n-\frac{1}{2}>-2 a>0
$$

To obtain the matrix $\hat{C}$ explicitly, one must act by $h^{(1)}$ on $\Omega_{n}$. The matrix turns out to be triangular, and therefore, the energy eigenvalues coincide with its diagonal elements,

$$
E_{k}=c_{k k}=-2\left(2 a \alpha^{2} s_{k}-\epsilon_{k}\right) .
$$

To find a variety of wave functions is a more difficult task. For that it is necessary to find all elements of $\hat{C}$ and all elements of matrix $\hat{B}$. The recurrent procedure for the case of two-dimensional Morse potential was given in [92, [13. This variety can be enlarged by means of shape invariance property (128) of the model, which can be easily checked. Similarly to one-dimensional shape invariance, each wave function constructed by SUSY-separation of variables leads to a set of additional wave functions, which can 
be written as,

$$
\begin{aligned}
& h^{(0)}(a)\left[q^{-}(a) q^{-}\left(a-\frac{1}{2}\right) \ldots q^{-}\left(a-\frac{M-1}{2}\right) \Psi\left(a-\frac{M}{2}\right)\right]= \\
& \left(E_{0}\left(a-\frac{M}{2}\right)+\mathcal{R}\left(a-\frac{M-1}{2}\right)+\ldots+\mathcal{R}(a)\right) \\
& \times\left[q^{-}(a) q^{-}\left(a-\frac{1}{2}\right) \ldots q^{-}\left(a-\frac{M-1}{2}\right) \Psi\left(a-\frac{M}{2}\right)\right]
\end{aligned}
$$

with integer $M$. Analogous approach works for the two-dimensional generalization of Pöschl-Teller model [94], for some two-dimensional periodic potentials [99] and for the two-dimensional generalization of Scarf II potential [105].

\subsection{SUSY-separation of variables II: Exact solvability.}

Among all known solutions of two-dimensional intertwining relations with second order supercharges a subclass exists [100], [102, [96], [16], [104], where one of intertwined Hamiltonians $h^{(1)}$ is amenable to standard separation of variables due to specific choice of parameters of the model. Its superpartner $h^{(0)}$ still does not allow conventional separation of variables.

The scheme will be described below for the same specific model - two-dimensional generalization of Morse potential,

$$
V^{(0),(1)}=\alpha^{2} a(2 a \mp 1) \sinh ^{-2}\left(\frac{\alpha x_{-}}{2}\right)+4 a^{2} \alpha^{2}+A\left[e^{-2 \alpha x_{1}}-2 e^{-\alpha x_{1}}+e^{-2 \alpha x_{2}}-2 e^{-\alpha x_{2}}\right]
$$

Let's choose $a_{0}=-1 / 2$ in order to vanish the mixed term in $V^{(1)}$, and therefore, the Hamiltonian $h^{(1)}$ allows the conventional separation of variables. Moreover, after separation of variables each of obtained one-dimensional problems is again exactly solvable: they coincide with one-dimensional problems of the previous Subsection, where they occured in a different context (separation of variables in $\tilde{q}$ ).

The discrete spectrum of this one-dimensional model is,

$$
\epsilon_{n}=-\alpha^{2} s_{n}^{2} ; \quad s_{n} \equiv \frac{\sqrt{A}}{\alpha}-n-\frac{1}{2}>0 ; \quad n=0,1,2, \ldots,
$$

Wave functions are expressed in terms of confluent hypergeometric functions,

$$
\eta_{n}\left(x_{i}\right)=\exp \left(-\frac{\xi_{i}}{2}\right)\left(\xi_{i}\right)^{s_{n}} F\left(-n, 2 s_{n}+1 ; \xi_{i}\right) ; \quad \xi_{i} \equiv \frac{2 \sqrt{A}}{\alpha} \exp \left(-\alpha x_{i}\right) .
$$

Due to separation of variables, the two-dimensional problem with $h^{(1)}(\vec{x})$ is exactly solvable. Its energy eigenvalues are,

$$
E_{n, m}=E_{m, n}=\epsilon_{n}+\epsilon_{m},
$$

being two-fold degenerate for $n \neq m$. The corresponding eigenfunctions can be chosen as symmetric or (for $n \neq m$ ) antisymmetric combinations,

$$
\Psi_{E_{n, m}}^{(1) S, A}(\vec{x})=\eta_{n}\left(x_{1}\right) \eta_{m}\left(x_{2}\right) \pm \eta_{m}\left(x_{1}\right) \eta_{n}\left(x_{2}\right) .
$$


Our initial aim here is to solve completely the problem for $h^{(0)}(\vec{x})$ with $a_{0}=-1 / 2$. The main tool is again the SUSY intertwining relations, i.e. isospectrality of $h^{(0)}$ and $h^{(1)}$ but up to zero modes and singular properties of $q^{ \pm}$. In general, we may expect three kinds of levels of $h^{(0)}(\vec{x})$,

(i). The levels, which coincide with $E_{n m}$. Their wave functions can be obtained from $\Psi^{(1)}$ by means of $q^{+}$.

(ii). The levels, which were absent in the spectrum of $h^{(1)}(\vec{x})$, if some wave functions of $h^{(0)}(\vec{x})$ are simultaneously the zero modes of $q^{-}$. Then the second intertwining relation would not give any partner state among bound states of $h^{(1)}(\vec{x})$.

(iii). The levels, which were also absent in the spectrum of $h^{(1)}(\vec{x})$, if some wave functions of $h^{(0)}(\vec{x})$ become nonnormalizable after action of operator $q^{-}$.

We have to analyze these three classes of possible bound states of $h^{(0)}$ one after another.

(i). The first SUSY intertwining relation gives the two-fold degenerate wave functions of $h^{(0)}$ with energies $E_{n m}, \Psi_{E_{n m}}^{(0)}=q^{+} \Psi_{E_{n m}}^{(1)}$. But $q^{+}$includes singularity on the line $x_{1}=x_{2}$, therefore the normalizability of $\Psi_{E_{n, m}}^{(0)}$ depends crucially on the behavior of $\Psi_{E_{n, m}}^{(1)}$ on the line $\xi_{1}=\xi_{2}$. One can check that only antisymmetric functions $\Psi^{(1)}$ survive, i.e. only symmetric $\Psi^{(0)}$ survive. This fact can be demonstrated [100] both by direct calculation and by indirect method - by means of symmetry operator $R^{(0)}$.

The indirect method explores that the symmetry operator $R^{(0)}=q^{-} q^{+}$for $a_{0}=-1 / 2$ can be written in terms of one-dimensional Morse parts $h_{1}\left(x_{1}\right), h_{2}\left(x_{2}\right)$ of the Hamiltonian $h^{(1)}=h_{1}\left(x_{1}\right)+h_{2}\left(x_{2}\right)$,

$$
R^{(0)}=\left(h_{1}\left(x_{1}\right)-h_{2}\left(x_{2}\right)\right)^{2}+2 \alpha^{2}\left(h_{1}\left(x_{1}\right)+h_{2}\left(x_{2}\right)\right)+\alpha^{4} .
$$

Therefore,

$$
R^{(0)} \Psi_{E_{n, m}}^{(0) A}(\vec{x})=r_{n, m} \Psi_{E_{n, m}}^{A}(\vec{x}) ; r_{n, m}=\alpha^{4}\left[(n-m)^{2}-1\right]\left[\left(s_{n}+s_{m}\right)^{2}-1\right],
$$

and

$$
\left\|\Psi_{E_{n, m}}^{(1) S}\right\|^{2}=\left\langle\Psi_{E_{n, m}}^{(0) A}\left|q^{-} q^{+}\right| \Psi_{E_{n, m}}^{(0) A}\right\rangle=r_{n, m}\left\|\Psi_{E_{n, m}}^{(0) A}\right\|^{2} .
$$

For $n=m$, wave functions $\Psi_{E_{n, n}}^{(0) S}$ vanish identically by trivial reasons. It is clear now that wave functions $\Psi_{E_{n, n \pm 1}}^{(0) S}$ also vanish. For all other $n, m$, functions $\Psi_{E_{n, m}}^{(0) S}$ have positive and finite norm, and there is no degeneracy of these levels.

(ii). These possible bound states of $h^{(0)}$ are the normalizable zero modes of $q^{-}$. The variety of such zero modes is known from [92]: they exist only for positive values of $a$

$$
a \in\left(\frac{1}{4}+\frac{1}{4 \sqrt{2}},+\infty\right),
$$

which does not contain the value $a_{0}=-1 / 2$. Thus, no normalizable bound states of this class exist for $h^{(0)}$.

(iii). We have to study an opportunity that $q^{-}$destroys normalizability of some eigenfunctions of $h^{(0)}$. It could occur due to singular character of $q^{-}$at $x_{1}=x_{2}$. The 
analysis was performed [100] in suitable coordinates. It shows that $q^{-}$is not able to transform normalizable wave function to nonnormalizable. Therefore, the third class of possible wave functions $h^{(0)}$ does not exist too.

Summing up, the spectrum of $h^{(0)}$ with $a_{0}=-1 / 2$ consists only of the bound states with energies $E_{n m}$ for $|n-m|>1$. This spectrum is bounded from above by the condition of positivity of $s_{n}, s_{m}: n, m<\sqrt{A} / \alpha-1 / 2$. The corresponding wave functions are obtained analytically [100].

The results above can be expanded to the whole hierarchy of Morse potentials with $a_{k}=-(k+1) / 2$ with $k=0,1, \ldots$ by means of shape invariance property. Let's denote elements of the hierarchy as $h^{(0)}\left(\vec{x} ; a_{k}\right), h^{(1)}\left(\vec{x} ; a_{k}\right)$. All these Hamiltonians are also exactly solvable due to shape invariance of the model,

$$
h^{(0)}\left(\vec{x} ; a_{k-1}\right)=h^{(1)}\left(\vec{x} ; a_{k}\right) ; k=1,2, \ldots
$$

This means that the following chain (hierarchy) of Hamiltonians can be built,

$h^{(1)}\left(\vec{x} ; a_{0}\right) \div h^{(0)}\left(\vec{x} ; a_{0}\right)=h^{(1)}\left(\vec{x} ; a_{1}\right) \div h^{(0)}\left(\vec{x} ; a_{1}\right)=\ldots \div h^{(1)}\left(\vec{x} ; a_{k-1}\right)=h^{(0)}\left(\vec{x} ; a_{k}\right) \div h^{(0)}\left(\vec{x} ; a_{k}\right)$,

where the sign $\div$ denotes intertwining by $q^{ \pm}\left(a_{i}\right)$.

In the general case, the functions

$\Psi_{E_{n, m}}^{(0)}\left(\vec{x} ; a_{k}\right)=q^{+}\left(a_{k}\right) \Psi_{E_{n, m}}^{(1)}\left(\vec{x} ; a_{k}\right)=q^{+}\left(a_{k}\right) q^{+}\left(a_{k-1}\right) \ldots q^{+}\left(a_{0}\right) \Psi_{E_{n, m}}^{(1) A}\left(\vec{x} ; a_{0}\right)$

(if normalizable) are the wave functions of $h^{(0)}\left(\vec{x} ; a_{k}\right)$ with energies $E_{n, m}=-\alpha^{2}\left(s_{n}^{2}+s_{m}^{2}\right)$. The symmetries of wave functions alternate and depend on the length of chain. This is true but up to zero modes of operators $q^{+}$.

It is necessary to keep under the control normalizability of $\Psi$ and zero modes of $q^{+}$. This control is performed algebraically by means of identity, which must be fulfilled up to a function of $H$,

$$
R^{(1)}\left(a_{k}\right)=R^{(0)}\left(a_{k-1}\right) .
$$

Actually, the following equation holds,

$q^{-}\left(a_{k}\right) q^{+}\left(a_{k}\right)=q^{+}\left(a_{k-1}\right) q^{-}\left(a_{k-1}\right)+\alpha^{2}(2 k+1)\left[2 h^{(0)}\left(\vec{x} ; a_{k-1}\right)+\alpha^{2}\left(2 k^{2}+2 k+1\right)\right]$.

These relations allowed to evaluate the norms of wave functions. The result is the following. The spectra of Hamiltonians $h^{(0)}\left(\vec{x} ; a_{k}\right)$ are not degenerate. They consist of the bound states with energies $E_{n, m}$, with indices $|n-m|>k+2$, and their wave functions $\Psi_{E_{n, m}}^{(0)}\left(\vec{x} ; a_{k}\right)$ were given analytically above.

The procedure of this Subsection was reproduced fully for two other twodimensional models which are not amenable to standard separation: generalized PöschlTeller [102] and Scarf II [104] potentials. The full energy spectra and corresponding wave functions were also built analytically by the second variant of SUSY-separation of variables. 


\section{Perspectives, some applications and missing points}

The purpose of this review has been to elucidate the recent progress in Nonlinear SUSY realization by intertwining Darboux transformations for a community working on methods of isospectral design and their applications. We wanted also to draw an attention to new (quasi-)exactly solvable potential systems in two dimensions nonseparable by a conventional choice of coordinates. Summarizing our experience we describe the general SUSY QM as governed by the extended nonlinear SUSY algebra

with $\mathcal{N}$ pairs of nilpotent supercharges $Q_{j}, \bar{Q}_{j}=Q_{j}^{t}=Q_{j}^{\dagger}$ and a number of hermitian hidden-symmetry differential operators $R_{\alpha}=R_{\alpha}^{\dagger}, \quad\left[R_{\alpha}, R_{\beta}\right]=0 ; \quad 0 \leq \alpha, \beta \leq M$.

Not all the research areas that are linked to the subject of the present review have been covered in the main text. A number of extensions and applications of Nonlinear SUSY QM have been found and still there remains a plenty of open questions and challenges to be solved. Below we list some of these directions and mention few missing topics.

- The method of multidimensional supersymmetric intertwining relations and shape invariance turned out to be useful for study of different variants of Calogero-like models of $N$ particles on a line, including the models with internal degrees of freedom and with pairwise interactions based on any root systems [108, [170][177] (see the recent review [18]).

- One-dimensional supersymmetric models with matrix superpotentials and supercharges [178] - 184 have been used either for description of motion of spin particles in external fields [69] - [71], [110, [114], [185] - [190] or to study scattering of particles with strong coupling of channels [185],[186], [191] - [194] with practical application of the results to specific processes or to investigate spectral problem for the Bogoliubov-de Gennes system [196]. Such systems are not fully investigated in searching of extended SUSY systems with hidden symmetries. It is clear that a comprehensive understanding of irreducible building blocks for spectral design analogously to the scalar case is not yet achieved. Also, matrix models with higher dimensionality of space have to be studied. To start with, two-dimensional models with matrix superpotential were studied in [195].

- A natural question appears in respect to the classical formulation of nonlinear SUSY whether a canonical quantization scheme similar to that of Subsect.2.3 exists in this case. A corresponding pseudoclassical Lagrangian for the particular one-dimensional model was observed in [132 and investigated in a more general setting in [106], 253]. It was found that quantization of one-dimensional nonlinear supersymmetry faces a problem of the quantum anomaly. Thus the problem arises to identify quantum anomaly in the two- and higher dimensional case. On the other hand the anomaly mentioned above is originated from noncommutativity of momenta and coordinates (ordering problem) which is unambiguously resolved in our recipe of supercharge identification and therefore does not affect any of the results presented in the review. 
- Coherent states in the framework of SUSY QM models were built in [197] - [200], but only for one-dimensional systems [201].

- Formulation of multidimensional SUSY QM in arbitrary curvilinear coordinates was developed in [202] (see also [203] - 205]). This formalism could be useful for investigation of SUSY design of different cosmological and brain world models.

- Generalization of Polynomial SUSY QM for higher (first of all, $d=3$ ) space dimensions seems to be very interesting (see [101]).

- Effective mass SUSY QM is a rather popular generalization of conventional SUSY QM for the Schrödinger equation with coordinate-dependent mass. This sort of models may have various applications in physics of solid state and nano devices as well as in cosmology. The well developed SUSY approach to effective mass QM is rather promising for solution of different problems in physics. Some of suitable references are [206] - 212].

- The periodic potentials were studied actively in the framework of SUSY QM as well, see [213] - [222], [62] on one-dimensional models and [99] for two-dimensional quasi-exactly-solvable models with periodic potentials. Certainly, spectral design of periodic potential systems is promising, especially, in two-dimensional QM related to new nanoscale materials like graphene.

- The embedding of shape invariant potentials and their eigenfunctions into so called Discrete Quantum Mechanics has been analyzed recently [223], 224].

- The generalizations of conventional SUSY QM to the models with complex potentials [93], [96], 225] - 227] provide an effective tool in study of non-Hermitian [228] - 233] and, especially, PT symmetric [234] - [239] Quantum Mechanics with real energy spectrum (see reviews [240, [241]). Spectral design of nonHermitian systems with non-diagonalizable Hamiltonians was investigated both in one-dimensional [242, 243, 244] and two-dimensional [245] cases. PT-symmetric periodic potentials with SUSY have been investigated in [246].

- The polynomial SUSY in two dimensions has already brought a number of examples of new type of irreducible SUSY with hidden symmetries of higher-order in derivatives [88], [89]. An interesting sample of nonlinear supersymmetry with holomorphic supercharges for a two-dimensional fermion in external magnetic field was studied in[115]. One may expect a variety of new types of irreducible SUSY for third-order (and higher-order) supercharges as well as new discoveries in two and three dimensions.

- The perspective direction for investigations is the interrelations between the inverse scattering problem and methods of SUSY QM [247] - 252].

- Hidden and exotic nonlinear supersymmetries have been found in some models as, for instance, in pure parabosonic systems [132] and kink-antikink cristal [254]. There are also some connections of nonlinear SUSY with Conformal SUSY mechanics [255]. 
- The SUSY QM approach happens to be applicable and useful for building of different brane-world models with extra space dimension [256] - 259].

\section{Acknowledgments}

Many results presented in this review were obtained together with our colleagues Nikolay V. Borisov, Francesco Cannata, Jean-Pierre Dedonder, Michael I. Eides, Sengul Kuru, Juan Mateos Guilarte, Georg Junker, Javier Negro, Luismi Nieto, David N. Nishnianidze, Vyacheslav P. Spiridonov. We are grateful to all of them for very fruitful collaboration. We thank also our former and present students Andrey V. Sokolov, Alexey I. Neelov $\mathbb{q}$, Pavel A. Valinevich, Ekaterina V. Krupitskaya. A.A. acknowledges the partial financial support from projects FPA2010-20807, 2009SGR502, CPAN (Consolider CSD2007-00042). M.V.I. is grateful to Dr. V.V.Grigorian, whose skills have enabled him to complete successfully this work.

\section{References}

[1] Gendenshtein L E and Krive I V 1985 Supersymmetry in Quantum Mechanics Sov. Phys. Usp. 28645

[2] Kostelecky V A and Campbell D K 1985 Supersymmetry in physics. Proceedings: Conference, Los Alamos, USA, Dec 15-20, 1983 Physica 15D 1

[3] Lahiri A, Roy P K and Bagchi B 1990 Supersymmetry In Quantum Mechanics Int. J. Mod. Phys. A5 1383

[4] Cooper F, Khare A and Sukhatme U 1995 Supersymmetry and Quantum Mechanics Phys. Rept. 251267

[5] Junker G 1996 Supersymmetric methods in Quantum and Statistical Physics (Berlin: Springer)

[6] Bagrov V G and Samsonov B F 1997 Darboux transformation of the Schrodinger equation Phys. Part. Nucl. 28374

[7] Zakharev B N and Chabanov V M 1997 New situation in Quantum Mechanics (wonderful potentials from the inverse problem) Inv. Probl. $13 \mathrm{R} 47$

[8] Zakharev B N and Chabanov V M 1999 The Qualitative theory of elementary transformations of one and multichannel quantum systems in the inverse problem approach. The construction of transformations with given spectral parameters Phys. Part. Nucl. 30111

[9] Bagchi B 2001 Supersymmetry in Quantum and Classical Mechanics (Singapore: World Sci.)

[10] De Lima Rodrigues R 2002 The Quantum mechanics SUSY algebra: An introductory review Preprint hep-th/0205017

[11] Spiridonov V P 2003 The factorization method, self-similar potentials and quantum algebras In: Special Functions 2000: Current Perspective and Future Directions (Kluwer Acad. Publ.: Dordrecht) 335; Preprint hep-th/0302046

[12] Mielnik B and Rosas-Ortiz O 2004 Factorization: Little or great algorithm? J. Phys. A 3710007

[13] Ioffe M V 2004 SUSY-approach for investigation of two-dimensional quantum mechanical systems J. Phys. A 3710363

[14] Andrianov A A and Cannata F 2004 Nonlinear supersymmetry for spectral design in quantum mechanics J. Phys. A 3710297 hep-th/0407077

[15] Dong Shi-Hai 2007 Factorization Method in Quantum Mechanics (Dordrecht: Springer)

I Deceased. 
[16] Ioffe M V 2010 Supersymmetric separation of variables in two-dimensional Quantum Mechanics SIGMA 6075

[17] Gangopadhyaya A, Mallow J V and Rasinariu C 2011 Supersymmetric Quantum Mechanics: an introduction (Singapore: World Scientific)

[18] Ghosh P K 2012 Supersymmetric many-particle quantum systems with inverse-square interactions J. Phys. A 45183001

[19] Darboux G 1882 Sur une proposition relative aux équations linéaires $C$. R. Acad. Sci., Paris 94 1456; Darboux G 1999 Preprint physics/9908003 (in French)

[20] Moutard Th F 1875 C. R. Acad. Sci. Paris 80729

Moutard Th F 1879 J. de L'Ecole Politech. Chier 451

[21] Berkovich L M 2002 Factorization and transformations of differential equations: methods and applications (Sci.Publ.Center "Regular and Chaotic Dynamics", Moscow)[in Russian].

[22] Crum M M 1955 Associated SturmLiouville systems Quart. J. Math., Oxford 2, N6 121; Crum M M 1999 Preprint physics/9908019

[23] Euleri Leonhardi 1794 Methodus nova investigandi omnes casus quibus hans aequationem differentio-differentialen $\partial \partial y(1-a x x) b x \partial x \partial y-c y \partial x^{2}=0$. it M.S. Academiae exhibit aie 13 Ianuarii 1780 Institutiones calculi integralis 4533

[24] Delsart J and Lions J L 1957 Transmutations dopeŕateurs diffeŕentiels dans le domaine complexe Comment. Math. Helv. 32113 (in French)

[25] Krein M G 1957 On a continual analogue of a Christoffel formula from the theory of orthogonal polynomials Dokl. Akad. Nauk SSSR 113970 (in Russian)

[26] Faddeev L D 1963 The inverse problem in the quantum theory of scattering J. Math. Phys. 472 [1959 Usp. Mat. Nauk 14 57]

[27] Deift P A 1978 Applications of a commutation formula Duke Math. J. 45267

[28] Matveev V B and Salle M 1991 Darboux transformations and solitons (Berlin: Springer)

[29] Doktorov S V and Leble S B 2007 Dressing Method in Mathematical Physics (Dordrecht: Springer)

[30] Witten E 1981 Dynamical breaking of supersymmetry Nucl. Phys. B188 513

Witten E 1982 Constraints on supersymmetry breaking Nucl. Phys. B202 253

[31] Nicolai H 1976 Supersymmetry and spin systems J. Phys. A 91497

[32] Salomonson P and van Holten J W 1982 Fermionic coordinates and supersymmetry in Quantum Mechanics Nucl. Phys. B196 509

[33] Cooper F and Freedman B 1983 Aspects of Supersymmetric Quantum Mechanics Ann. Phys., NY 146262

[34] Schrödinger E 1940 A method of determining quantum-mechanical eigenvalues and eigenfunctions Proc. Roy. Irish Acad. A46 9

[35] Infeld L and Hull T E 1951 The factorization method Rev. Mod. Phys. 2321

[36] Andrianov A A, Borisov N V and Ioffe M V 1984 Quantum systems with identical energy spectra JETP Lett. 3993

Andrianov A A, Borisov N V and Ioffe M V 1984 The factorization method and quantum systems with equivalent energy spectra Phys. Lett. A105 19

Andrianov A A, Borisov N V and Ioffe M V 1984 Factorization method and Darboux transformation for multidimensional Hamiltonians Theor. Math. Phys. 611078

[37] Sukumar C V 1985 Supersymmetry, factorization of the Schrodinger equation and a Hamiltonian hierarchy J. Phys. A 18 L57

Sukumar C V 1985 Supersymmetric Quantum Mechanics of one-dimensional systems J. Phys. A 182917

[38] Mielnik B 1984 Factorization method and new potentials with the oscillator spectrum $J$. Math. Phys. 253387

[39] Fernández D J C 1984 New hydrogen - like potentials Lett. Math. Phys. 8337

(Fernández D J C 2000 Preprint physics/0006119)

[40] Akulov V P and Pashnev A I 1985 Supersymmetric quantum mechanics and spontaneous breaking 
of supersymmetry at the quantum level Theor. Math. Phys. 651027

[41] Imbo T D and Sukhatme U P 1985 Supersymmetric Quantum Mechanics and Large-N Expansions Phys. Rev. Lett. 542184

[42] Jarvis P D and Stedman G E 1986 Supersymmetry in second-order relativistic equations for the hydrogen atom J. Phys. A 191373

[43] Berezovoi V N and Pashnev A I 1987 Supersymmetric Quantum Mechanics And Rearrangement Of The Spectra Of Hamiltonians Theor. Math. Phys. 70102

[44] Riccati J F 1724 Animadversationes in aequationes differentiales secundi gradus Acta Erud. Suppl. 866

[45] Gendenshtein L E 1983 Derivation of exact spectra of the Schrodinger equation by means of supersymmetry JETP Lett. 38356

[46] Andrianov A A, Ioffe M V and Spiridonov V P 1993 Higher derivative supersymmetry and the Witten index Phys. Lett. A $\mathbf{1 7 4} 273$

[47] Andrianov A A, Cannata F, Dedonder J-P and Ioffe M V 1995 Second order derivative supersymmetry, Q deformations and scattering problem Int. J. Mod. Phys. A 10 2683; Preprint hep-th/9404061)

[48] Bagrov V G and Samsonov B F 1995 Darboux transformation of the Schrodinger equation Theor. Math. Phys. 1041051

[49] Spiridonov V 1996 Universal superpositions of coherent states and selfsimilar potentials Phys. Rev. A $\mathbf{5 2} 1909$

[50] Andrianov A A, Cannata F and Ioffe M V 1996 Contraction of higher order derivative SUSY algebras in Quantum Mechanics with large energy gap Mod. Phys. Lett. A 111417

[51] Fernández D J C 1997 SUSUSY Quantum Mechanics Int. J. Mod. Phys. A 12171 quant-ph/9609009.

[52] Fernández D J C, Hussin V and Mielnik B 1998 A Simple generation of exactly solvable anharmonic oscillators Phys. Lett. A 244309

[53] Akulov V P and Duplij S 1998 Nilpotent marsh and SUSY QM In: Dubna 1997, Supersymmetries and quantum symmetries 235; Preprint hep-th/9809089

[54] Samsonov B F 1996 New features in supersymmetry breakdown in Quantum Mechanics Mod. Phys. Lett. A 111563

Samsonov B F 1999 New possibilities for supersymmetry breakdown in Quantum Mechanics and second order irreducible Darboux transformations Phys. Lett. A 263274

[55] Junker J and Roy P 1998 Supersymmetric construction of exactly solvable potentials and nonlinear algebras Phys. Atom. Nucl. 611736

[56] Bagchi B and Ganguly A 1998 Generating isospectral Hamiltonians from a modified CrumDarboux transformation Int. J. Mod. Phys. A 133711

[57] Rosas-Ortiz J O 1998 New families of isospectral hydrogen-like potentials J. Phys. A 31 L507

Rosas-Ortiz J O 1998 Exactly solvable hydrogen-like potentials and factorization method J. Phys. A 3110163

[58] Plyushchay M S 1996 Deformed Heisenberg algebra, fractional spin fields and supersymmetry without fermions Ann. Phys. N.Y. 245339

[59] Fernández D J C and Hussin V 1999 Higher-order SUSY, linearized nonlinear Heisenberg algebras and coherent states J. Phys. A 323603

[60] Bagchi B, Ganguly A, Bhaumik D and Mitra A 1999 Higher derivative supersymmetry, a modified Crum-Darboux transformation and coherent state Mod. Phys. Lett. A 1427

[61] Mielnik B, Nieto L M and Rosas-Ortiz O 2000 The finite difference algorithm for higher order supersymmetries Phys. Lett. A 26970

[62] Fernández D J C, Negro J and Nieto L M 2000 Second-order supersymmetric periodic potentials Phys. Lett. A 275338

[63] Cariñena J F and Ramos A 2000 The partnership of potentials in Quantum Mechanics and shape invariance Mod. Phys. Lett. A 151079 
[64] Cariñena J F, Fernández D J C and Ramos A 2001 Group theoretical approach to the intertwined Hamiltonians Ann. Phys. 29242

[65] Aoyama A, Sato M and Tanaka T 2001 General forms of a N fold supersymmetric family Phys. Lett. B 503423

Aoyama A, Sato M and Tanaka T $2001 \mathrm{~N}$ fold supersymmetry in quantum mechanics: General formalism Nucl. Phys. B 619105

[66] Correa F, Plyushchay M S 2007 Hidden supersymmetry in quantum bosonic systems Ann. Phys. NY 3222493

[67] Tanaka T 2011 On a general form of $\mathcal{N}$-fold supersymmetry J. Phys. A 44465301

[68] Andrianov A A, Borisov N V, Eides M I and Ioffe M V 1984 Supersymmetric mechanics: a new look at the equivalence of quantum systems Theor. Math. Phys. 61965

Andrianov A A, Borisov N V, Eides M I and Ioffe M V 1985 Supersymmetric origin of equivalent quantum systems Phys. Lett. A 109143

[69] Andrianov A A and Ioffe M V 1988 Pauli fermions as components of N=2 Supersymmetric Quantum Mechanics Phys. Lett. B 205507

[70] Ioffe M V and Neelov A I 2003 Pauli equation and the method of supersymmetric factorization. J. Phys. A 362493

[71] Cannata F, Ioffe M V, Neelov A I and Nishnianidze D N 2004 Higher order matrix SUSY transformations in two-dimensional Quantum Mechanics J. Phys. A 3710339

[72] Andrianov A A, Borisov N V and Ioffe M V 1986 Nonstationary approach to scattering theory for supersymmetric Hamiltonians in Quantum Mechanics and supersymmetry Of nuclear interactions Phys. Lett. B 181141

Andrianov A A, Borisov N V and Ioffe M V 1986 Scattering theory for supersymmetric Hamiltonian and supersymmetry of nuclear interactions Theor. Math. Phys. 72748

[73] Andrianov A A and Sokolov A V 2003 Nonlinear supersymmetry in Quantum Mechanics: algebraic properties and differential representation Nucl. Phys. B 66025

[74] Bagrov V G and Samsonov B F 2002 On irreducible second-order Darboux transformations Russ. Phys. J. 4527

[75] Andrianov A A and Sokolov A V 2007 Factorization of Nonlinear Supersymmetry in Onedimensional Quantum Mechanics. I. General classification of reducibility and analysis of the third-order algebra J. Math. Sci. 1432707

[76] Sokolov A V 2007 Factorization of Nonlinear Supersymmetry in One-dimensional Quantum Mechanics. II. Proofs of theorems on reducibility J. Math. Sci. 1432924

[77] Sokolov A V 2010 Factorization of Nonlinear Supersymmetry in One-dimensional Quantum Mechanics. III. Precise classification of irreducible intertwining operators J. Math. Sci. 168 881

[78] Andrianov A A, Cannata F, Ioffe M V and Nishnianidze D N 2000 Systems with higher order shape invariance: Spectral and algebraic properties Phys. Lett. A 266341 (Preprint quant-ph/9902057)

[79] E. L. Ince 1956 Ordinary Differential Equations (Dover Publications, Inc., New York)

Painleve Transcendents, Their Asymptotics and Physical Applications 1992 Eds. P. Winternitz and D. Levi, NATO ASI Series B, (Plenum Press, New York)

[80] Ioffe M V and Nishnianidze D N 2004 SUSY intertwining relations of third order in derivatives Phys. Lett. A 327425

[81] Bermúdez D and Ferández D J C 2011 Supersymmetric Quantum Mechanics and Painlevé IV Equation SIGMA 7025

[82] Marquette J 2009 Superintegrability with third order integrals of motion, cubic algebras and supersymmetric quantum mechanics II: Painlevé transcendent potentials J. Math. Phys. 50 095202

[83] Fernández D J C and Hussin V 2001 Polynomial Heisenberg algebras and higher order supersymmetry Preprint quant-ph/0011004 
[84] Willox R and Hietarinta J 2003 Painlevé equations from Darboux chains: I. $P_{I I I} P_{V}$ J. Phys. A 3610615

[85] Carballo J M, Fernández D J C, Negro J and Nieto L M 2004 Polynomial Heisenberg algebras J. Phys. A 3710349

[86] Bermudez D and Fernandez D J C 2011 Non-hermitian Hamiltonians and Painlevé IV equation with real parameters Phys. Lett. A 3752974

[87] Cannata F, Ioffe M, Junker G and Nishnianidze D 1999 Intertwining relations of nonstationary Schrodinger operators J. Phys. A 323583

[88] Andrianov A A, Ioffe M V and Nishnianidze D N 1995 Polynomial SUSY in Quantum Mechanics and second derivative Darboux transformation Phys. Lett. A 201103

[89] Andrianov A A, Ioffe M V and Nishnianidze D N 1995 Polynomial supersymmetry and dynamical symmatries in Quantum Mechanics Theor. Math. Phys. 1041129

[90] Andrianov A A, Ioffe M V and Nishnianidze D N 1996 Higher order SUSY in Quantum Mechanics and integrability of two-dimensional Hamiltonians, Preprint: solv-int/9605007

[91] Andrianov A A, Ioffe M V and Nishnianidze D N 1999 Classical integrable 2-dim models inspired by SUSY Quantum Mechanics J. Phys. A 324641

[92] Cannata F, Ioffe M V and Nishnianidze D N 2002 New methods for two-dimensional Schrodinger equation: SUSY-separation of variables and shape invariance J. Phys. A 351389

[93] Cannata F, Ioffe M V and Nishnianidze D N 2003 Two-dimensional SUSY-pseudo-Hermiticity without separation of variables Phys. Lett. A 310344

[94] Ioffe M V and Valinevich P A 2005 New two-dimensional quantum models partially solvable by supersymmetrical approach J. Phys. A 382497

[95] Cannata F, Ioffe M V and Nishnianidze D N 2005 Double shape invariance of two-dimensional singular Morse model Phys. Lett. A 34031

[96] Cannata F, Ioffe M V and Nishnianidze D N 2006 Exactly solvable two-dimensional complex model with real spectrum Theor. Math. Phys. 148960

[97] Ioffe M V, Mateos Guilarte J and Valinevich P A 2006 Two-dimensional supersymmetry: from SUSY Quantum Mechanics to integrable classical models Ann. Phys. 3212552

[98] Ioffe M V, Negro J, Nieto L M and Nishnianidze D N 2006 New two-dimensional integrable quantum models from SUSY intertwining J. Phys. A 399297

[99] Ioffe M V, Mateos Guilarte J and Valinevich P A 2008 A class of partially solvable two-dimensional quantum models with periodic potentials Nucl. Phys. B $\mathbf{7 9 0} 414$

[100] Ioffe M V and Nishnianidze D N 2007 Exact solvability of two-dimensional real singular Morse potential Phys. Rev. A $\mathbf{7 6} 052114$

[101] Cannata F, Ioffe M V and Nishnianidze D N 2009 Three-dimensional solutions of supersymmetrical intertwining relations and pairs of isospectral Hamiltonians J. Math. Phys. 50052105

[102] Ioffe M V, Nishnianidze D N and Valinevich P A 2010 New exactly solvable two-dimensional quantum model not amenable to separation of variables J. Phys. A 43485303

[103] Cannata F, Ioffe M V and Nishnianidze D N 2011 New two-dimensional quantum models with shape invariance J. Math. Phys. 52022106

[104] Ioffe M V, Krupitskaya E V and Nishnianidze D N 2012 Analytical solution of two-dimensional Scarf II model by means of SUSY methods Ann. Phys. 327764

[105] Ioffe M V, Krupitskaya E V and Nishnianidze D N 2012 Supersymmetrical separation of variables for Scarf II model: partial solvability Europhysics Letters $\mathbf{9 8} 10013$

[106] Klishevich S M and Plyushchay M S 2001 Nonlinear supersymmetry, quantum anomaly and quasiexactly solvable systems Nucl. Phys. B 606583

Klishevich S M and Plyushchay M S 2002 Nonlinear holomorphic supersymmetry, Dolan-Grady relations and Onsager algebra Nucl. Phys. B 628217

Klishevich S M and Plyushchay M S 2002 Nonlinear holomorphic supersymmetry on Riemann surfaces Nucl. Phys. B 640481 
[107] Demircioglu B, Kuru S, Onder M and Vercin A 2002 Two families of superintegrable and isospectral potentials in two dimensions. J. Math. Phys. 432133

Kuru S, Tegmen A and Vercin A 2001 Intertwined isospectral potentials in an arbitrary dimension. J. Math. Phys. 423344

[108] Freedman D Z and Mende P F 1990 An exactly solvable N particle system in Supersymmetric Quantum Mechanics Nucl. Phys. B 344317

[109] Kirchberg A, Lange J D, Pisani P A G and Wipf A 2003 Algebraic solution of the supersymmetric hydrogen atom in d-dimensions Ann. Phys. 303359

[110] De Crombrugghe M and Rittenberg V 1983 Supersymmetric Quantum Mechanics Ann. Phys. 15199

[111] Khare A and Maharana J 1984 Supersymmetric Quantum Mechanics in one-dimension, twodimensions and three-dimensions Nucl. Phys. B 244409

[112] Cooper F, Khare A, Musto R and Wipf A 1988 Supersymmetry and the Dirac equation Ann. Phys. 1871

[113] Gendenshtein L E 1984 Supersymmetry in the problem of an electron in a nonuniform magnetic field. JETP Lett. 39280

[114] Ioffe M V, Kuru S, Negro J and Nieto L M 2006 SUSY approach to Pauli Hamiltonians with an axial symmetry J. Phys. A 396987

[115] Klishevich S M, Plyushchay M S 2001 Nonlinear supersymmetry on the plane in magnetic field and quasi-exactly solvable systems Nucl.Phys. B $\mathbf{6 1 6} 403$

[116] Weinberg S 2000 The Quantum Theory of Fields. Vol. III. Supersymmetry (Cambridge Univ. Press);

[117] de Alfaro V, Fubini S, Furlan G and Rossetti C 1973 Currents in Hadron Physics (North Holland, Amsterdam)

[118] Borisov N V, Ioffe M V and Eides M I 1976 Second quantization of field systems on the Grassmann algebra: dual strings with internal spin Theor. Math. Phys. 29906

[119] Urrutia L F and Hernandez E 1983 Long range behavior of nuclear forces as a manifestation of supersymmetry in nature Phys. Rev. Lett. 51755

[120] Plyushchay M 2004 Nonlinear supersymmetry: From classical to quantum mechanics Preprint hep-th/0402025

[121] Fedoruk S, Ivanov E and Lechtenfeld O 2012 Superconformal Mechanics J. Phys. A 45173001

[122] Adler V E 1994 A modification of Crum's method Theor. Math. Phys. 1011381

[123] Shabat A B 1992 The infinite-dimensional dressing dynamical system Inv. Probl. 8303

[124] Andreev V, Kharchev S and Shmakova M 1993 Closed chains of supersymmetrical Hamiltonians Phys. Lett. A 181142

[125] Veselov A P and Shabat A B 1993 Dressing chains and the spectral theory of the Schrödinger operator Funct. Anal. Appl. 2781

[126] Smilga A V 2003 Weak supersymmetry Preprint hep-th/0311023

[127] Rubakov V A and Spiridonov V P 1988 Parasupersymmetric Quantum Mechanics Mod. Phys. Lett. A 31337

[128] Durand S and Vinet S 1989 Conformal parasupersymmetry in quantum mechanics Mod. Phys. Lett. A 42519

[129] Beckers J and Debergh N 1990 Parastatistics and supersymmetry in quantum mechanics Nucl. Phys. B 340767

[130] Andrianov A A and Ioffe M V 1991 From supersymmetric quantum mechanics to a parasupersymmetric one Phys. Lett. B 255543

[131] Andrianov A A, Ioffe M V, Spiridonov V P and Vinet L 1991 Parasupersymmetry and truncated supersymmetry in quantum mechanics Phys. Lett. B 272297

[132] Plyushchay M 2000 Hidden nonlinear supersymmetries in pure parabosonic systems Int. J. Mod. Phys. A 153679

[133] Fernández D J C, Munoz R and Ramos A 2003 Second order SUSY transformations with complex 
energies Phys. Lett. A 30811

[134] Debergh N, Van den Bossche B and Samsonov B F 2002 Darboux transformations for quasiexactly solvable Hamiltonians Int. J. Mod. Phys. A 171577

[135] Fernández D J C and Salinas-Hernández E 2003 The Confluent algorithm in second order supersymmetric Quantum Mechanics J. Phys. A 362537

[136] Naimark M A 1967 Linear differential operators ( Frederick Ungar Publishing Co., New York)

[137] Flügge S 1971 Practical Quantum Mechanics (Berlin-Heidelberg: Springer)

[138] Zakhariev B N and Suzko A A 1990 Direct and Inverse Methods (Springer, Berlin-Heidelberg: Springer)

[139] Fernández D J C, Mielnik B, Rosas-Ortiz O and Samsonov B F 2002 The phenomenon of Darboux displacements Phys. Lett. A 294168

[140] Burchnall J L and Chaundy T W 1923 Commutative ordinary differential operatorsProc. London Math. Soc. Ser. 221420

[141] Correa F, Jakubsky V and Plyushchay M S 2008 Finite-gap systems, tri-supersymmetry and self-isospectrality J.Phys. A 41485303

[142] Dabrowska J W, Khare A and Sukhatme U P 1988 Explicit wavefunctions for shape-invariant potentials by operator techniques J. Phys. A $21 \mathrm{~L} 195$

[143] Gangopadhyaya A, Mallow J V and Sukhatme U P 1998 Translational shape invariance and the inherent potential algebra Phys. Rev. A 584287

[144] Gangopadhyaya A and Mallow J V 2008 Generating shape invariant potentials Int. J. Mod. Phys. A 234959

[145] Bougie J, Gangopadhyaya A and Mallow J V 2010 Generation of a complete set of supersymmetric shape invariant potentials from an Euler equation Phys. Rev. Lett. 105210402

[146] Correa F, del Olmo M A and Plyushchay M S 2005 On hidden broken nonlinear superconformal symmetry of conformal mechanics and nature of double nonlinear superconformal symmetry Phys.Lett. B 628157

[147] Bagrov V G and Samsonov B F 1996 Supersymmetry of a nonstationary Schrödinger equation Phys. Lett. A 21060

[148] Finkel F, González-López A, Kamran N and Rodríguez M A 1999 On form-preserving transformations for the time-dependent Schro" dinger equation J. Math. Phys. 403268

[149] Beckers J, Debergh N and Gotti C 1998 On Generalized Darboux transformations and symmetries of Schrodinger equation Helv. Phys. Acta $\mathbf{7 1} 214$

[150] Boyer C P 1974 The maximal kinematical invariance group for an arbitrary potential Helv. Phys. Acta $\mathbf{4 7} 589$

[151] Fushchych W I and Nikitin A G 1997 Higher symmetries and exact solutions of linear and nonlinear Schro" dinger equation J. Math. Phys. 385944

[152] Bluman G and Shtelen V 1996 New classes of Schrödinger equations equivalent to the free particle equation through non-local transformations J. Phys. A 294473

[153] Miller Jr. W 1977 Symmetry and Separation of Variables (Addison-Wesley Publishing Company, London);

[154] Perelomov A M 1990 Integrable systems of Classical Mechanics and Lie Algebras (Birkhäuser,Boston, MA)

[155] Hietarinta J 1987 Direct methods for the search of the second invariant Phys. Rep. 14787

[156] Eisenhart L P 1948 Enumeration of potentials for which one-particle Schroedinger equations are separable Phys. Rev. $\mathbf{7 4} 87$

Eisenhart L P 1934 Separable systems of Stäckel Ann. Math. 35284

[157] Razavy M 1981 A potential model for torsional vibrations of molecules Phys. Lett. A 827

[158] Alhassid Y, Gursey F and Iachello F, 1983 Group theory approach to scattering Ann. Phys. 148 346

[159] Turbiner A V 1988 Quasi-exactly-solvable problems and sl(2) algebra Comm. Math. Phys. 118 467 
[160] Ushveridze A G 1994 Quasi-Exactly Solvable Problems in Quantum Mechanics (Bristol: Institute of Physics Publishing)

[161] Shifman M A, Turbiner A V 1989 Quantal problems with partial algebraization of the spectrum Comm. Math. Phys. 126347

[162] Shifman M A 1989 New findings In Quantum Mechanics (partial algebraization of the spectral problem) Int. J. Mod. Phys. A 42897

[163] Kamran N, Olver P J 1990 Lie algebras of differential operators and Lie-algebraic potentials $J$. Math. Anal. Appl. 145342

[164] Shifman M A 1989 Supersymmetric Quantum Mechanics and partial algebraization of the spectral problem Int. J. Mod. Phys. A 43305

[165] Calogero F 1971 Solution of the one-dimensional N body problems with quadratic and/or inversely quadratic pair potentials J. Math. Phys. 12419

[166] Tremblay F, Turbiner A V and Winternitz P 2009 An infinite family of solvable and integrable quantum systems on a plane J. Phys. A 42242001

Tremblay F, Turbiner A V and Winternitz P 2010 Periodic orbits for an infinite family of classical superintegrable systems J. Phys. A 43015202

[167] Quesne C $2010 \mathrm{~N}=2$ supersymmetric extension of the TremblayTurbinerWinternitz Hamiltonians on a plane J. Phys. A $\mathbf{4 3} 305202$

Quesne C 2010 Superintegrability of the TremblayTurbinerWinternitz quantum Hamiltonians on a plane for odd k J. Phys. A 43082001

[168] Marquette J 2009 Supersymmetry as a method of obtaining new superintegrable systems with higher order integrals of motion J. Math. Phys. 50122102

Marquette J 2011 An infinite family of superintegrable systems from higher order ladder operators and supersymmetry J. Phys. Conf. Ser. 284012047

[169] Landau L D and Lifshitz E M 1965 Quantum Mechanics (Non-relativistic Theory) (Pergamon Press, 2nd edition, London) Sect. 23 (Problem 3)

[170] Minahan J A and Polychronacos A P 1993 Integrable systems for particles with internal degrees of freedom Phys. Lett. B $\mathbf{3 0 2} 265$

[171] Efthimiou C and Spector D 1997 Shape invariance in the Calogero and Calogero-Sutherland models Phys. Rev. A 56208

[172] Brink L, Turbiner A V and Wyllard N 1998 Hidden algebras of the (super)Calogero and Sutherland models J. Math. Phys. 391285

[173] Ioffe M V and Neelov A I 2000 Multiparticle SUSY Quantum Mechanics and the representations of permutation group J. Phys. A 331581

[174] Bordner A J, Manton N S and Sasaki R 2000 Calogero-Moser models V: Supersymmetry and quantum Lax pair Prog. Theor. Phys. 103463

[175] Cannata F and Ioffe M V 2001 Exactly solvable three-body SUSY systems with internal degrees of freedom J. Phys. A 341129

[176] Sasaki R, Takasaki K 2001 Quantum Inozemtsev model, quasi-exact solvability and N-fold supersymmetry J. Phys. A 34 9533; Erratum - ibid 3410335

[177] Ioffe M V and Neelov A I 2002 Intertwining relations for the matrix Calogero-like models: supersymmetry and shape invariance J. Phys. A 357613

[178] Andrianov A A, Cannata F, Ioffe M V and Nishnianidze D N 1997 Matrix Hamiltonians: SUSY approach to hidden symmetries J. Phys. A 305037

[179] De Lima Rodrigues R and Vaidya A N 2003 SUSY QM via 2x2 matrix superpotential Preprint hep-th/0308189

[180] Samsonov B F and Pecheritsin A A 2004 Chains of Darboux transformations for the matrix Schrödinger equation J. Phys.A 37239

[181] Nikitin A G and Karadzhov Y 2011 Enhanced classification of matrix superpotentials J. Phys. A 44445202

[182] Nikitin A G and Karadzhov Y 2011 Matrix superpotentials J. Phys. A 44305204 
[183] Karadzhov Y 2012 Matrix superpotential linear in variable parameter Comm. Nonlin. Sci. Numer. Simulat. 171522

[184] Tanaka T 2012 N-fold supersymmetry in quantum mechanical matrix models Mod. Phys. Lett. A 271250051

[185] Cooper F, Ginocchio J N and Wipf A 1988 Derivation of the S matrix using supersymmetry Phys. Lett. A 129145

[186] Amado R D , Cannata F and Dedonder J-P 1988 Coupled channel Supersymmetric Quantum Mechanics Phys. Rev. A 383797

Amado R D , Cannata F and Dedonder J-P 1990 Supersymmetric Quantum Mechanics, coupled channels, scattering relations Int. J. Mod. Phys. A 53401

[187] Fukui T 1993 Shape-invariant potentials for systems with multi-component wave functions Phys. Lett. A 1781

[188] Correa F, Falomir H, Jakubsky V and Plyushchay M S 2010 Supersymmetries of the spin-1/2 particle in the field of magnetic vortex, and anyons Ann. Phys. 3252653

[189] Nikitin A G 2012 Matrix superpotentials and superintegrable systems for arbitrary spin J. Phys. A: Math. Theor. 45225205

[190] Jakubsky V and Plyushchay M S 2012 Supersymmetric twisting of carbon nanotubes Phys. Rev. D 85045035

[191] Cannata F and Ioffe M V 1992 Solvable coupled channel problems from supersymmetric quantum mechanics Phys. Lett. B $\mathbf{2 7 8} 399$

Cannata F and Ioffe M V 1993 Coupled-channel scattering and separation of coupled differential equations by generalized Darboux transformations J. Phys. A 26 L89

[192] Das T K and Chakrabarti B 1999 Application of supersymmetry to a coupled system of equations: the concept of a superpotential matrix J. Phys. A 322387

[193] Sparenberg J-M, Samsonov B, Foucart F and Baye D 2006 Multichannel coupling with supersymmetric quantum mechanics and exactly-sovable model for Feshbach resonance. $J$. Phys. A 39 L639

[194] Pupasov A M, Samsonov B F, Sparenberg J-M and Baye D 2009 Coupling between scattering channels with SUSY transformations for equal thresholds J. Phys. A 42195303

[195] Cannata F, Ioffe M V, Neelov A I and Nishnianidze D N 2004 Higher order matrix SUSY transformations in two-dimensional Quantum Mechanics J. Phys. A 3710339

[196] Correa F, Dunne G V and Plyushchay M S 2009 The Bogoliubov-de Gennes system, the AKNS hierarchy, and nonlinear quantum mechanical supersymmetry Annals Phys. 3242522

[197] Samsonov B F 2004 Darboux transformations of coherent states of the time-dependent singular oscillator J. Phys. A $\mathbf{3 7} 10273$

[198] Samsonov B 1997 Supersymmetry and supercoherent states of a nonrelativistic free particle $J$. Math. Phys. 384492

[199] Samsonov B 2000 Coherent states for transparent potentials J. Phys. A 33591

[200] Fernandez D J, Hussin V and Rosas-Ortiz O 2007 Coherent states for Hamiltonians generated by supersymmetry J. Phys. A 406491

[201] Angelova M and Hussin V 2008 Generalized and Gaussian coherent states for the Morse potential J. Phys. A 4130416

[202] Andrianov A A, Ioffe M V and Tsu Zhun-Pin 1988 Factorization method in curvilinear coordinates and pairing of levels for matrix potentials Vestnik Leningradskogo Universiteta. Ser.4 Fiz.Khim. 43 (in Russian); [English translation - Preprint:1101.0773]

[203] Gonzalez Leon M A, Mateos Guilarte J and de la Torre M 2007 Two-dimensional Supersymmetric Quantum Mechanics: two fixed centers of force SIGMA 3124

[204] Gonzalez Leon M A, Mateos Guilarte J, de la Torre Mayado M 2012 Factorization of supersymmetric Hamiltonians in curvilinear coordinates J. Phys.: Conf. Ser. 343012040

[205] Samani K A and Zarei M 2005 Intertwined Hamiltonians in two-dimensional curved spaces Ann. Phys. 316466 
[206] Bagchi B, Gorain P, Quesne C and Roychoudhury R 2004 Effective-mass Schroedinger equation and generation of solvable potentials Czech. J. Phys. 541019

[207] Bagchi B, Gorain P, Quesne C and Roychoudhury R 2005 New approach to (quasi)-exactly solvable Schrodinger equations with a position-dependent effective mass Europhys. Lett. $\mathbf{7 2}$ 155

[208] Bagchi B, Banerjee A, Quesne C and Tkachuk V M 2005 Deformed shape invariance and exactly solvable Hamiltonians with position-dependent effective mass J. Phys. A 382929

[209] Quesne C 2009 Point canonical transformation versus deformed shape invariance for positiondependent mass Schrodinger equations SIGMA 5046

[210] Ganguly A, Ioffe M V and Nieto L M 2006 A new effective mass Hamiltonian and associated Lame equation: bound states J. Phys. A 3914659

[211] Cannata F, Ioffe M V and Nishnianidze D N 2008 Two-dimensional Schrodinger Hamiltonians with effective mass in SUSY approach Ann. Phys. 3232624

[212] Midya B, Roy B and Tanaka T 2012 Effect of Position-dependent Mass on Dynamical Breaking of Type B and Type $X_{2} N$-fold Supersymmetry J. Phys. A 45205303

[213] Braden H W and Macfarlane A J 1985 Supersymmetric Quantum Mechanical Models With Continuous Spectrum And The Witten Index, J. Phys. A 183151

[214] Dunne G and Feinberg J 1998 Self-isospectral periodic potentials and Supersymmetric Quantum Mechanics Phys. Rev. D 571271

[215] Khare A and Sukhatme U 1999 New solvable and quasi exactly solvable periodic potentials $J$. Math. Phys. 405473

Khare A and Sukhatme U 2001 Some exact results for mid-band and zero band-gap states of associated Lame potentials J. Math. Phys. 425652

Khare A and Sukhatme U 2004 Periodic potentials and supersymmetry J. Phys. A 3710037

[216] Dunne G and Shifman M 2002 Duality and self-duality (energy reflection symmetry) of quasiexactly solvable periodic potentials Ann. Phys. 299143

[217] Fernandez C D J, Mielnik B, Rosas-Ortiz O and Samsonov B F 2002 Nonlocal SUSY deformations of periodic potentials J. Phys. A 354279

[218] Samsonov B F, Glasser M L, Negro J and Nieto L M 2003 Second-order Darboux displacements J. Phys. A 3610053

[219] Fernandez C D J and Ganguly A 2007 Exactly solvable associated Lame potentials and supersymmetric transformations Ann. Phys. 3221143

[220] Correa F, Nieto L M and Plyushchay M 2007 Hidden nonlinear supersymmetry of finite-gap Lame equation Phys.Lett. B 64494

[221] Andrianov A A and Sokolov A V 2009 Hidden Symmetry from Supersymmetry in OneDimensional Quantum Mechanics SIGMA 5064

[222] Correa F, Jakubsky V, Nieto L-M, Plyushchay M S 2008 Self-isospectrality, special supersymmetry, and their effect on the band structure Phys.Rev.Lett. 101030403

[223] Spiridonov V, Vinet L and Zhedanov A 1993 Difference Schroedinger operators with linear and exponential discrete spectra Lett. Math. Phys. 2963

[224] Odake S and Sasaki R 2005 Shape invariant potentials in discrete Quantum Mechanics J. Nonlin. Math. Phys. 12 Suppl. 1507

Odake S and Sasaki R 2005 Equilibrium positions, shape invariance and Askey-Wilson polynomials J. Math. Phys. 46063513

Odake S and Sasaki R 2009 Exactly solvable discrete quantum mechanics: shape invariance, Heisenberg solutions, annihilation-creation operators and coherent states Prog. Theor. Phys. 119663

Odake S and Sasaki R 2011 Discrete Quantum Mechanics J. Phys. A 44353001

[225] Andrianov A A, Cannata F, Ioffe M V and Dedonder J P 1999 SUSY Quantum Mechanics with complex superpotentials and real energy spectra Int. J. Mod. Phys. A 142675

[226] Cannata F, Junker G and Trost J 1998 Schrodinger operators with complex potential but real 
spectrum Phys. Lett. A 246219

[227] Cannata F, Ioffe M V and Nishnianidze D N 2007 Pseudo-Hermiticity of an Exactly Solvable Two-Dimensional Model Phys. Lett. A 3699

[228] Berry M V 2004 Physics of non-Hermitian degeneracies Czechoslovak J. Phys. 541039

[229] Heiss W D 2004 Exceptional points of non-Hermitian operators J. Phys. A 372455

[230] Curtright T and Mezincescu L 2007 Biorthogonal quantum systems J. Math. Phys. 48092106

[231] Müller M and Rotter I 2008 Exceptional points in open quantum systems J. Phys. A 41244018

[232] Bagarello F 2010 Mathematical aspects of intertwining operators: the role of Riesz bases J. Phys. A 43175203

[233] Olkhovsky V S, Maydanyuk S P and Recami E 2010 Non-self-adjoint operators as observables in quantum theory and nuclear physics Phys. Part. Nucl. 41508

[234] Bender C M, Boettcher S and Meisinger P N 1999 PT-symmetric Quantum Mechanics J. Math. Phys. 402201

[235] Bender C M, Brody D C and Jones H F 2002 Complex extension of Quantum Mechanics Phys. Rev. Lett. 89270401

[236] Dorey P, Dunning C and Tateo R 2001 Spectral equivalences, Bethe Ansatz equations, and reality properties in PT-symmetric Quantum Mechanics J. Phys. A 345679

[237] Mostafazadeh A 2002 Pseudosupersymmetric Quantum Mechanics and isospectral pseudoHermitian Hamiltonians Nucl. Phys. B $\mathbf{6 4 0} 419$

Mostafazadeh A 2002 Pseudo-Hermiticity for a class of nondiagonalizable Hamiltonians J. Math. Phys. 436343

[238] Ahmed Z 2002 Pseudo-Hermiticity of Hamiltonians under gauge-like transformation: real spectrum of non-Hermitian Hamiltonians Phys. Lett. A 294287

[239] Japaridze G S 2002 Space of state vectors in PT-symmetric Quantum Mechanics J. Phys. A 35 1709

[240] Bender C M 2005 Introduction to PT-symmetric quantum theory Contemp. Phys. 46277

Bender C M 2007 Making sense of non-Hermitian Hamiltonians Rept. Prog. Phys. 70947

[241] Mostafazadeh A and Batal A 2004 Physical aspects of pseudo-Hermitian and PT-symmetric Quantum Mechanics J. Phys. A 3711645

Mostafazadeh A 2010 Pseudo-Hermitian representation of Quantum Mechanics Int. J. Geom. Meth. Mod. Phys. 71191

[242] Sokolov A V, Andrianov A A and Cannata F 2006 Non-Hermitian quantum mechanics of nondiagonalizable Hamiltonians: puzzles with self-orthogonal states J. Phys. A 3910207

Andrianov A A, Cannata F and Sokolov A V 2007 Non-linear supersymmetry for non-Hermitian, non-diagonalizable Hamiltonians. I. General properties Nucl. Phys. B $\mathbf{7 7 3} 107$

Andrianov A A, Cannata F and Sokolov A V 2010 Spectral singularities for non-Hermitian onedimensional Hamiltonians: puzzles with resolution of identity J. Math. Phys. 51052104

[243] Andrianov A A and Sokolov A V 2011 Resolutions of Identity for Some Non-Hermitian Hamiltonians. I. Exceptional Point in Continuous Spectrum SIGMA 7111

[244] Sokolov A V 2007 Non-linear supersymmetry for non-Hermitian, non-diagonalizable Hamiltonians. II. Rigorous results Nucl. Phys. B $\mathbf{7 7 3} 137$

Sokolov A V 2011 Resolutions of identity for some non-Hermitian Hamiltonians. II. Proofs SIGMA 7112

[245] Cannata F, Ioffe M V and Nishnianidze D N 2010 Exactly solvable non-separable and nondiagonalizable 2-dim model with quadratic complex interaction J. Math. Phys. 51022108

Cannata F, Ioffe M V and Nishnianidze D N 2012 Equidistance of the complex 2-dim anharmonic oscillator spectrum: exact solution J. Phys. A 45295303

[246] Correa F, Plyushchay M S 2012 Self-isospectral tri-supersymmetry in PT-symmetric quantum systems with pure imaginary periodicity Annals Phys. 3271761

[247] Nieto M M 1984 Relationship between supersymmetry and the inverse method in Quantum Mechanics Phys. Lett. B 145208 
[248] Sukumar C V 1985 Supersymmetric Quantum Mechanics and the inverse scattering method $J$. Phys. A 182937

[249] Sukumar C V 1986 Supersymmetry, potentials with bound states at arbitrary energies and multi - soliton configurations J. Phys. A 192297

[250] Kwong W and Rosner J L 1986 Supersymmetric Quantum Mechanics and inverse scattering Prog. Theor. Phys. Suppl. 86366

[251] Baye D 1987 Supersymmetry between deep and shallow nucleus-nucleus potentials Phys. Rev. Lett. $\mathbf{5 8} 2738$

[252] Sparenberg J M and Baye D 2004 Inverse scattering with Supersymmetric Quantum Mechanics J. Phys. A 3710223

[253] Jakubsky V, Nieto L-M and Plyushchay M S 2010 The origin of the hidden supersymmetry Phys.Lett. B 69251

[254] Plyushchay M S and Nieto L-M 2010 Self-isospectrality, mirror symmetry, and exotic nonlinear supersymmetry Phys.Rev. D $\mathbf{8 2} 065022$

Plyushchay M S, Arancibia A and Nieto L-M 2011 Exotic supersymmetry of the kink-antikink cristal, and infinite period limit Phys.Rev. D $\mathbf{8 3} 065025$

[255] Leiva C and Plyushchay M S 2003 Superconformal mechanics and nonlinear supersymmetry $J$. High Energy Phys. 10069

[256] Andrianov A A, Andrianov V A, Giacconi P and Soldati R 2003 Domain wall generation by fermion self-interaction and light particles JHEP 07063 (Appendix B)

[257] Lim C S, Nagasawa T, Ohya S, Sakamoto K and Sakamoto M 2008 Supersymmetry in 5d gravity Phys. Rev. D $\mathbf{7 7} 045020$

[258] Nagasawa T, Ohya S, Sakamoto K, Sakamoto M and Sekiya K 2009 Hierarchy of QM SUSYs on a bounded domain J. Phys. A 42265203

[259] Nagasawa T, Ohya S, Sakamoto K and Sakamoto M 2011 Emergent supersymmetry in warped backgrounds SIGMA 7065 